Version 6.6c, 1 June 2019: Final clean-up.

\title{
Occultation observations of Saturn's rings with Cassini VIMS
}

\author{
Philip D. Nicholson*, Todd Ansty \\ Department of Astronomy, Cornell University, Ithaca NY 14853 \\ Matthew M. Hedman, Douglas Creel, Johnathon Ahlers, \\ Department of Physics, University of Idaho, Moscow ID 83844 \\ Rebecca A. Harbison \\ Department of Physics, University of Nebraska, Lincoln NE 68588
}

Robert H. Brown

Lunar $\&$ Planetary Laboratory, University of Arizona, Tucson AZ 85721

Roger. N. Clark

Planetary Science Institute, 1700 East Fort Lowell, Suite 106, Tucson AZ 85719

Kevin H. Baines, Bonnie J. Buratti, Christophe Sotin

Jet Propulsion Laboratory, Pasadena CA 91109

Sarah V. Badman

Department of Physics, Lancaster University, Lancaster LA1 4YB, United Kingdom.

* Corresponding author, nicholso@astro.cornell.edu

\begin{abstract}
We describe the prediction, design, execution and calibration of stellar and solar occultation observations of Saturn's rings by the Visual and Infrared Mapping Spectrometer (VIMS) instrument on the Cassini spacecraft. Particular attention is paid to the technique developed for onboard acquisition of the stellar target and to the geometric and photometric calibration of the data. Examples of both stellar and solar occultation data are presented, highlighting several aspects of the data as well as the different occultation geometries encountered during Cassini's 13 year orbital tour. Complete catalogs of ring stellar and solar occultations observed by Cassini-VIMS are presented, as a guide to the standard data sets which have been delivered to the Planetary Data System's Ring Moon Systems Node (Hedman and Nicholson 2019b).
\end{abstract}

Subject Keywords: occultations; Saturn, rings; dynamics 


\section{Introduction}

Detailed studies of the structure and dynamics of Saturn's rings were among the primary scientific goals of the Cassini-Huygens mission (Matson et al. 2004). In addition to the many thousands of images taken by the spacecraft's Imaging Science Subsystem (ISS), the principal sources of data for such studies are occultation experiments carried out at multiple wavelengths (Colwell et al. 2009). Each such observation provided a single radial profile of the ring's transmission at a particular time and longitude, and a specific value of the ring opening angle $B$. Cassini's science payload included four instruments capable of carrying out occultation observations, two of which were designed with this purpose in mind.

The Radio Science Subsystem (RSS) used the spacecraft communication system to obtain simultaneous occultation data at wavelengths of 1.3, 3.5 and $12.6 \mathrm{~cm}$ (Ka, X and Sbands, respectively) (Kliore et al. 2004). Although the raw RSS data are limited by Fresnel diffraction to a radial resolution of a few kilometers, the coherent nature of the transmitted signal makes it possible to 'invert' the data to obtain diffraction-corrected profiles with resolutions of $400 \mathrm{~m}$ or better, subject to limitations imposed by SNR considerations (Marouf et al. 1986, 2007, 2010).

The High Speed Photometer channel of the Ultraviolet Imaging Spectrometer (UVIS) observed early-type stars through the rings at a wavelength of $150 \mathrm{~nm}$, obtaining radial optical depth profiles with sampling intervals as short as $1 \mathrm{msec}$, corresponding to a nominal radial resolution as fine as 10-20 m (Esposito et al. 2004; Colwell et al. 2010; Jerousek et al. 2016).

In addition to the above purpose-built instruments, both of Cassini's infrared instruments, the Visual and Infrared Mapping Spectrometer (VIMS) and the Composite Infrared Spectrometer (CIRS), were used to carry out stellar occultation experiments using bright, late-type stars. It is the purpose of this paper to describe how the VIMS instrument was used to obtain occultation data, and to document the photometric and geometric calibrations necessary to derive radial profiles of ring optical depth in the near-infrared. Approximately 180 such profiles were obtained over the 13-year span of Cassini's orbital tour, ranging in resolution from $\sim 150 \mathrm{~m}$ to $\sim 1 \mathrm{~km}$. For most of these occultations standardized optical depth profiles have been delivered to the Planetary Data System's Ring Moon Systems Node, and an additional purpose of this paper is to provide suitable documentation for these data.

Previous papers have presented specific scientific investigations of Saturn's rings based entirely or primarily on the VIMS stellar occultation data, including studies of self-gravity wakes in the A and B rings (Hedman et al. 2007; Nicholson and Hedman 2010), the structure of the Cassini Division (Hedman et al. 2010), viscous overstability in the inner A ring (Hedman et al. 2014), waves in the $\mathrm{C}$ ring driven by saturnian internal oscillations (Hedman and Nicholson 2013, 2014), the bending wave driven at the Titan -1:0 resonance (Nicholson and Hedman 2016) and the surface mass density of the B ring (Hedman and Nicholson 2016). In addition, a series of four papers has documented the shapes of noncircular features 
throughout Saturn's rings using data from the RSS, UVIS and VIMS occultations (Nicholson et al. 2014a,b; French et al. 2016a, 2017). All of these papers depend on the data described herein, and on its geometric and photometric calibration.

Although the present paper is largely directed towards ring stellar occultations, we include in Section 8 a discussion of 30 solar occultations by the rings observed by the VIMS instrument. Relatively few papers have been published based on these data, but it is hoped that this situation will change if the existence of these data sets is more widely known. The Cassini-VIMS instrument was also used to observe both stellar and solar occultations by the atmospheres of Saturn and Titan. These data will form the subject of a future publication, as they involve very different scientific goals and distinct observational protocols and calibration techniques.

\section{Observations}

\subsection{Standard imaging operations}

In order to understand how VIMS observed occultations, it is useful first to review how the instrument operates in its more normal spectral imaging mode. ${ }^{1}$ The design, principal characteristics and operational modes of the Cassini VIMS instrument are described in some detail by Brown et al. (2004), along with the procedures used for pre-launch calibrations. We will simply summarize the salient points here, and direct the reader to this work for more extensive information, instrument schematics, etc.

VIMS is an imaging spectrometer, designed primarily to produce spectrally-resolved images of targets in the Saturn system over the wavelength range 0.35 to $5.1 \mu \mathrm{m}$. This is achieved with two co-aligned optical systems: a visual (VIS) channel equipped with a diffraction grating and a $288 \times 576$ pixel CCD detector that generates $2 \mathrm{D}$ spatial-spectral images with 64 spatial pixels at $0.35-1.1 \mu \mathrm{m}$, and a near-infrared (IR) channel with a diffraction grating and a 256 element linear InSb detector that generates single-pixel spectra from 0.88 to $5.11 \mu \mathrm{m}$. In normal operations, a 1D scanning mirror in the VIS channel and a coordinated 2D scanning mirror in the IR channel are used to synthesize 3D hyper-spectral 'cubes' of the target scene, with $64 \times 64$ spatial pixels and 352 spectral channels. Each standard pixel (or IFOV) is $0.5 \times 0.5 \mathrm{mrad}$ in dimension and the full spectrum is divided between 96 VIS channels and 256 IR channels, with a small overlap around $1 \mu \mathrm{m}$. Internal timing signals are used to ensure that the IR channel obtains a single line of 64 pixels in the same time that the VIS channel acquires a single 2D CCD exposure.

The instrument's fast-scan direction is towards $+\mathrm{X}$ (as defined by the standard Cassini body-fixed coordinate system), while the slow-scan direction is towards $+Z$. The instrument's nominal boresight points in the $-\mathrm{Y}$ direction, and is approximately aligned with that of the other three Cassini optical remote sensing instruments. In order to facilitate

\footnotetext{
${ }^{1}$ VIMS, of course, no longer exists following the deliberate de-orbiting of Cassini on 15 September 2017.
} 
accurate flux measurements, at the end of each IR line (or each VIS exposure), a shutter is closed in each channel and a series of background measurements (1, 2 or 4 integrations) is made and recorded. These serve to monitor the instrumental dark current, the thermal background in the IR, and any electronic offsets in the data processing chain. This average background spectrum is then subtracted from each line of data before it is compressed and sent to the spacecraft's central processing unit for eventual transmission to the Earth. However, the background spectrum is also transmitted, so that if necessary the entire process can be 'undone' on the ground. This latter precaution turns out to be quite useful for occultation data, as described in Section 3 below.

The VIMS instrument is quite flexible, with both the width $(\mathrm{X})$ and height $(\mathrm{Z})$ of the recorded cubes being adjustable, up to a maximum of 64 pixels, as are the independentlycommanded VIS and IR integration times. For smaller cubes, the location of the recorded image within the full $64 \times 64$ pixel field of view may be specified arbitrarily. ${ }^{2}$ The number of spectral channels returned can also be selectively reduced, subject to the constraint that there are a multiple of 32 VIS channels and a multiple of 32 IR channels. If it is necessary to reduce data volume even further, the raw spectra can be co-added in groups of 8 adjacent channels. Finally, both VIS and IR channels also have 'high spatial resolution' modes, which may be invoked as needed. For the VIS channel the pixel size is reduced by a factor of $\frac{1}{3}$ in both dimensions, while the IR pixel size is reduced to $0.25 \mathrm{mrad}$ in $\mathrm{X}$ but remains unchanged in $\mathrm{Z}^{3}$ The maximum image size remains $64 \times 64$ pixels.

\subsection{Occultation mode}

When used in stellar occultation mode, a specific instrument configuration has been used throughout the mission that involves a standard setup command and only a minimal number of adjustable parameters. Since the goal here is to measure the brightness of a point source (the occulted star) as frequently as is feasible, while maximizing the signal-to-noise ratio of the data, occultation mode disables the spatial-imaging capability of the instrument by holding the $2 \mathrm{D}$ scanning mirror in the IR channel fixed at a predetermined location (see below). This is feasible because the core of the point spread function of the IR channel, as calculated from the instrument's optical design and also measured in-flight with stellar observations, is significantly smaller than 1 pixel. In order to minimize any background signal (e.g., from the rings or scattered light from Saturn), the instrument is operated in high-resolution mode, with its native IR pixel size of $0.25 \times 0.5 \mathrm{mrad}$. The VIS channel, which is less sensitive in point mode than is the IR channel, and operates more slowly, is turned off. For ring stellar occultations, where the transmission of the rings is expected to be gray, or only slowly-varying in wavelength, the data are almost always spectrally-summed,

\footnotetext{
${ }^{2}$ Subject to the constraint that only even offsets in $\mathrm{Z}$ are permitted.

${ }^{3}$ In normal, or 'low-res' mode, the standard $0.5 \times 0.5 \mathrm{mrad}$ IR IFOV is actually synthesized by combining two adjacent hi-res measurements. The $0.25 \mathrm{mrad}$ width of the hi-res pixel is set by the width of the spectrometer's entrance slit, while its $0.50 \mathrm{mrad}$ height is set by the physical dimension of the IR pixels.
} 
thus reducing the compressed data volume by a factor of $\sim 5$. IR integration times, which are adjusted according to the brightness of the target star and its projected radial velocity across the rings, range from $20 \mathrm{~ms}$ to $100 \mathrm{~ms}$.

A critical element in obtaining useful occultation data for either rings or atmospheres is to have accurate absolute timing knowledge for each sample measurement, something which unfortunately was not achieved for the Voyager stellar occultation experiments (Nicholson et al. 1990). To this end, a timing signal derived from the instrument's own internal clock is added to each recorded spectrum, taking the place of the final 8 spectral channels. This internal timer runs at approximately $11 \mathrm{kHz}$, providing relative timing precision of $<1 \mathrm{~ms}$, and once per second it is synchronized with the spacecraft's master clock, whose time system is known as SCLOCK. By regular comparisons between the spacecraft and Deep Space Network station clocks (atomic clocks which are linked to Ephemeris Time, or ET), a file of corrections from SCLOCK to both ET and UTC, as measured on Cassini, is maintained and disseminated by the Cassini project.

Every 64 samples, the continuous recording of occultation data is interrupted by up to 4 integration periods to obtain a background measurement. As in normal operations, the average background spectrum is then subtracted from the data before they are compressed and subsequently transmitted to Earth. To improve operational efficiency, occultation data are packaged on board into $64 \times 64$ pixel cubes, though in fact the scanning mirror remains fixed throughout the observation. Raw VIMS stellar occultation data are always stored in $64 \times 64$ cube format, with the corresponding background data stored in the sideplane of the cube, with one average background measurement for each wavelength and each line of the cube. Ground software extracts the timing data from the last 8 spectral channels and places the start time of each cube into the header, in SCLOCK, SCET and ET formats, but the original SCLOCK time remains embedded in the data for use by the data analyst.

\subsection{Stellar acquisition}

Were Cassini able to point its instruments at a specified celestial position (i.e., a star) with an accuracy significantly less than 1 VIMS pixel, then occultation observations would be straightforward. In reality, however, the spacecraft's a priori control pointing error was specified at $2.0 \mathrm{mrad}(99 \%)$, or 4 standard VIMS pixels, and it was necessary to devise a method for on-board acquisition of stellar targets. In practice, the measured 3- $\sigma$ targeting accuracy, or 'control error', of Cassini is much better than the specifications, and was found to be $\sim 0.4 \mathrm{mrad}$ about the $\mathrm{X}$ axis and $\sim 0.6 \mathrm{mrad}$ about $\mathrm{Z}$ (Pilinsky and Lee 2009). This is achieved using a combination of Cassini's three reaction wheels (RWAs) and its two CCDbased Stellar Reference Units (SRUs), or star-trackers, and the combined or radial error of 0.6 mrad corresponds to $\sim 100$ pixels in the Narrow Angle Camera (NAC) or a little over 
one standard pixel for VIMS. ${ }^{4}$ While it is thus impossible to predict in which VIMS pixel the stellar image will fall, the $3-\sigma$ uncertainty is at most 2 high-resolution pixels in X and 1 pixel in Z. Furthermore, the spacecraft pointing is extremely stable once a new target is acquired by the star trackers. On-orbit tests show that the RMS pointing variations are $4-5 \mu \mathrm{rad}$ over periods of up to $20 \mathrm{~min}$ (Pilinsky and Lee 2009), or $\ll 1$ VIMS pixel. In practice, VIMS has observed occultations with durations exceeding $12 \mathrm{hr}$, without any evidence for a degradation in the pointing stability.

To solve the problem of initial targeting on board (the two-way light travel time to Saturn is $\sim 150 \mathrm{~min}$, which precludes human intervention), the VIMS instrument was programmed to first obtain a small image of the star shortly before the predicted start of the occultation. Hardware constraints required this image to contain exactly 64 pixels. The instrument's internal data compression software then identifies the brightest pixel in the scene (assumed to be the star of interest), and uses this to calculate the 2D mirror offset necessary to place the star in the single pixel to be observed for the remainder of the occultation period. Although the dimensions of this so-called star-finding cube were set in software, rather than being hard-wired into the VIMS signal processing electronics, in practice they were fixed at $16 \times 4$ pixels early in the mission and have never been changed. ${ }^{5}$ Operationally, the stellar image only rarely falls more than 1 or 2 pixels off-center in the $\mathrm{X}$ direction in the star-finding cube, and never more than 1 pixel away in $\mathrm{Z}$. But with a Z-dimension of only 4 pixels, the star not infrequently falls in the top or bottom row of the cube and very occasionally may fall beyond this. In cases where the stellar image falls outside the star-finding cube, the scanning mirror will be set to the wrong pixel and the stellar signal is either greatly reduced or lost entirely. (The latter happened only a few times in the entire mission.)

Fig. 1a illustrates a typical star-finding cube obtained during an occultation of $\gamma$ Crucis, while Fig. 1b indicates where the default field of view of the star-finding cube falls within the central portion of the VIMS FOV. (In operations, this default pointing was tweaked slightly to match the predicted spacecraft pointing profile for each observation sequence.) About $85 \%$ of the time, the star was found to fall in hi-res pixel [62,31], indicated by the asterisk in the figure, which we therefore take to be the actual location of the VIMS boresight vector as defined in the spacecraft's pointing kernel. (Hi-res pixels are counted from $0-127$ in $\mathrm{X}$ and $0-63$ in $\mathrm{Z}$, with the instrument's nominal (design) boresight being hi-res pixel $[64,32]$.$) The star-finding cube is also returned to the ground, should it be$ necessary to examine this later, as are the $2 \mathrm{D}$ scanning mirror coordinates selected by the on-board star-finding software.

For the reader interested in re-analyzing the VIMS occultation data, we note that

\footnotetext{
${ }^{4}$ Comparisons of predicted and actual images of stars and small satellites suggest that the actual error achieved was closer to $20 \mathrm{NAC}$ pixels or $\sim 0.12 \mathrm{mrad}$ (M. Evans, private communication).

${ }^{5}$ The longer dimension was chosen to be in the $\mathrm{X}$ direction, both because the hi-res pixels are smaller in this direction and because it was initially believed that the pointing accuracy of the SRUs would be lower in $\mathrm{X}$ than in Z. In reality this is not true.
} 
the targeting of smaller lo-res VIMS cubes is achieved simply by specifying the $\mathrm{X}$ and $\mathrm{Z}$ coordinates of the upper-left corner of the desired field, denoted by the parameters $\mathrm{X}_{\text {off }}$ and $\mathrm{Z}_{\text {off }}$. For hi-res cubes, on the other hand, the initial scan mirror position in $\mathrm{X}$ is given by the expression $\mathrm{X}_{\text {hires }}=2 \times \operatorname{INT}\left[\mathrm{X}_{\text {off }}+N_{x} / 4\right]$, where $N_{x}$ is the $\mathrm{X}$-dimension of the cube in hi-res pixels and the function INT denotes 'the integer part of'. This ensures that the hi-res cube is centered at the same position as the corresponding lo-res cube with the same offsets.

For future reference we note here that the boresight of the VIMS solar port - used for the solar occultations discussed in Section 8 below — is located at lo-res pixel $[29,30]$.

Although this simple procedure has its limitations, chiefly that it does not cope well with situations where the star falls more or less midway between two pixels, in practice it has worked well in $\sim 90 \%$ of the occultations we have attempted. In only 4 cases out of 190 ring occultations did VIMS fail to acquire the star, or lose it after the initial acquisition. In a further 11 cases - or $\sim 6 \%$ of the time - the occultation was recorded but the stellar signal was observed to be less than one-third of the predicted level, suggesting that the stellar image was not in fact centered within the pixel selected by the onboard algorithm. (See Fig. 12 below and the associated discussion in Section 6 for further details.) In most of these cases, the measured stellar signal level outside the occultation period is quite variable, consistent with the hypothesis that the mirror was set on a pixel adjacent to the star, or that the star's flux was divided more-or-less equally between two or more adjacent pixels. In the latter situation clearly no single choice of pixel would have worked well.

One might ask, given the a priori pointing uncertainties of $\sim 0.5 \mathrm{mrad}$ and our inability to make sub-pixel pointing adjustments on-board, why the vast majority of VIMS stellar occultations returned data of good quality. The answer appears to lie in the relatively uniform spatial response across each of the VIMS IR detectors, and the small size of the gaps between pixels. In order to measure the spatial response of the IR detectors, several in-flight calibrations were carried out in which a bright star was moved in a slow raster-scan pattern across the VIMS boresight, while taking data continuously in occultation mode. An example of such an observation is shown in Fig. 2, for wavelengths between 1.3 and $4.0 \mu \mathrm{m}$. We see that the typical detector response function is quite flat-topped, especially at shorter wavelengths and in the X-direction, with relatively sharp edges. Only for scans which barely clipped one end of the pixel (indicated by lower peak signal levels) do we see a significantly rounded profile. Furthermore, measurements of the FWHM of many such scans show that the effective dimensions of the hi-res pixel are $0.23 \mathrm{mrad}$ (in $\mathrm{X}$ ) by $0.49 \mathrm{mrad}$ (in $\mathrm{Z}$ ), in good agreement with the instrument's optical design specifications and very close to the interpixel spacing of 0.25 by $0.50 \mathrm{mrad}$ measured in ground calibrations (Brown et al. 2004). We conclude that the inter-pixel gaps are no larger than $10 \%$ in $\mathrm{X}$ and even smaller in Z.

From these observations, we conclude (a) that the chance of the stellar image falling in the gap between 2 pixels is $\sim 10 \%$, on average, and (b) that the probability that the measured flux will be at least one-half of the maximum value is close to $90 \%$. This is, in 

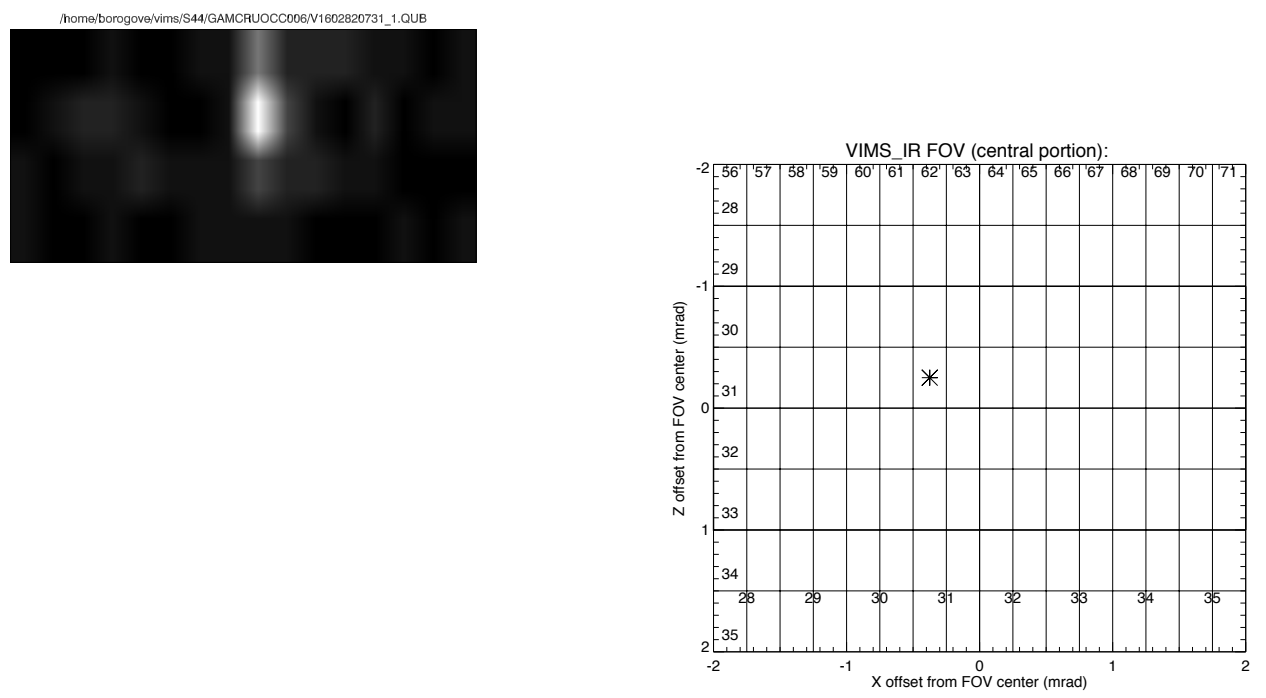

$X($ hires $)=2$ *fix $[X o f f+N \times / 4] \quad$ starcal_occ_fov8.pro: Mon Aug 14 00:43:29 2017

Fig. 1. - To the left is an example of a typical $16 \times 4$-pixel star-finding cube recorded prior to the occultation of $\gamma$ Crucis on rev 89. Note that in high-resolution mode, VIMS pixels are rectangular with dimensions of $0.25 \mathrm{mrad}$ (in X) by $0.50 \mathrm{mrad}$ (in Z). The star here is in pixel $[8,2]$, using the standard VIMS 0-based counting scheme with $\mathrm{X}$ increasing to the right and $\mathrm{Z}$ increasing upwards. To the right is a diagram of the central portion of the full VIMS high-resolution field of view, as projected on the sky. The geometric center of the VIMS FOV is at the origin. Labels on the left side denote pixel coordinates in Z (numbered 0-63, increasing downwards). Labels along the top denote hi-res pixel coordinates in X (numbered 0-127, increasing to the right), while those along the bottom indicate the corresponding lo-res pixel coordinates (0-63). Each lo-res pixel represents the sum of two adjacent hi-res pixels. The nominal VIMS-IR boresight, as derived from many occultation observations, corresponds to hi-res pixel $[62,31]$, denoted by the asterisk. Heavier lines show the default targeting of the $16 \times 4$-pixel star-finding cube, with $\mathrm{X}_{\text {off }}=24$ and $\mathrm{Z}_{\text {off }}=30$ (see text). 
fact, in reasonable agreement with what we see in Fig. 12 below.

\section{Occultation geometry}

\subsection{Spatial resolution}

As is the case for ground-based stellar occultations, occultations observed by spacecraft are limited in their spatial resolution not by the angular resolution of the instrument but by some combination of its temporal sampling rate, Fresnel diffraction and the angular diameter of the occulted star (Elliot 1979; Nicholson et al. 1982). Typical radial velocities for Cassini stellar occultations are $5-10 \mathrm{~km} \mathrm{~s}^{-1}$, with significantly lower or higher values occurring for some very distant or unusual geometries. At a $40 \mathrm{~ms}$ integration time, this translates into a radial sampling interval of 200-400 m. For the brightest stars, observed at $20 \mathrm{~ms}$ integration time, the VIMS radial sampling interval can be as small as $150 \mathrm{~m}$.

A shorter integration time, however, would not necessarily improve the spatial resolution. Fundamentally this is set by Fresnel diffraction (see, e.g., Nicholson et al. (1982) for models) and/or the projected linear diameter of the occulted star. At a range $D$ from the spacecraft to the rings, and at an observing wavelength $\lambda$, the apparent width of an occultation profile for an infinitely-sharp edge is limited by diffraction to about twice the Fresnel zone, or $\sqrt{2 \lambda D}$. For VIMS ring occultations at $2.92 \mu \mathrm{m}$, this is $45 \mathrm{~m}$ at $D \simeq 6 R_{S}$, increasing to $105 \mathrm{~m}$ at $D \simeq 30 R_{S}$. A ringlet or a gap narrower than this may be detected but will not be properly resolved. Finally there is an additional 'smoothing' of the light curves due to the finite angular diameter of the occulted star. Many bright stars in the near-infrared are late-type giants or supergiants, with substantial sizes. Typical angular diameters are in the range 5-50 mas, as measured by interferometric techniques, which correspond to projected linear diameters at the rings of 10-100 $\mathrm{m}$ at $D \simeq 6 R_{S}$, or up to $350 \mathrm{~m}$ at $D \simeq 30 R_{S}$ (see Table 10 in the Appendix.)

For most VIMS occultations, the radial resolution is limited by the integration time, but in a significant minority of cases the stellar diameter becomes the limiting factor. In a few cases with favorable geometry, this has even permitted the stellar diameter to be probed as a function of wavelength by analyzing the sharpness of occultation profiles (Stewart et al. 2016a,b).

\subsection{Predictions}

Predictions for ring occultations observable by Cassini were generated by B. Wallis at the Jet Propulsion Laboratory, using the predicted spacecraft trajectory and a catalog of $\sim 150$ bright stars in the near-infrared. The latter was derived from the standard IR star catalog used for calibrations at Palomar Observatory, as maintained by K. Matthews, with a cutoff at a magnitude limit of $K=0$. This list was augmented by a few dozen bright southern calibrator IR stars drawn from the literature, plus several bright point sources 

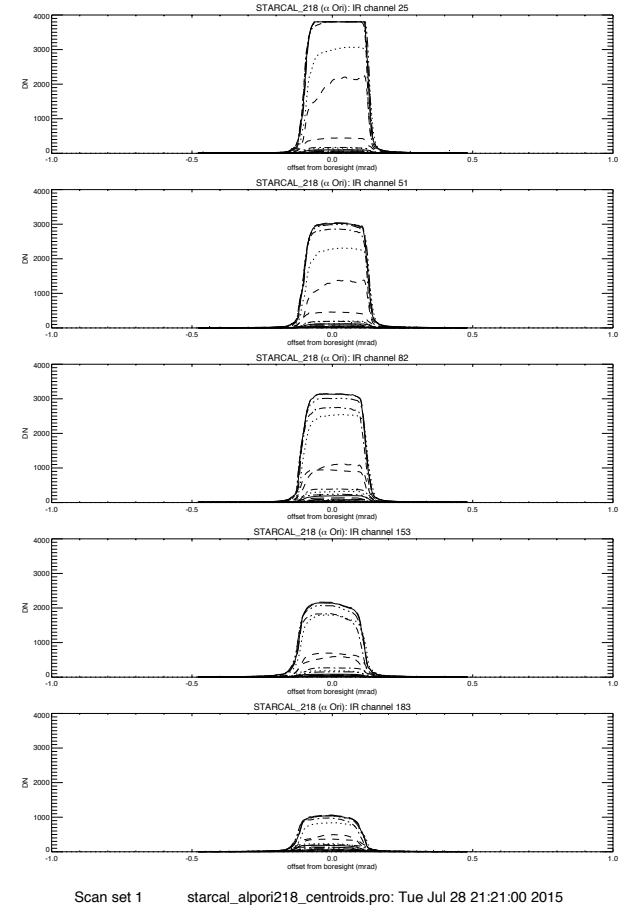
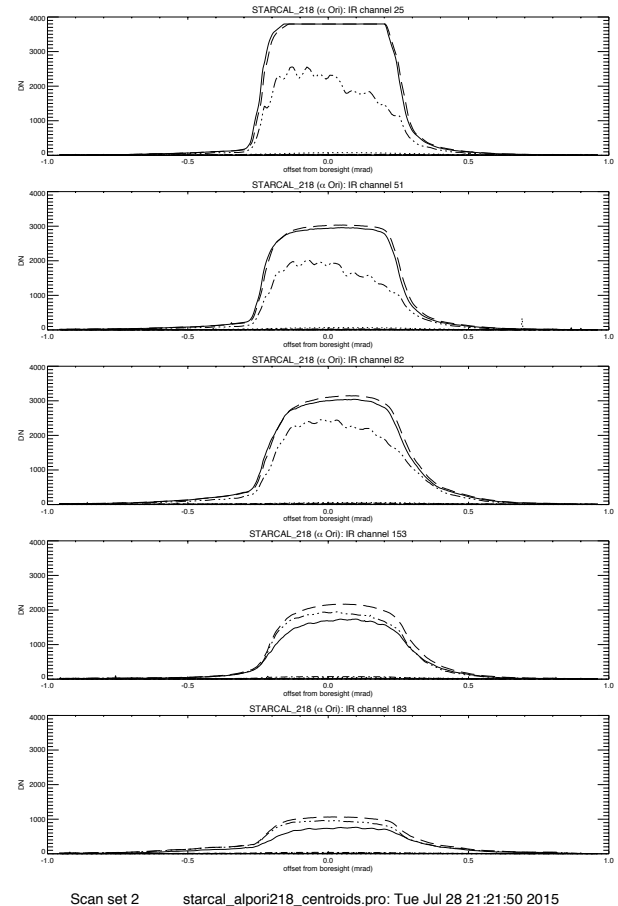

Fig. 2.- A sequence of drift scans across the star $\alpha$ Orionis obtained during a stellar calibration observation on rev 218. VIMS was operated in occultation (i.e., single-pixel, high-resolution) mode, while Cassini was commanded to carry out two series of slow scans of the star across the VIMS boresight, each in the form of a raster scan pattern covering a region $0.5 \times 1.0 \mathrm{mrad}$ in size on the sky. The left column shows a sequence of 21 scans in the $\mathrm{X}$ direction, with $\mathrm{Z}$ offsets varying from -1.0 to $+1.0 \mathrm{mrad}$, while the right column shows a similar sequence of 11 scans in the $\mathrm{Z}$ direction, with $\mathrm{X}$ offsets varying from -0.5 to $+0.5 \mathrm{mrad}$. In each case, the spacing between scans was $0.1 \mathrm{mrad}$, which is the smallest pointing offset commandable for Cassini. The spectrum of the star was measured every $320 \mathrm{~ms}$, and data are shown here at wavelengths of $1.30,1.72,2.23,3.42$ and $3.92 \mu \mathrm{m}$, from top to bottom. The sharper edges of the pixel in the X-direction are defined by the edges of the spectrometer slit in the VIMS telescope's focal plane (Brown et al. 2004), while the 'fuzzier' edges in the Z-direction are set by the physical dimensions of the detector and the spectrometer's point spread function. Note that the stellar signal saturates at $3800 \mathrm{DN}$ at wavelengths shortward of $\sim 1.4 \mu \mathrm{m}$. 
from the 2 Micron Sky Survey and IRAS catalogs. In practice, a subset of $\sim 40$ stars was used for all the ring occultations actually observed, due to the repetitive nature of the Cassini trajectory and the clustering of bright stars on the sky.

A complete list of the 47 stars used for all VIMS occultations (including those by Saturn and Titan) is provided in Table 1, along with their magnitudes, spectral types, saturnicentric latitudes $B_{*}$ and longitudes $\lambda_{*}$ and estimated angular diameters $\theta_{*}$ (see discussion of occultation geometry in Section 5). The distribution of these stars on the saturnian sky is shown in Fig. 3. Note that $B_{*}$ is the inclination of the stellar line-of-sight to Saturn's ring plane, so that we will also refer to $\left|B_{*}\right|$ as the ring opening angle for an occultation observation.

For each potential event, the start and end times were calculated, using as boundaries the $\mathrm{F}$ ring at a radius from Saturn of $140,200 \mathrm{~km}$ and the innermost feature in the D ring at $68,000 \mathrm{~km}$. Ingress and egress occultations were treated as separate events, as it was only rarely possible to observe both given other spacecraft scheduling constraints. Because of the necessity to obtain a star-finding cube before each occultation, VIMS cannot observe occultations which start with the star emerging from behind the planet, unless there is a sufficient gap between Saturn egress and entry into the C ring. For this reason, observations of egress occultations are quite rare.

A substantial fraction of the ring occultations actually observed fall in neither the ingress or egress category. Instead, the star followed a diagonal path across one ring ansa, thus providing two cuts across each ring radius probed, down to some minimum value, known as the 'turn-around' radius. Such events we refer to as chord occultations; they are particularly common when Cassini is on high-inclination orbits but occur only rarely in earth-based occultations. Chord occultations are associated with variable but often quite low radial velocities, as projected into the ring plane, and tend to have the highest radial resolution of our observations, especially near the turn-around radius. Although no serious attempt was made to choose VIMS occultations with turn-around radii in specific locations in the rings, we do take note of these radii in the tables of data presented below.

In several cases an occultation ended prematurely when the star was occulted by Saturn before it reached the $\mathrm{D}$ ring, with the radial profile ending in the $\mathrm{C}$ - or even $\mathrm{B}$ - ring. In two cases a deep chord occultation was interrupted briefly by a grazing Saturn occultation, resulting in some loss of data in the $\mathrm{C}$ and $\mathrm{D}$ rings.

A typical VIMS radial stellar occultation has a duration of $3 \mathrm{hrs}$, corresponding to $\sim 250,000$ measurements of the stellar spectrum at an integration time of $40 \mathrm{~ms}$. Each (summed) IR spectrum consists of 256/8 = 32 spectral samples, recorded at 12 bits/sample, for a total volume of $\sim 96$ Mbits, uncompressed, or $\sim 40$ Mbits with the standard lossless compression algorithm employed by VIMS. But several slower events were also observed, with durations of 5-10 hrs. The longest VIMS ring occultations observed are distant chord events, monitored when the spacecraft velocity was unusually slow, which have durations ranging from 16 to $24 \mathrm{hrs.}$. 
Table 1: Catalog of stars used in VIMS occultations.

\begin{tabular}{|c|c|c|c|c|c|c|c|c|}
\hline $\mathrm{BS}^{a}$ & Name & Sp. type & $K$ mag & $\begin{array}{l}\text { R.A. }{ }^{b} \\
(\mathrm{deg})\end{array}$ & $\begin{array}{l}\text { Dec. }{ }^{b} \\
\text { (deg) }\end{array}$ & $\begin{array}{r}B_{*}^{c} \\
(\operatorname{deg})\end{array}$ & $\begin{array}{r}\lambda_{*}^{c} \\
(\operatorname{deg})\end{array}$ & $\begin{array}{r}\theta_{*}^{d} \\
(\mathrm{mas})\end{array}$ \\
\hline 337 & betAnd & M0III & -1.87 & 17.4329 & 35.6206 & 41.52 & 244.74 & 12.2 \\
\hline 681 & omiCet & M7e & -2.60 & 34.8363 & -2.9775 & 3.45 & 264.25 & 29: \\
\hline 911 & alpCet & M2III & -1.68 & 45.5696 & 4.0897 & 10.53 & 275.06 & 11.6 \\
\hline 921 & rhoPer & M4II & -1.93 & 46.2938 & 38.8403 & 45.27 & 276.33 & 15.0 \\
\hline 1231 & gamEri & M0III & -0.93 & 59.5071 & -13.5086 & -7.39 & 288.54 & 8.5 \\
\hline 1457 & alpTau & K5III & -2.80 & 68.9800 & 16.5092 & 22.17 & 299.50 & 21.3 \\
\hline \multirow[t]{2}{*}{1492} & R Dor & M8III & -3.41 & 69.1900 & -62.0775 & -56.27 & 293.82 & $(57)$ \\
\hline & TX Cam & M9III & -0.01 & 75.2099 & 56.1813 & 61.29 & 311.18 & $5.5^{e}$ \\
\hline 1693 & RX Lep & M6III & -1.26 & 77.8450 & -11.8492 & -6.68 & 306.63 & \\
\hline 1708 & alpAur & $\mathrm{G} 8 ?+\mathrm{F}$ & -1.81 & 79.1721 & 45.9981 & 50.88 & 313.38 & (5.2) \\
\hline 2061 & alpOri & M1Ia & -4.00 & 88.7929 & 7.4069 & 11.68 & 319.03 & 37.5: \\
\hline 2491 & alpCMa & $\mathrm{A} 1 \mathrm{~V}$ & -1.36 & 101.2871 & -16.7161 & -13.48 & 329.20 & (5.6) \\
\hline \multirow[t]{2}{*}{2748} & L2 Pup & M5III & -1.78 & 108.3846 & -44.6397 & -41.91 & 332.28 & \\
\hline & VY CMa & M5eIb & -0.69 & 110.7466 & -25.7688 & -23.43 & 337.41 & 18.7 \\
\hline 2943 & alpCMi & F5IV & -0.65 & 114.8254 & 5.2250 & 6.95 & 344.91 & 5.4 \\
\hline 3634 & lamVel & K4Ib & -1.53 & 136.9988 & -43.4325 & -43.81 & 0.26 & $(12.5)$ \\
\hline 3639 & RS Cnc & M6III & -1.64 & 137.6608 & 30.9631 & 29.95 & 10.84 & 15 \\
\hline 3748 & alpHya & K4III & -1.19 & 141.8967 & -8.6586 & -9.87 & 10.28 & (9.1) \\
\hline 3816 & R Car & M6III & -2.05 & 143.0612 & -62.7886 & -63.48 & 359.76 & (20) \\
\hline \multirow[t]{3}{*}{3882} & R Leo & M8III & -3.21 & 146.8892 & 11.4286 & 9.55 & 17.46 & $28:$ \\
\hline & CW Leo & C6 & 1.31 & 146.9862 & 13.2786 & 11.38 & 17.76 & 52 \\
\hline & etaCar & pec & 1.14 & 161.2619 & -59.6858 & -62.47 & 20.09 & var. \\
\hline 4267 & $56 \mathrm{Leo}$ & M5III & -0.80 & 164.0058 & 6.1853 & 2.60 & 33.84 & $4^{e}$ \\
\hline 4671 & epsMus & M5III & -1.42 & 184.3925 & -67.9606 & -72.77 & 41.59 & $13^{e}$ \\
\hline 4763 & gamCru & M3III & -3.04 & 187.7912 & -57.1131 & -62.35 & 50.68 & 24.4 \\
\hline
\end{tabular}

${ }^{a}$ Number in Yale Bright Star Catalog; unnumbered stars denote sources from the $2 \mu \mathrm{m}$ Infrared Catalog or the IRAS Point Source Catalog.

${ }^{b}$ Heliocentric coordinates, J2000 (BSC).

${ }^{c}$ Saturnicentric latitude \& longitude.

${ }^{d}$ Angular diameter at $K$-band; values in () were measured at other wavelengths. A : indicates an uncertain value.

${ }^{e}$ Estimated value based on magnitude and spectral type. 


\begin{tabular}{|c|r|c|r|r|r||r|r|r|}
\hline BS $^{a}$ & Name & Sp. type & $K$ mag & $\begin{array}{r}\text { R.A. }^{b} \\
(\mathrm{deg})\end{array}$ & $\begin{array}{r}\text { Dec. } \\
(\mathrm{deg})\end{array}$ & $\begin{array}{r}B_{*}^{c} \\
(\mathrm{deg})\end{array}$ & $\begin{array}{r}\lambda_{*}^{c} \\
(\mathrm{deg})\end{array}$ & $\begin{array}{r}\theta_{*}^{d} \\
(\mathrm{mas})\end{array}$ \\
\hline 4910 & delVir & M3III & -1.25 & 193.9004 & 3.3975 & -2.38 & 63.35 & 9.8 \\
5080 & R Hya & M7III & -2.66 & 202.4279 & -23.2811 & -29.41 & 70.82 & 25 \\
& W Hya & M8e & -3.10 & 207.2605 & -28.3680 & -34.65 & 75.74 & 40 \\
5192 & 2 Cen & M5III & -1.68 & 207.3608 & -34.4506 & -40.73 & 75.59 & 14.7 \\
5340 & alpBoo & K2III & -2.99 & 213.9150 & 19.1825 & 12.76 & 83.55 & 20.2 \\
& & & & & & & & \\
5459 & alpCen & G2V & -1.48 & 219.9008 & -60.8353 & -67.30 & 89.14 & 8.3 \\
6056 & delOph & M1III & -1.22 & 243.5858 & -3.6944 & -9.64 & 113.30 & 9.3 \\
6134 & alpSco & M1Iab & -3.78 & 247.3517 & -26.4319 & -32.16 & 118.46 & $40:$ \\
6146 & 30 Her & M6III & -2.01 & 247.1600 & 41.8817 & 36.04 & 114.33 & 14.8 \\
6217 & alpTrA & K2II & -1.20 & 252.1663 & -69.0278 & -74.18 & 133.46 & $12^{e}$ \\
& & & & & & & & \\
6406 & alpHer & M5II & -3.37 & 258.6617 & 14.3903 & 9.27 & 127.25 & 34 \\
7001 & alpLyr & A0V & 0.02 & 279.2342 & 38.7836 & 35.22 & 144.58 & 3.3 \\
7002 & X Oph & K1III & -0.90 & 279.5871 & 8.8339 & 5.47 & 148.31 & 13.2 \\
7157 & R Lyr & M5III & -2.09 & 283.8333 & 43.9461 & 40.78 & 148.11 & 14.3 \\
7243 & R Aql & M7III & -0.57 & 286.5921 & 8.2300 & 5.56 & 155.30 & 10.6 \\
& & & & & & & & \\
8308 & epsPeg & K2I & -0.81 & 326.0463 & 9.8750 & 11.54 & 194.28 & 7.5 \\
8316 & mu Cep & M2Ia & -1.65 & 325.8762 & 58.7800 & 59.90 & 184.53 & 14.1 \\
8636 & betGru & M5III & -3.22 & 340.6667 & -46.8847 & -43.38 & 215.55 & $(27)$ \\
8775 & betPeg & M2II & -2.22 & 345.9433 & 28.0828 & 31.68 & 212.27 & 15 \\
8850 & chiAqr & M3III & -0.21 & 349.2117 & -7.7267 & -3.67 & 219.13 & $(6.7)$ \\
& & & & & & & & \\
9066 & R Cas & M7III & -1.84 & 359.6029 & 51.3886 & 56.04 & 222.89 & 25 \\
9089 & 30 Psc & M3IV & -0.47 & 0.4896 & -6.0142 & -1.06 & 230.17 & 7.2 \\
\hline
\end{tabular}




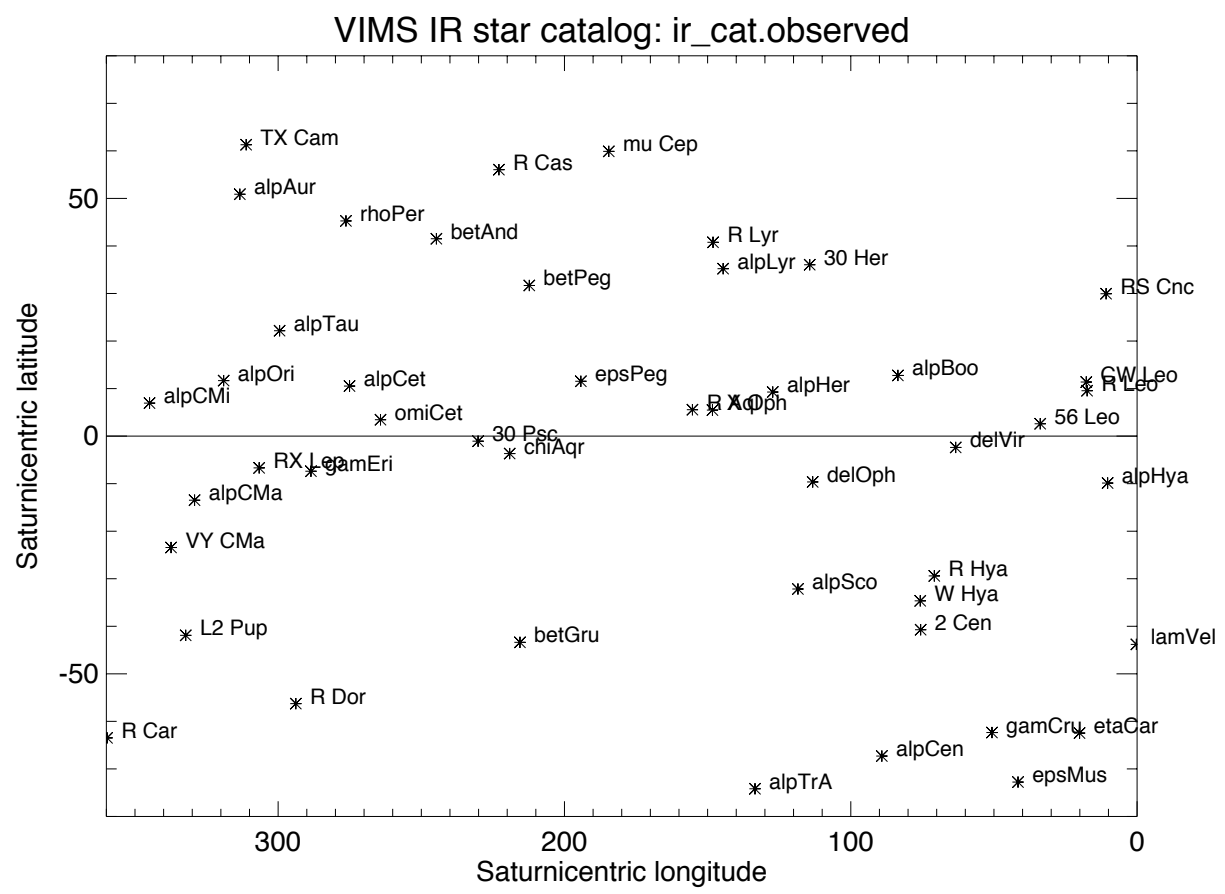

Fig. 3.- Distribution on the sky of the stars used for occultation observations by CassiniVIMS during the course of its 13-year orbital tour. The plotted positions are the saturnicentric latitudes, $B_{*}$, measured from Saturn's equator, and saturnicentric longitudes, $\lambda_{*}$, measured from the ascending node of Saturn's equator on the Earth's equator of J2000, as given in Table 1. 


\section{Overview of VIMS ring occultations}

A complete list of all 190 ring stellar occultations observed (or attempted) with Cassini VIMS is provided in the Appendix as Table 8, including the star name and Cassini orbit number (or 'rev'), the start time and duration of each observation, the integration time used, whether or not the data were spectrally-summed or edited and the measured signal level for the unocculted star. Additional notes provide a shorthand description of the radial coverage of the occultation. A more detailed discussion of the entries in this table may be found in Section 7 below.

An example of a high-quality, complete radial occultation of the bright star $\gamma$ Crucis is shown in Fig. 4, both in raw form and converted to optical depth. The data are plotted as a function of radius in Saturn's equatorial plane (see Section 5). Note that the stellar flux has not been normalized, in order to show the actual recorded signal level. In this case, the unocculted stellar count rate was $720 \mathrm{DN}$ per $40 \mathrm{~ms}$ sample, or 18,000 DN per second, co-added over 8 spectral channels. An example of a high-quality chord occultation is shown in Fig. 5, in this case of our brightest star $\alpha$ Orionis (Betelgeuse). Here the unocculted stellar count rate was $950 \mathrm{DN}$ per $20 \mathrm{~ms}$ sample, or 47,500 DN per second.

Saturn's rings are conventionally divided into three main sub-regions: the A ring, between radii of $136,700 \mathrm{~km}$ and $122,000 \mathrm{~km}$, the $\mathrm{B}$ ring between $117,500 \mathrm{~km}$ and $92,000 \mathrm{~km}$, and the $\mathrm{C}$ ring between $92,000 \mathrm{~km}$ and $74,500 \mathrm{~km}$. In both Figs. 4 and 5 - even at their compressed scale - we can see the sharp inner and outer edges of the A and B rings, as well as identify the narrow Encke and Keeler gaps in the outer A ring, at radii of 133,500 and 136,000 km, respectively. The central B ring, between radii of 104,000 and 110,000 km, is virtually opaque in all of our data sets; in Fig. 4 the normal optical depth exceeds 5 over much of this region. The much more transparent $\mathrm{C}$ ring and Cassini Division (the latter located between the A and B rings) are punctuated by several narrow gaps and associated ringlets. Finally, the narrow $\mathrm{F}$ ring is visible beyond the outer edge of the $\mathrm{A}$ ring, at a radius of $140,200 \mathrm{~km}$, but is unresolved at this scale.

The noise level in each of these datasets is $\sim 3 \mathrm{DN}$, as indicated by the very small signal variations seen exterior to the $\mathrm{A}$ ring and interior to the $\mathrm{C}$ ring. This is typical for VIMS occultations with good stellar pointing (see Section 6.2 below for further details.)

Two other curious, and initially unexpected, features of the VIMS occultation data are also visible in Figs. 4 and 5. First is the significant difference in transmission of the A ring between ingress and egress profiles in Fig. 5. This is now known to be due to the presence of strong self-gravity wakes in this region, which results in the apparent optical depth of the ring being dependent on longitude as well as the opening angle $\left|B_{*}\right|$ (Colwell et al. 2006; Hedman et al. 2007). Second are the overshoots in stellar flux at many of the sharpest ring edges, especially in the A ring. Modeling shows that this is due to diffraction by mm-sized particles in the rings, which contributes a forward-scattered component to the measured stellar flux immediately adjacent to ring edges in non-opaque regions (Becker et al. 2016; Harbison et al. 2019). 

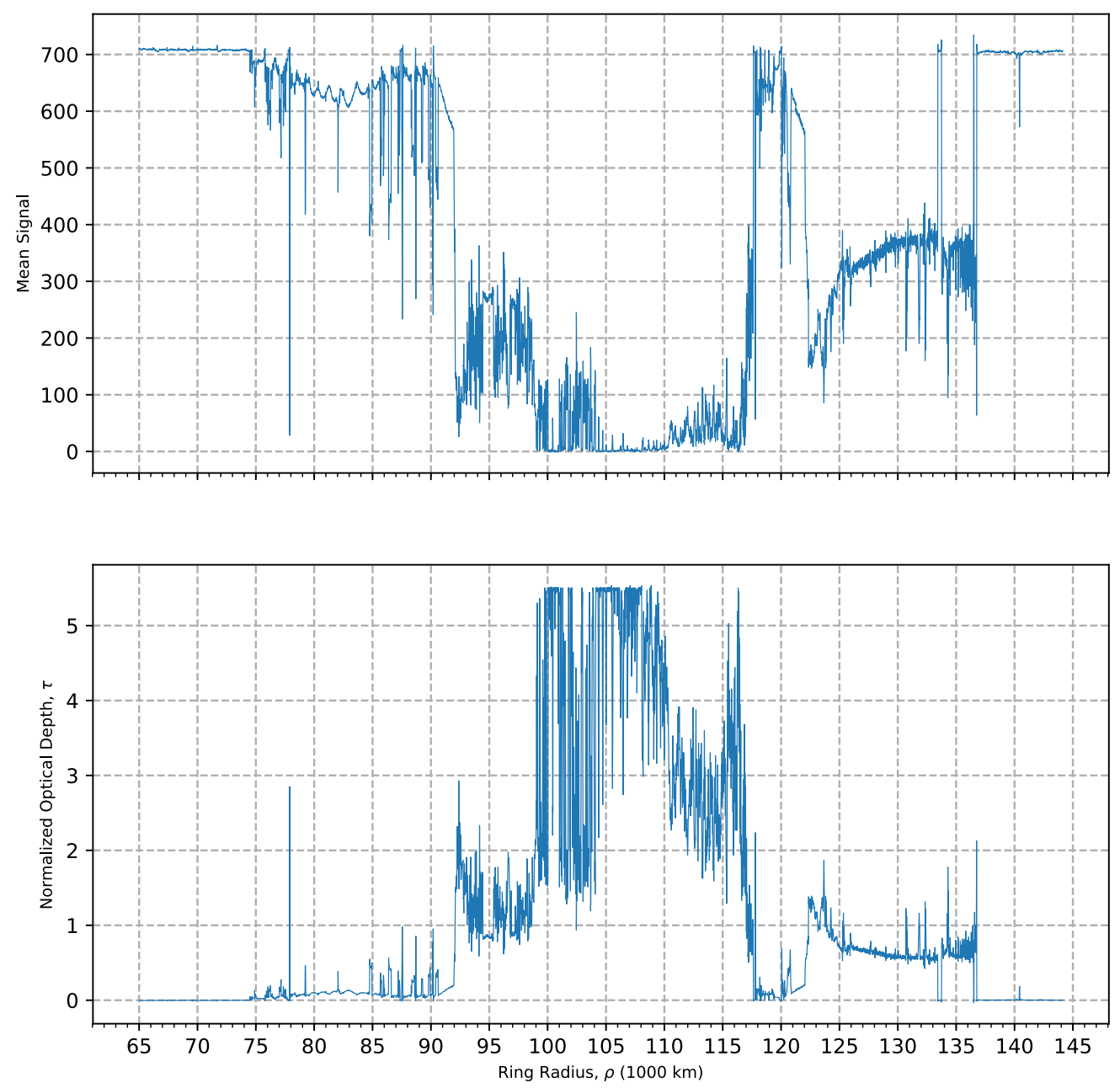

Fig. 4.- Ring profiles for the $\gamma$ Crucis occultation on rev 89. In this case, the occultation track was a complete radial ingress cut from the $\mathrm{F}$ ring to the $\mathrm{D}$ ring, crossing the $\mathrm{A}, \mathrm{B}$ and $\mathrm{C}$ rings in turn. The upper panel shows the measured light curve, in raw data numbers $(\mathrm{DN})$, while in the lower panel the data have been converted to normal optical depth. The data shown here are summed over 8 spectral channels, centered at a wavelength of $2.92 \mu \mathrm{m}$, and are plotted on a scale of radius in Saturn's equatorial plane at a radial resolution of $10 \mathrm{~km}$. The integration time was $40 \mathrm{msec}$ and the average range from Cassini to the rings was $680,000 \mathrm{~km}$, or $11.4 R_{S}$. The ring opening angle $\left|B_{*}\right|=62.35^{\circ}$. For this profile the maximum-detectable normal optical depth is 5.51, at the $3-\sigma$ level. 


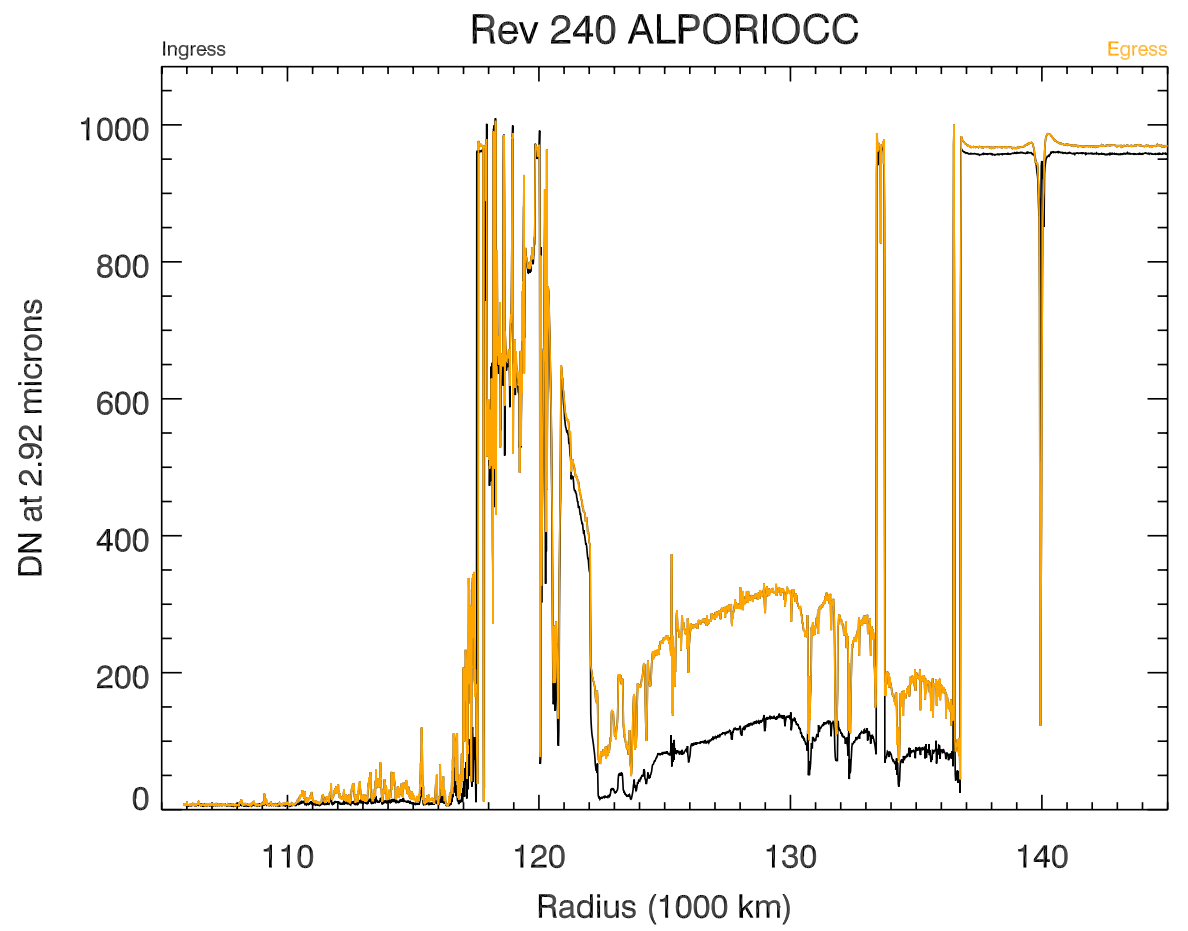

Fig. 5.- A pair of occultation profiles of the A and B rings obtained by Cassini VIMS on rev 240 for the bright, low-latitude star $\alpha$ Orionis. In this instance, the path of the star was a chord across the right ring ansa, penetrating in to a radius of $106,000 \mathrm{~km}$ in the middle $\mathrm{B}$ ring. The ingress leg of the chord is shown in black, while the egress leg is superimposed in orange/grey. As in Fig. 4, the lightcurves displayed here are summed over 8 spectral channels, centered at a wavelength of $2.92 \mu \mathrm{m}$, and the stellar transmission profiles are plotted on a scale of radius in Saturn's equatorial plane. The integration time here was $20 \mathrm{msec}$ and the average range from Cassini was $1,040,000 \mathrm{~km}$, or $17.2 R_{S}$. The ring opening angle $\left|B_{*}\right|=11.68^{\circ}$. 
Of the 190 stellar occultations listed in Table 8, only eight failed to return any useful data: four because of a failure to acquire the star, two because of a problem receiving or recording the data at the Deep Space Network station due to rain or equipment failure, and one because the onboard data-policing limits were exceeded. ${ }^{6}$ One planned observation by CIRS was subsequently used for spacecraft pointing tests. Another seven observations suffered partial losses of data due to DSN problems or data-policing.

It is more difficult to say exactly how many observations were compromised by poor pointing (i.e., instances when the star was not well-centered in a single pixel. Such cases are usually revealed by a lower-than-expected and/or variable stellar signal prior to the start of the occultation, and are generally quite obvious. Examination of the entries in Table 8 shows that $\sim 20$ data sets are flagged as being of 'poor' quality, most of which are believed to be due to pointing problems. This amounts to $10 \%$ of the total data set. In many of these cases, however, the light curves at shorter wavelengths (e.g., at $1 \mu \mathrm{m})$ are found to be less noisy than those at our standard wavelength of $2.92 \mu \mathrm{m}$, presumably because the VIMS point spread function is significantly smaller at shorter wavelengths so that less of the stellar flux fell outside the recorded pixel. Overall, between 80 and $90 \%$ of the ring occultations attempted by VIMS yielded useful data, depending on the application.

Several features of the VIMS occultation data set are worthy of note, in order to make the best scientific use of the results.

- In general stellar occultations are possible only when the spacecraft is on an inclined orbit relative to the planet's equator. As part of its overall scientific program, Cassini spent several long periods on near-equatorial orbits making observations of the icy satellites, as well as Saturn itself. These were in 2005/6, late 2007, all of 2010-2011 and in 2015. The only occultations obtained in these periods were of very low-inclination stars such as $o$ Cet, $\alpha$ Her, 30 Psc and X Oph (see Fig. 3). For these stars, all or most of regions such as the $\mathrm{A}$ and $\mathrm{B}$ rings - and even the plateaux and ringlets in the $\mathrm{C}$ ring - are effectively opaque (in the notation of Section 6.6 below, they have values of $\left.\tau_{\max } \leq 0.5\right)$. Nevertheless, these occultations can provide very useful observations of features in the D and F rings and of various low-optical depth ringlets in the Encke gap and Cassini Division.

A good example is provided by the chord occultation by o Ceti on Rev 8 , shown in Fig. 6, where $B_{*}=3.45^{\circ}$ and the unocculted stellar count rate was $990 \mathrm{DN}$ per $80 \mathrm{~ms}$ sample, or $12,400 \mathrm{DN}$ per second. Although even the A ring is virtually opaque at this highly oblique angle, we see that the Cassini Division and F ring are captured under near-optimal conditions. Note that the A ring is slightly more transparent on egress than it is on ingress, again due to self-gravity wakes (Hedman et al. 2007).

\footnotetext{
${ }^{6}$ Each observation is pre-assigned a certain data volume on Cassini's solid state recorders, and each instrument is assigned a maximum data volume for each downlink. If the latter is exceeded for any observation in the downlink due to a lower-than-expected data compression rate, then some or all data for the final observation in the downlink may be lost. This happened several times early in the mission.
} 

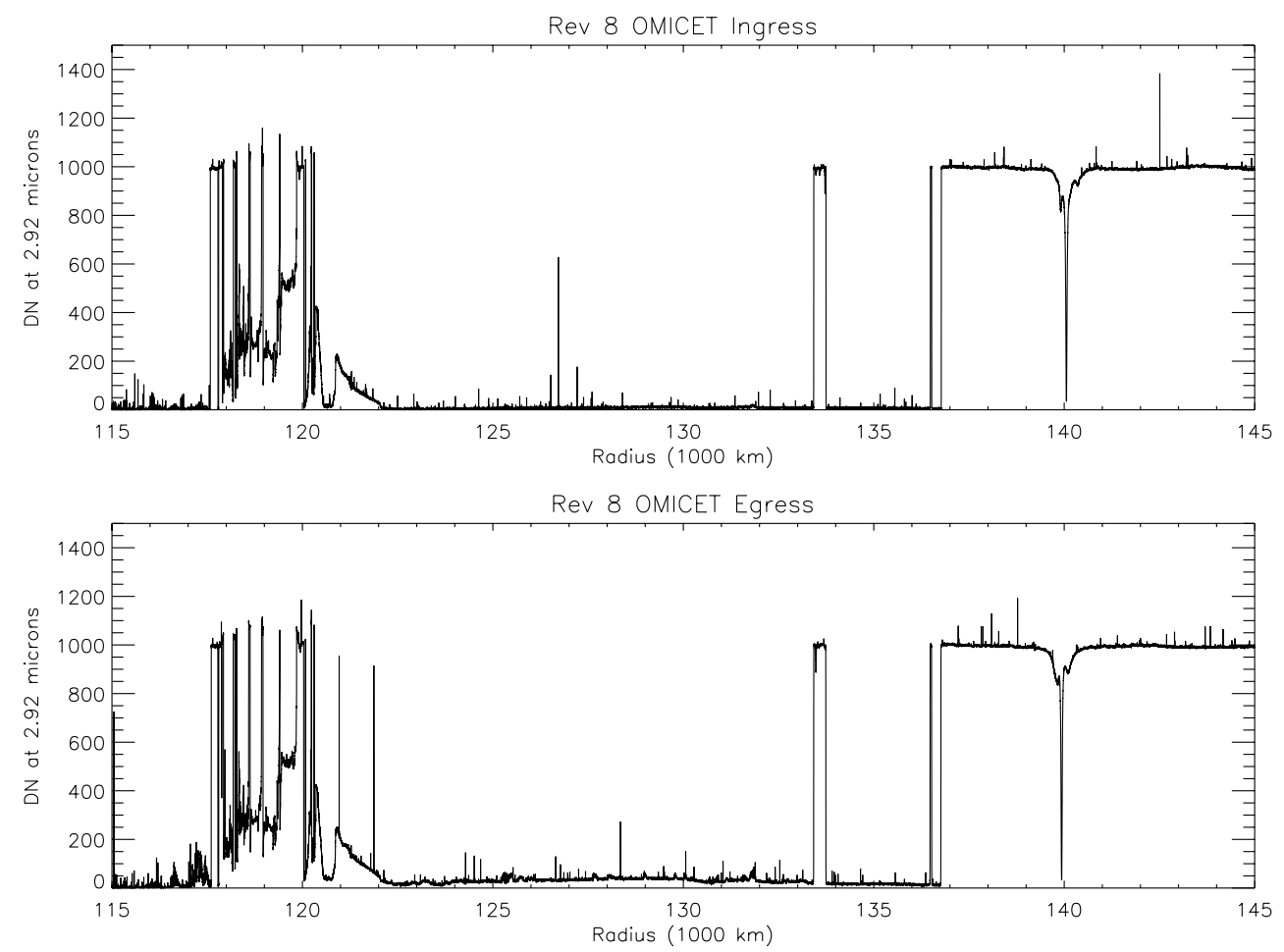

Fig. 6.- A pair of occultation profiles of the $\mathrm{F}$ and A rings and Cassini Division obtained by VIMS on rev 8 using the bright star $o$ Ceti (Mira) with a very low ring opening angle $\left|B_{*}\right|=3.45^{\circ}$. In this instance, the path of the star was a shallow chord across the left ring ansa, penetrating only to the outermost B ring. As in Figs. 4 and 5, the lightcurves displayed here are summed over 8 spectral channels, centered at a wavelength of $2.92 \mu \mathrm{m}$. In this case the ingress and egress transmission profiles are plotted separately on a scale of radius in Saturn's equatorial plane. Positive 'spikes' are due to charged particle hits on the detectors. The integration time was $80 \mathrm{msec}$ and the average range to Cassini was $1,640,000 \mathrm{~km}$, or $27.2 R_{S}$. 
- Several campaigns were conducted during the 13-year Cassini mission, each using a particular bright star in geometries that closely repeated from one orbit to the next. The most notable examples are a series of 17 radial occultations by $\gamma$ Cru on revs $71-102$, a set of seven radial or chord occultations by $\alpha$ Sco on revs 237-245, a series of eleven mostly chord occultations by $\alpha$ Ori on revs $240-277$ and a second series of nine radial $\gamma$ Cru occultations on revs 245-292. Other shorter series of similar occultations involved the stars R Leo, R Cas, R Lyr, W Hya, RS Cnc and $\mathrm{L}^{2}$ Pup. We have found these series to be particularly useful in understanding longitudinally-variable features such as spiral waves, eccentric ringlets, noncircular gap edges, etc., partly because of their uniform sensitivities and constant value of $B_{*}$ and partly because of their relatively dense temporal sampling. See, for example, Hedman and Nicholson (2013, 2014).

- In most instances, the radial resolution of VIMS stellar occultations is set by the instrument's integration time, rather than by the Fresnel zone and/or the projected stellar diameter. As a consequence, the resolution frequently improves with increasing distance to the planet, because the spacecraft velocity near the apoapse of its orbit is reduced compared to the value near periapse. This means that some of our highestresolution observations have been done at distances of 20-30 $R_{S}$, notably with $o$ Ceti on revs 8-12 and $\mathrm{L}^{2}$ Pup on revs 198-206. In several cases, these very slow occultations have permitted the measurement of the stars' angular diameters at multiple nearinfrared wavelengths - or even their 2D shape in the case of $o$ Ceti - using sharp ring edges (Stewart et al. 2016a,b).

In Fig. 7 we compare the radial and azimuthal sampling intervals for all VIMS occultations by the A ring with the corresponding Fresnel zone diameters and projected stellar diameters. In this figure, colors distinguish different stars. For example, the very large brown circle in the lower panel represents the W Hya occultation on rev 236, with a Fresnel zone diameter of $130 \mathrm{~m}$ and a projected stellar diameter of $\sim 550 \mathrm{~m}$, while the pair of large red circles indicate the ingress and egress cuts of the $\alpha$ Sco occultations on revs 237 and 238, for which the stellar diameter was $\sim 300 \mathrm{~m}$. The azimuthal sampling is calculated relative to the local co-moving (i.e., keplerian) frame, which accounts for the preponderance of negative sampling intervals. Negative and positive radial sampling intervals correspond to ingress and egress occultations, respectively. It can be seen here that the typical (sampling) resolution of the VIMS occultations is $\sim 250 \mathrm{~m}$ in the radial direction and $\sim 750 \mathrm{~m}$ in the azimuthal direction, though wide variations occur due to the diversity of occultation geometries. There are no VIMS ring occultations for which both the radial and azimuthal velocities are simultaneously close to zero (these have been referred to as 'tracking occultations', as the star's motion briefly matches that of the ring particles), but there are several events for which the azimuthal velocity is close to zero for some part of the observation. These include $\alpha$ Sco on rev 13, R Leo on revs 68 and $75, \beta$ Gru on rev 78 and R Dor on revs 186 and 188.

Also indicated in this figure is the typical orientation of the self-gravity wakes, canted 
in a trailing direction by $\sim 20^{\circ}$ from the azimuthal direction. A point lying on or near the diagonal lines at 4 o'clock or 10 o'clock means that the star moved in a direction relative to the ring material that was approximately parallel to the self-gravity wakes, whereas a point near the 1 o'clock or 7 o'clock lines means that the star moved in a direction approximately perpendicular to the wakes. The latter are more likely to be able to resolve the widths of individual wakes, though very few VIMS occultations have sufficient resolution for such studies.

- Binary stars can yield particularly interesting — if sometimes confusing — information on very small-scale azimuthal variations in ring structure. Among the VIMS stars, many of whom are known binaries, only $\alpha$ Centauri has a companion bright enough to produce obvious secondary features in the occultation light curves, as seen in the upper panel of Fig. 8. Note the double-step at each sharp edge, due to the separate occultations of the two components of the binary system. For this particular geometry and time, their projected radial separation was $\sim 15 \mathrm{~km}$, or $\sim 3^{\prime \prime}$ at the Cassini-Saturn distance of $1,055,000 \mathrm{~km}$, or $17.5 R_{S}$. Away from these edges, the binary nature of the star is much less obvious. VIMS has observed 3 occultations by this system, on revs 66, 105 and 247, but no study of any possible azimuthal variations has been published to date.

- Because of their greatly differing sensitivities to hot and cool stars, UVIS and VIMS cannot generally observe occultations by the same star, or stellar system. Exceptions include five occultations of $\alpha$ Lyr (Vega), a bright A0 star, and six of $\alpha$ CMa (Sirius). The latter system has a fairly bright A-type primary (observable by VIMS) and a very hot white dwarf secondary (observable by UVIS). $\alpha$ Sco is also a binary where component A is visible to VIMS and component B is visible to UVIS. In at least one occultation by this star observed by both instruments it was possible to confirm the reality of a $500 \mathrm{~m}$-wide clump of some sort in the $\mathrm{F}$ ring that occulted both stars (Esposito et al. 2008). Comparisons of the optical depths measured simultaneously by both instruments in occultations by these stars have also made it possible to draw useful conclusions about the small end of the ring particle size distribution in some regions (Colwell et al. 2014; Jerousek et al. 2016), avoiding the complications introduced by the ubiquitous self-gravity wakes.

- As noted in Section 1, Cassini's other infrared instrument, CIRS, is able to observe occultations by two very bright mid-IR objects: CW Leonis (also known as IRC+10216) and $\eta$ Carinae. Both are stellar objects in the brief post-main-sequence phases of their evolution and are shrouded in thick dust shells, and both can also be observed by VIMS, albeit only at longer wavelengths. Occultations were observed successfully for CW Leo on revs 31, 70 and 74 and for $\eta$ Car on revs 194, 250 and 269, but no comparative studies have so far been published. An example of a VIMS occultation of $\eta$ Car is illustrated in the lower panel of Fig. 8, where we again show the outermost part of the A ring. The extended nature of the source is apparent from the more muted ring edges compared with the profile in the upper panel of this figure. For this particular observation the projected diameter appears to be $\sim 7 \mathrm{~km}$, or $\sim 2^{\prime \prime}$ at 

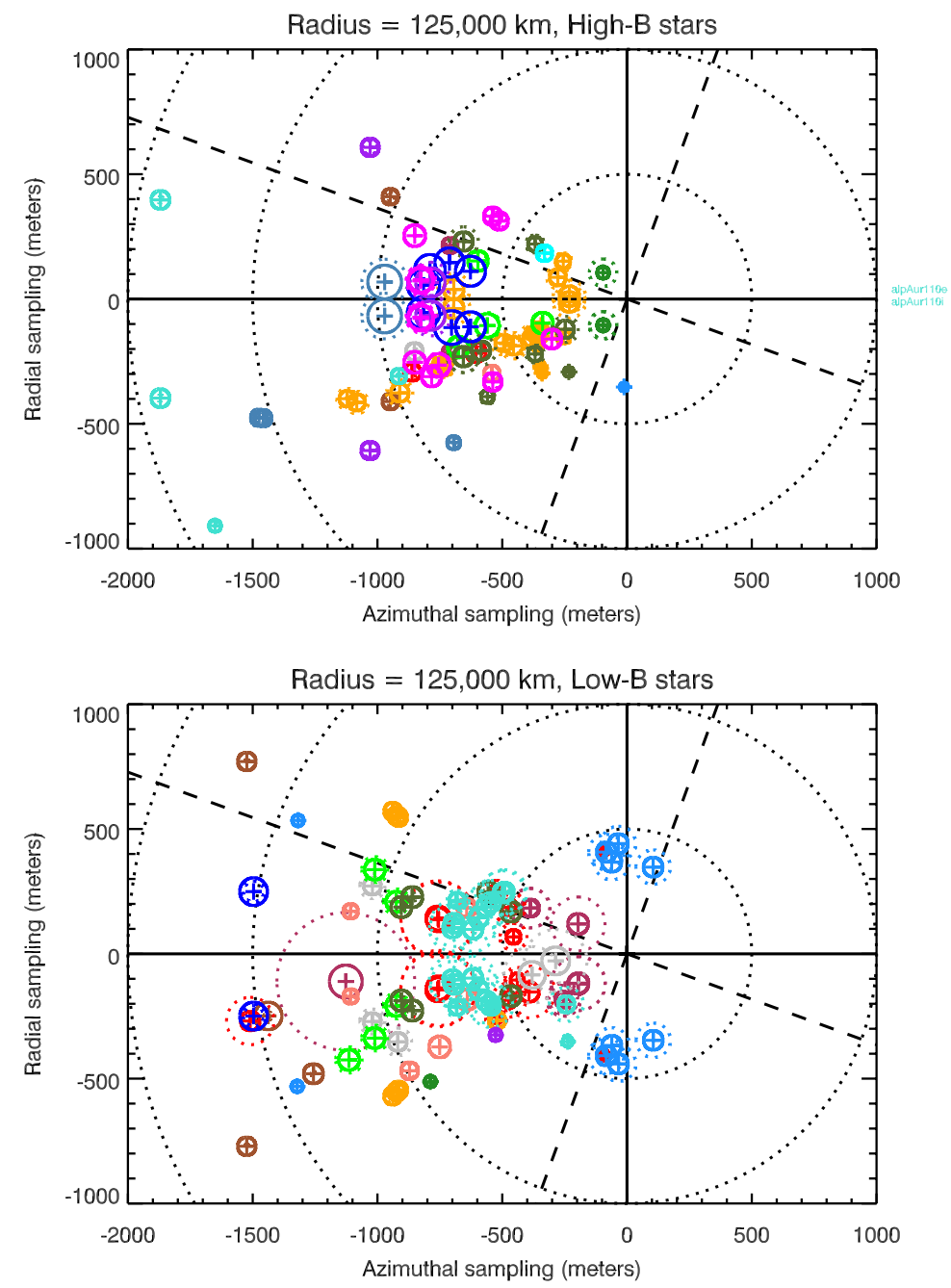

Fig. 7.- Scatter plot showing the radial and azimuthal sampling intervals for all VIMS stellar occultations which crossed the central A ring, evaluated at a radius of $125,000 \mathrm{~km}$. Negative radial sampling intervals correspond to ingress occultation segments and positive radial intervals to egress segments. The azimuthal sampling is calculated relative to the local co-moving (i.e., keplerian) frame. Different colors indicate different stars. The upper panel shows data for high-inclination stars $\left(\left|B_{*}\right|>40^{\circ}\right)$, while the lower panel shows data for low-inclination stars. For each star, a solid circle indicates the diameter of the Fresnel zone $\sqrt{2 \lambda D}$, while a dashed circle indicates the projected stellar diameter at the distance of the rings, as given in Table 10 in the Appendix. Diagonal lines denote the typical orientation of the self-gravity wakes in the A ring (see text). 

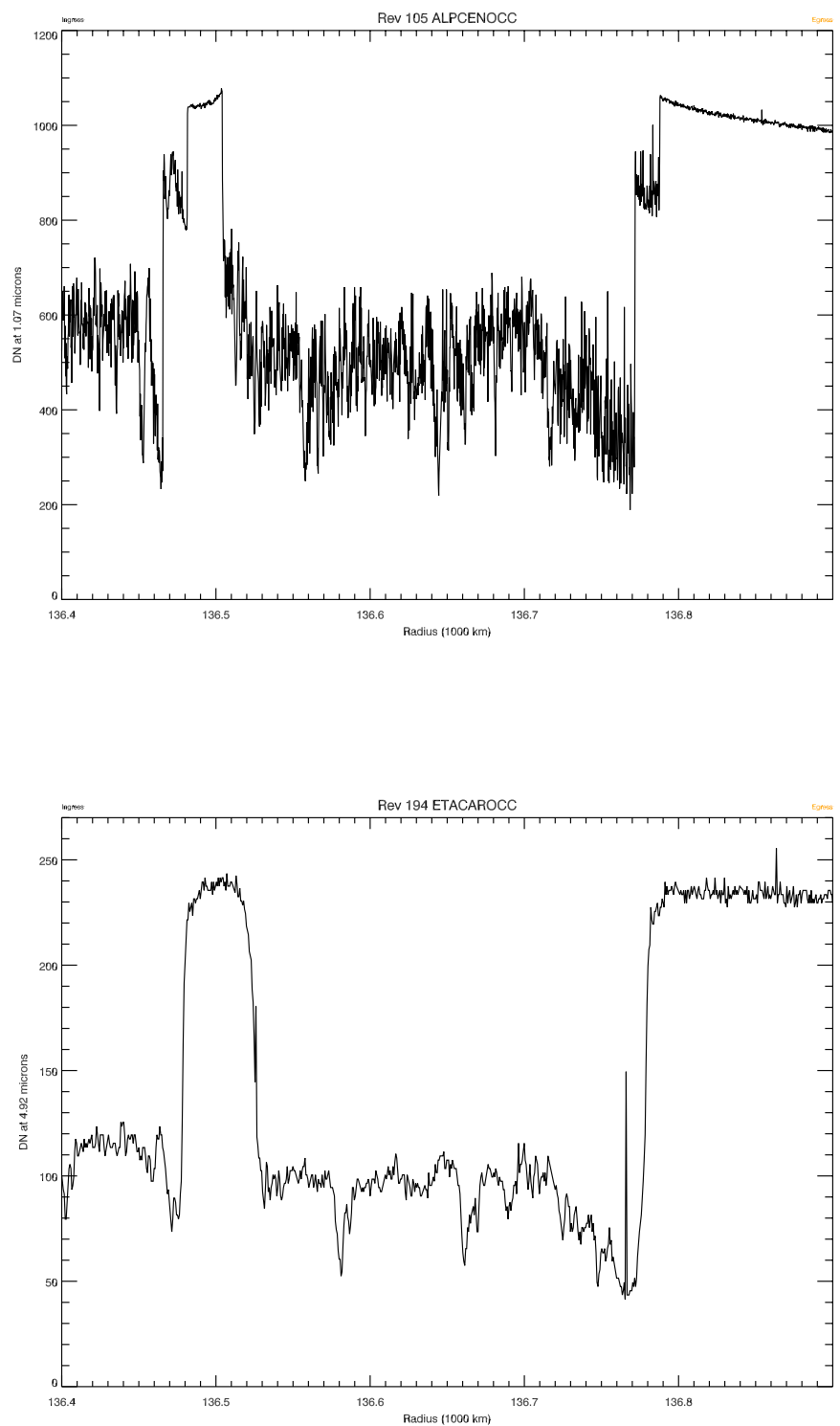

Fig. 8.- (Upper panel) An occultation profile of the outermost part of the A ring obtained by VIMS on rev 105 for the visual binary star $\alpha$ Centauri. As in Fig. 4, the lightcurve is summed over 8 spectral channels, but in this case centered at a wavelength of $1.07 \mu \mathrm{m}$. The integration time was $40 \mathrm{msec}$ and the ring opening angle $\left|B_{*}\right|=67.30^{\circ}$. (Lower panel) An occultation profile of the same part of the A ring obtained by VIMS on rev 194 for the peculiar, pre-supernova object $\eta$ Carinae. Again the lightcurve is summed over 8 spectral channels, but in this case centered at a wavelength of $4.92 \mu \mathrm{m}$. The integration time was $80 \mathrm{msec}$ and the ring opening angle $\left|B_{*}\right|=62.47^{\circ}$. In both panels, the $500 \mathrm{~km}$ segment of data plotted shows the outer edge of the A ring at $\sim 136,770 \mathrm{~km}$ and the $35 \mathrm{~km}$-wide Keeler gap at a mean radius of $\sim 136,480 \mathrm{~km}$, at the full sampling resolution of the data. 
the Cassini-Saturn distance of $675,000 \mathrm{~km}$, or $11.2 R_{S}$. This strongly suggests that we are sensing the warm dust shell rather than the stellar photosphere. Joint studies of the CIRS and VIMS data at multiple wavelengths might throw more light on the shell structure of this unique object, as well as probing ring optical depths in the mid-infrared.

\section{Geometric calibration}

\subsection{Basic occultation geometry}

Our algorithm for converting the time of each sample as recorded at the spacecraft into the radius and longitude at which the ray from the star pierced the ring plane is based on that described by French et al. (1993). Of the several alternate schemes described therein we have chosen to do the calculation in a saturnicentric reference frame as this is both conceptually and operationally simpler and also presumably matches the reference frame used by the Cassini project to calculate the spacecraft trajectory. In this calculation the key variables, apart from the spacecraft trajectory, are the apparent direction towards the star and the planet's pole vector. Cassini's trajectory is available in the form of SPK files and is accessed via routines from the NAIF SPICE library (Acton 1996).

To obtain the stellar position, we start with the heliocentric position at J2000 given in the Hipparcos catalog (fortunately almost all of our VIMS stars appear here) and then apply proper motion corrections and parallax to obtain the stellar position as seen from Saturn at the time of the occultation. We then correct this position for stellar aberration, using the heliocentric velocity of Saturn, to obtain the apparent direction to the star as seen from an observer moving with Saturn. (In a few cases where Hipparcos positions are not available, we use the position and parallax given by the SIMBAD web site.) At a typical range from Saturn to Cassini of $10 R_{S}$, or $600,000 \mathrm{~km}$, an error of 10 mas in the stellar position maps into an error in the calculated ring plane position of $\sim 30 \mathrm{~m}$. For even the most distant occultations, at ranges of about $3 \times 10^{6} \mathrm{~km}$, the likely errors due to this source of error in the ring-intercept position are at most $150 \mathrm{~m}$. However, neglect of parallax or stellar aberration can easily lead to much larger errors of $20-90 \mathrm{~km}$ at these same distances. ${ }^{7}$

For Saturn's pole vector, which may be assumed to be perpendicular to the ring plane for all practical purposes (Nicholson et al. 1990), we use the right ascension and declination specified in the Cassini PCK files, derived from the precessing pole model of French et al. (1993), as updated by Jacobson et al. (2011). The current uncertainty in the pole is $\sim 0.1$ arcsec, due to uncertainties in the precession model (French et al. 2017), which can map into an error in the calculated ring-intercept position of order $50 \mathrm{~m}$ for low-inclination stars but much less when $\left|B_{*}\right|$ is large.

\footnotetext{
${ }^{7}$ An alternative approach which avoids aberration corrections is to carry out the geometric calculation in a heliocentric reference frame, but this necessitates an iterative procedure to compute the light travel time from the rings to the observer.
} 
An important issue, and probably the limiting factor in the absolute accuracy of the calculated ring positions, is the uncertainty in the spacecraft trajectory. Calculations using 'predict' trajectories are frequently in error by several km. Generally, we can correct these first-order errors by applying a time offset to the predicted trajectory, typically of order $1 \mathrm{sec}$ but occasionally amounting to several seconds. Subsequent calculations using reconstructed trajectories supplied by the Cassini Navigation team are usually accurate to $\leq 1 \mathrm{~km}$, based on the observed radii of quasi-circular features in the rings. For the latter we use the list provided by French et al. (1993), as updated by French et al. (2017). Eventually, it is hoped that a single reconstructed Cassini trajectory will be available, consistent with the best estimates for the pole position, planet and satellite masses, and Saturn's zonal gravity harmonics, but this has not yet been achieved. In the meantime, it is possible to use the known radii of quasi-circular ring features to derive small trajectory corrections for the neighborhood of each occultation, reducing the radius errors to $\sim 150 \mathrm{~m}$ (French et al. 2017).

Comparisons of our calculated ring radii with independent computations by R.G. French using his very well-tested heliocentric algorithm show agreement at the $1 \mathrm{~m}$ level, if we use the same trajectory, star catalog and pole vector. For completeness, we provide in Appendix A a more detailed description of our geometric calculations.

Figure 9 illustrates the geometry for a typical chord occultation, that of $\alpha$ Sco on rev 13. We have found it useful to be able to view the occultation track in two ways, in vertical projection from above Saturn's north pole and in a perspective view as seen from the star, both of which are illustrated here. The former best shows the coverage with respect to the rings, including the azimuthal and radial motion of the star as well as the minimum radius probed and the location of Saturn's shadow. The perspective view, on the other hand, shows the track's location relative to the ring ansa, and whether or not the star came close to, or was occulted by, the planet. Note that in this case the star came very close to being occulted by the planet's south pole during the ingress occultation of the $\mathrm{B}$ ring.

\subsection{Additional comments}

A few peculiarities of stellar occultations are worth noting. First and foremost, it is evident that any occultation by a particular star is always observed at the same ring opening angle, regardless of the spacecraft trajectory. This angle is equal to the absolute value of the star's planetocentric latitude $B_{*}$, which in turn is determined by the star's right ascension and declination and the planet's pole direction, which we assume to be fixed. In this paper, we will treat the planetocentric latitude of the star and the ring opening angle of an occultation as interchangeable quantities, except for the sign attached to the former, and denote this important quantity by $B_{*}$ in Tables 1,4 and 5 . However, the astute reader may notice that $B_{*}$ is not in general equal to the saturnicentric latitude of the spacecraft, which is measured from the center of the planet. During a typical occultation, the latitude and range of the spacecraft change continuously, while the angle between the stellar line-of-sight 

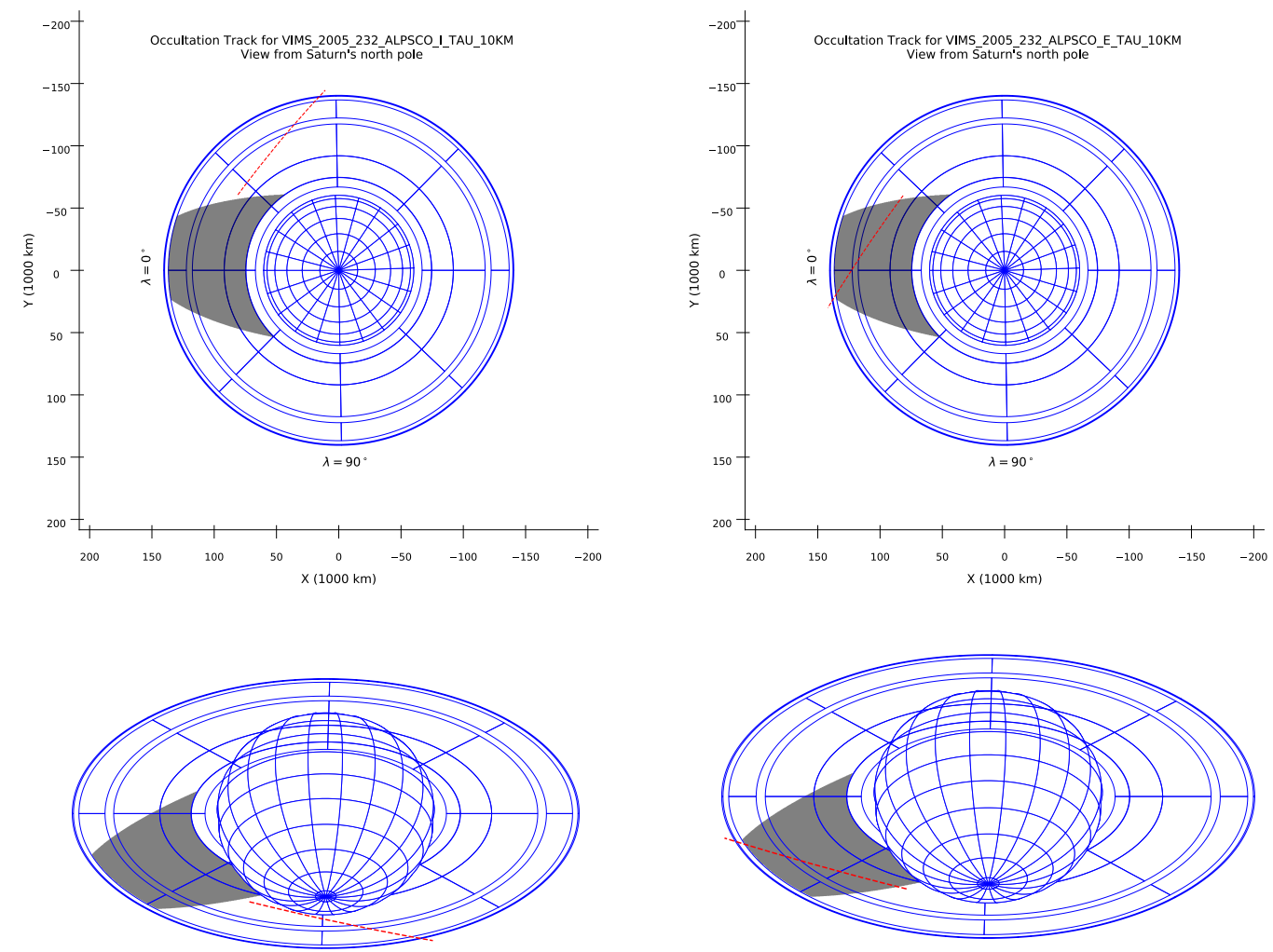

Ring Elevation (deg): -32.1

Ring Elevation (deg): -32.1

Fig. 9.- Two views of the geometry for the chord occultation of $\alpha$ Sco on rev 13. The upper two panels show the track of the star across the rings as seen from a vantage point above Saturn's north pole, for the ingress and egress segments of the occultation. Circles denote the inner and outer radii of the $\mathrm{F}, \mathrm{A}, \mathrm{B}, \mathrm{C}$ and $\mathrm{D}$ rings. The $+\mathrm{X}$-axis of the coordinate system points towards a longitude of $0^{\circ}$, and the grey region indicates the shadow cast by Saturn on the rings. The lower two panels show perspective views of the occultation track, as seen from the star, for ingress and egress. These plots show the apparent path of Cassini behind the rings, a seen from an infinite distance. Saturn's north pole points upwards. 
and the ring plane remains fixed. For occultations that occur near periapse this can make it difficult to visualize the apparent path of the star behind the rings, without recourse to a movie or at least a sequence of diagrams. It is for this reason that we prefer instead to illustrate the occultation geometry by plotting the track of Cassini as seen from an infinitelydistant observer in the direction of the occulted star, as shown in the lower panels of Fig. 9. The fundamental geometry of the event is unchanged, due to the reversibility of light rays, but from this perspective the aspect of the planet and rings remain fixed throughout the event and a single diagram can accurately represent the geometry. (We have borrowed this unconventional 'trick' from the practitioners of spacecraft radio occultations, who almost always plot the track of the spacecraft as seen from the Earth, rather than vice versa.)

Similarly, the inertial longitude of the vector from the spacecraft to the star, denoted by $\lambda_{*}$ below, is always the same. (Technically, $B_{*}$ and $\lambda_{*}$ vary slightly between occultations due to changing parallax and aberration, but this is important only for precise geometric reconstruction of the target point in the rings.) Combining this with the (time varying) longitude of the occultation point in the rings, $\lambda$, we find that the angle in the ring plane between the stellar line-of-sight and the local radial direction is given by $\phi=\lambda-\lambda_{*}$. In diagrams such as those in the lower panels of Fig. $9, \phi=0$ at the point on the rings closest to the star (the top, in this case), $\phi=90^{\circ}$ at the right ansa and $\phi=270^{\circ}$ at the left ansa.

The two quantities $B_{*}$ and $\phi$ largely determine how the stellar signal is attenuated by the ring at any given point in the occultation. In particular, the transmission of a homogeneous, flat ring is given by

$$
T=e^{-\tau / \sin B_{*}},
$$

where $\tau$ is the normal optical depth, while the transmission of a ring with self-gravity wakes is determined by a more complex combination of $B_{*}$ and $\phi$ (Colwell et al. 2006, 2007; Hedman et al. 2007). The wake geometry is illustrated in Fig. 5 of Hedman et al. (2007). Fig. 10 shows the distribution of $\left|B_{*}\right|$ and $\phi$ for all VIMS ring occultations, evaluated in the central A ring. The data span the range from $1.1^{\circ} \leq\left|B_{*}\right| \leq 74.2^{\circ}$, and the full range of $\phi$, with some preference for the quadrant centered on $\phi \sim 110^{\circ}$. The latter reflects the fact that the majority of VIMS observations were of ingress occultations, which typically cross the left ansa of the rings, as seen from the spacecraft, or the right ansa as seen from the star. Vertical dashed lines at $\phi=70^{\circ}$ and $250^{\circ}$ in Fig. 10 show the longitudes for which the self-gravity wakes are viewed end-on, and the ring's transparency is a maximum, while dotted lines denote the orthogonal directions where the rings are most opaque.

The quantities $B_{*}$ and $\phi$ also determine the sensitivity of occultation data to vertical structure in the rings (Gresh et al. 1986; Nicholson et al. 1990; Jerousek et al. 2011). The apparent radial displacement of a ring feature displaced vertically from the ring plane by $z$ is given by $\delta r=-z \cos \phi / \tan B_{*}$. For example, Nicholson and Hedman (2016) use this expression to model the appearance of a gap in the bending wave driven in the inner $\mathrm{C}$ ring at the Titan nodal resonance, using a large suite of VIMS occultation data. 


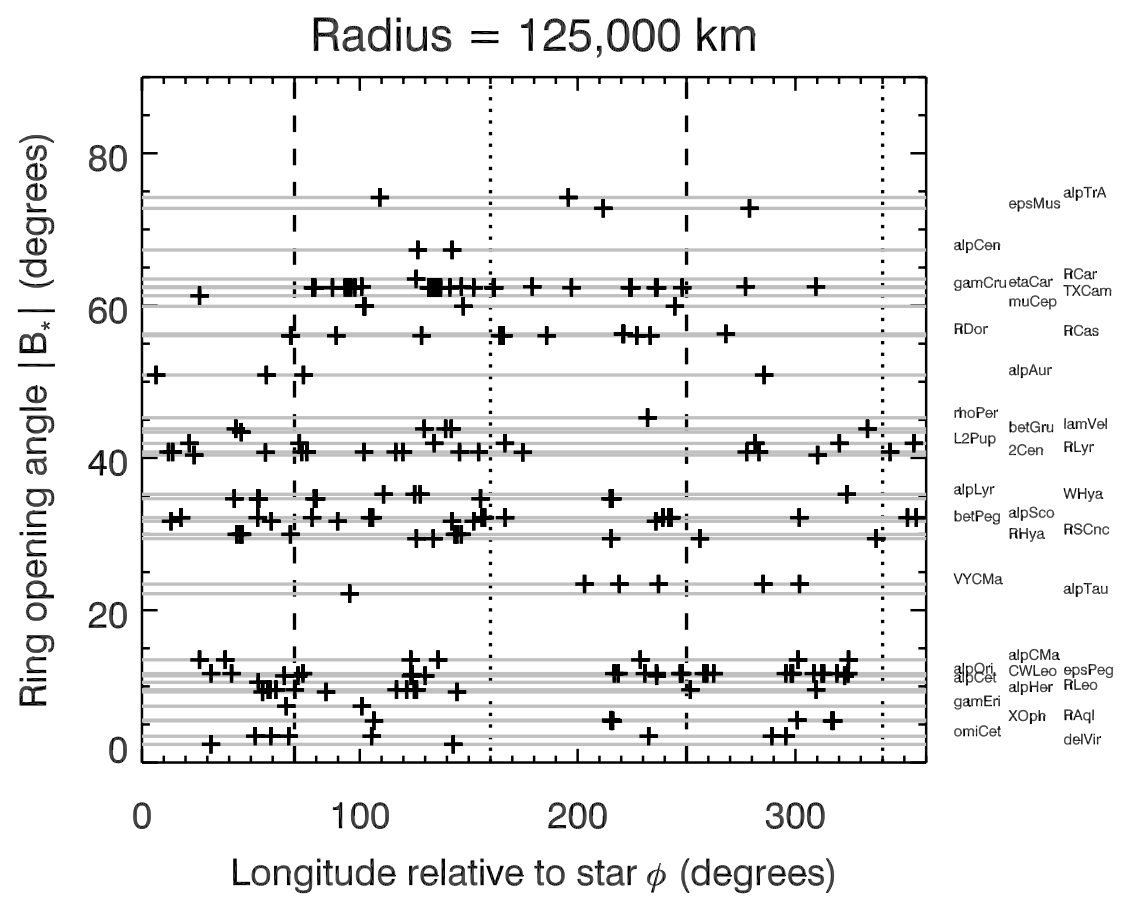

Fig. 10.- Scatter plot showing the distribution of ring opening angle, $\left|B_{*}\right|$ and occultation longitude, $\phi$ relative to the direction towards the star for all VIMS ring stellar occultations which crossed the central A ring, evaluated at a radius of 125,000 km. For each star, a faint horizontal line indicates the value of $B_{*}$, as given in Table 1 . Dashed lines at $70^{\circ}$ and $250^{\circ}$ denote the longitudes for which the self-gravity wakes are viewed end-on, and the ring's transparency is a maximum, while dotted lines denote the orthogonal directions. 


\section{Photometric calibration}

\subsection{Calibration procedure}

In standard 'pipeline' processing, VIMS cube data are background-subtracted, processed to identify and remove bright pixels due to charged particle hits, and then converted to I/F, a standard measure of reflectivity for solar system objects. The final step involves converting the raw data numbers (DN) to incident radiance on the instrument, using a calibration function which has been steadily updated during the mission, and then to reflectivity using a reference solar spectrum (McCord et al. 2004; Clark et al. 2012, 2018). For stellar occultation observations, only the background-subtraction step is applied, because the remaining steps are either impossible (identifying charged-particle hits involves acquiring a $2 \mathrm{D}$ image) or inappropriate (converting DNs to I/F). Instead, our goal is to reduce the observed stellar signal to transmission through the rings, while minimizing extraneous sources of signal variations. To this end, we have adopted the following procedure:

- The original measured instrumental backgrounds are restored to the data (i.e., we undo the onboard line-by-line background subtraction) to reconstruct the raw measured signal $S(\lambda, t)$.

- All of the measured background spectra for a given occultation are combined to produce a single average background spectrum, $\bar{B}(\lambda)$. This has the effect of removing any random charged-particle hits on the individual backgrounds, which can lead to incorrect offsets in sets of 64 contiguous measurements.

- This average background is subtracted from all of the raw stellar measurements: $F(\lambda, t)=S(\lambda, t)-\bar{B}(\lambda)$.

- The next step is to normalize the stellar flux, in order to calculate the ring's transmission $T$ as a function of radius and wavelength. At each wavelength, the average unocculted signal is determined by forming the median of the background-corrected signal in the region exterior to the $\mathrm{F}$ ring, between radii of 143,000 and 145,000 km, denoted by $F_{0}(\lambda)$. (If these data are unavailable, we use either the region between the $\mathrm{A}$ and $\mathrm{F}$ rings, known as the Roche Division, or that interior to the $\mathrm{C}$ ring, at radii less than $74,400 \mathrm{~km}$. Although the latter two regions are occupied by tenuous ring material, their optical depths are so low $\left(\sim 10^{-3}\right)$ that they are virtually undetectable in the VIMS occultation data.) We do not make use of the various narrow gaps in the rings for this purpose, as the signal levels here frequently exceed $F_{0}$ due to forward-scattering by nearby ring material, as seen in Fig. 5 .

- The background-corrected signal is then divided by the mean unocculted stellar signal to yield an estimate of the ring transmission, $T(\lambda, t)=F(\lambda, t) / F_{0}(\lambda)$. Ideally, we should first correct both $F(\lambda, t)$ and $F_{0}(\lambda)$ for any nonstellar contribution to the measured flux, e.g., reflected or transmitted sunlight from the rings, or scattered light from the planet, but in practice we do not have a direct way to measure this, short 
of replicating the occultation geometry at a time when the star is not present. An alternative is to choose a wavelength where the albedo of the icy rings is very low, and any reflected light is negligible. We have found that it is sufficient in almost all cases to use the measured flux in (summed) IR channel 15, corresponding to a mean wavelength of $2.92 \mu \mathrm{m}$. At this wavelength, the water ice that dominates the rings' reflectance spectrum is almost completely black and the rings' contribution to the measured flux can be neglected (Hedman et al. 2013).

- Finally, the transmission is converted to normal optical depth, $\tau_{\mathrm{n}}$, using the standard expression $\tau_{\mathrm{n}}=-\left|\sin B_{*}\right| \ln (T)$, where $B_{*}$ is the saturnicentric latitude of the star (see Section 5).

Researchers working with VIMS spectra should be aware that the VIMS wavelengths shifted throughout the mission (Clark et al. 2018). Shifts amounted to about $24 \mathrm{~nm}(=1.5$ VIMS channels) during the Jupiter fly-by and about $10 \mathrm{~nm}(60 \%$ of a channel) during the Saturn orbital tour. Wavelength shifts do not impact the derivation of transmission spectra during any given occultation, but comparison of spectral structure between occultations should be done with the correct wavelengths at the time of each occultation.

\subsection{Caveats and limitations}

In following the above calibration procedure, we are making several assumptions, some of which are not always true.

\subsubsection{Instrumental background variations}

In a few cases, the measured instrumental background levels are either unusually noisy or show systematic trends over time. These are identified by the quality code 'B' in Table 8 , and are flagged by a Note in the descriptive text files delivered to the PDS. Most such variations are quite gradual, monotonic and typically no greater than 5-10 DN. In such cases, if so desired, it should be possible to improve the photometric calibration by subtracting a polynomial or spline fit to the background signal rather than the average value. A unique case is the o Cet occultation on rev 135, where the background at $2.92 \mu \mathrm{m}$ varies irregularly by several tens of DN on timescales of a few minutes three or four times during the $2.5 \mathrm{hr}$ duration of the occultation.

\subsubsection{Stellar flux variations}

A more serious assumption is that the unocculted stellar flux is constant throughout the period of the occultation. As noted in Section 2 above, this may not be true if the initial star-acquistion is not successful, especially if the spacecraft pointing results in the 
stellar flux being divided between two pixels. One can generally identify such situations quite easily by examining the stability of the stellar flux in the regions outside the rings, but there is no objective way in which it can be corrected after the fact. ${ }^{8}$ The data from such occultations are still quite useful, (e.g., for measuring the times of sharp-edged features), but they should not be used for quantitative measurements of transmission or optical depth. Such cases are identified by the code ' $\mathrm{V}$ ' in Table 8 , and flagged by a Note in the descriptive text files delivered to the PDS, whenever these variations exceed $2 \%$. As noted above, a total of $\sim 20$ occultations in Table 8 are classified as Quality Code 3, mostly due to serious pointing problems, but over $100(55 \%)$ show some variation in the unocculted stellar flux. Fortunately this is typically at the 1-2\% level and is likely to cause problems only for applications where absolute optical depths are critical.

\subsubsection{Nonstellar background flux}

Finally, we are ignoring any contribution to the measured flux $F$ by reflected sunlight from the rings, or scattered light from Saturn. As noted above, we have attempted to minimize the former by only using data from summed channel 15 , corresponding to a mean wavelength of $2.92 \mu \mathrm{m}$ where the rings are very dark. In principle, it might be possible to improve on this procedure by combining the data at $2.92 \mu \mathrm{m}$ with that from another channel where the ring albedo is high, and solving for the ring and stellar contributions individually. However, this technique would introduce additional photon noise due to the ring signal (which is frequently larger than the stellar signal, even for bright stars), as well as systematic errors due to possible variations in the shape of the ring spectrum with viewing geometry. Photometric models that include such variations have only recently been published (Ciarniello et al. 2018) and we must leave this to future studies. As for possible contributions from Saturn, the VIMS-IR channel was found to be relatively immune to off-axis scattered light, and we have not observed any situations where scattered light from Saturn appears to affect the ring occultation data, even when the star is quite close to the sunlit limb.

The potential effects of reflected light in the VIMS occultation data are illustrated in Fig. 11, where we present lightcurves for the ingress occultation of $\gamma$ Crucis on rev 255 at three different wavelengths: $1.07,2.25$ and $2.92 \mu \mathrm{m}$. In this event, the star was initially seen through the sunlit rings, at a phase angle of $\sim 65^{\circ}$, but mid-way through the occultation the star passed into the planet's shadow on the rings, remaining within the shadow for the remainder of the observation. Before the occultation begins, the stellar signals are roughly 600,430 and $250 \mathrm{DN}$, respectively, per integration. At $1.07 \mu \mathrm{m}$ the rings

\footnotetext{
${ }^{8}$ In some cases, it is tempting simply to fit a low-order polynomial to the measured flux interior and exterior to the rings and in the several empty gaps in the A and C rings, as well as the Cassini Division, but this is difficult to justify quantitatively in the absence of a detailed model of the sub-pixel pointing variations during the occultation. We have elected not to attempt such corrections, except in very limited cases, preferring to leave this to the judgement of future users of the data.
} 
are highly-reflective and the signal from the A or B rings falling within 1 VIMS pixel is greater than that of the unocculted star. The measured signal at $1.07 \mu \mathrm{m}$ thus increases as the star passes behind the outer edge of the A ring at $136,770 \mathrm{~km}$, while that at $2.92 \mu \mathrm{m}$, where the rings are very faint, decreases by $\sim 50 \%$, as expected. At $2.25 \mu \mathrm{m}$ the situation is intermediate, with the rings' albedo being less than that at $1.07 \mu \mathrm{m}$ but much higher than at $2.92 \mu \mathrm{m}$, and the signal drops by a smaller fraction on entry into the A ring. Entering the Cassini Division at $122,000 \mathrm{~km}$, which is much less opaque than the A ring, and also much less bright, the observed signal at $1.07 \mu \mathrm{m}$ decreases back to a level similar to that of the unocculted star, while the signals at $2.25 \mu \mathrm{m}$ and $2.92 \mu \mathrm{m}$ increase significantly. Upon entering the $\mathrm{B}$ ring at $117,550 \mathrm{~km}$, which is both brighter and more opaque than the A ring, the signal increases to its maximum level of $\sim 1200 \mathrm{DN}$ at $1.07 \mu \mathrm{m}$, decreases modestly at $2.25 \mu \mathrm{m}$ and almost disappears at $2.92 \mu \mathrm{m}$. At a a radius of $\sim 108,000 \mathrm{~km}$, however, the star enters Saturn's shadow and the contribution of reflected sunlight to the measured count rates rapidly decreases to zero. From this point onwards, the signal in all three channels is purely stellar, and the three lightcurves look almost identical across the inner B ring and the $\mathrm{C}$ ring.

Experience suggests that any nonstellar contribution to the measured flux at $2.92 \mu \mathrm{m}$ can generally be neglected, especially on the unlit side of the rings. But as a check, we have found it prudent to monitor the residual signal in the most opaque part of the $\mathrm{B}$ ring, i.e., in parts of the B2 and B3 regions, where the normal optical depth is found to exceed 5.5 (see Fig. 16 below) and the predicted stellar signal is $\leq 3 \mathrm{DN}$, even for the brightest stars. In some situations, notably when the instrument is looking at the sunlit side of the rings at a low phase angle, we have seen evidence for ring contributions of $10-20 \mathrm{DN}$ at $2.92 \mu \mathrm{m}$, and occasionally as high as $40 \mathrm{DN}$, but in most cases the residual signal in the core of the $\mathrm{B}$ ring is less than $5 \mathrm{DN}$ and comparable to the noise level in the data (see Section 6.4).

In a few cases, we find that the residual signal in the B ring is negative, a situation which is obviously difficult to explain with reflected sunlight or scattered Saturnshine. The most likely explanation here is a small decrease in the instrumental background, typically of 5-10 DN. This might be corrected by using a low-order polynomial fit to the measured instrumental background, as noted above. All instances where the residual signal level in the most opaque part of the B ring exceeds $2 \mathrm{DN}$ - either positive or negative - are identified by the code ' $\mathrm{D}$ ' in Table 8 .

\subsection{Stellar fluxes}

Although it is not necessary to know the absolute value of the stellar flux in order to calibrate and interpret occultation data, it is of some interest to compare the observed stellar fluxes with those expected based on the magnitudes of the occulted stars. We do this in two ways in Fig. 12. In the upper panel we plot the observed unocculted stellar signal $F_{0}$ (in DN per sample) at $2.92 \mu \mathrm{m}$ against that predicted based on the magnitude of the star at $K$-band (i.e., $2.2 \mu \mathrm{m}$ ) and the known integration time $\tau_{\mathrm{IR}}$. In the lower panel we 

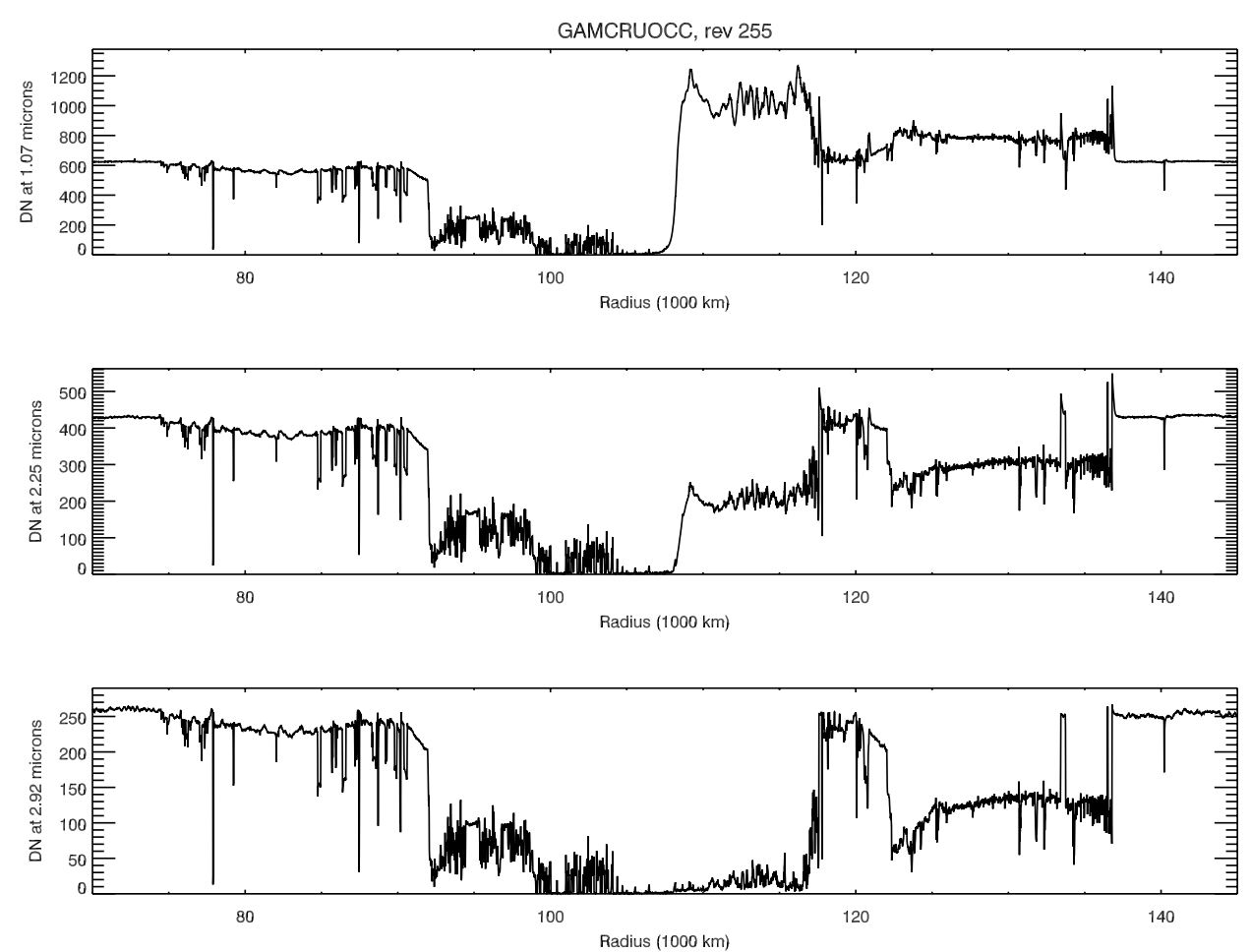

occ_plot_ov3chan.pro: Mon Apr 22 22:20:39 2019

Fig. 11.- Profiles for the ingress occultation of $\gamma$ Crucis on rev 255 at three wavelengths. The top panel shows the lightcurve at a wavelength of $1.07 \mu \mathrm{m}$, the middle panel is at $2.25 \mu \mathrm{m}$, while the bottom panel shows the lightcurve at our standard wavelength of $2.92 \mu \mathrm{m}$. At $1.07 \mu \mathrm{m}$ we see a combination of reflected light from the sunlit side of the rings plus starlight, whereas the flux at $2.92 \mu \mathrm{m}$ is almost entirely from the star. At $2.25 \mu \mathrm{m}$, the situation is intermediate, with the signal from the rings being less than it is at $1.07 \mu \mathrm{m}$. During the occultation of the $\mathrm{B}$ ring, at a radius of $\sim 108,000 \mathrm{~km}$, the star entered the planet's shadow on the rings. Interior to this radius, reflected light from the rings is absent and we see only the star at all three wavelengths. 
plot the observed unocculted stellar flux (in DN/sec/channel) at $2.92 \mu \mathrm{m}$ directly against the magnitude of the star at $K$-band. Because the absolute flux calibration for VIMS is uncertain for point sources such as stars, we have adopted a count rate for a 0-magnitude star so as to match the measured fluxes for the bright stars $\alpha$ Ori, $\alpha$ Sco, $\gamma$ Cru and o Ceti. In Table 2 we list the predicted unocculted count rates for the brightest stars observed multiple times by VIMS, along with their ranges of observed rates at $2.92 \mu \mathrm{m}$.

Fig. 12 shows that in most cases the observed count rates agree fairly well (i.e., within a factor of 2) with the predicted values. Points which fall well below the diagonal line generally reflect instances of poor spacecraft pointing, which reduces the flux falling in the targeted pixel (see below). However, several stars show count rates from multiple occultations which appear to fall systematically below the predicted values. The most serious cases are listed in Table 3, along with their spectral types. No obvious pattern emerges which might explain why these particular stars are fainter than predicted: the list includes both late-M giants (similar to our calibrators $\gamma$ Cru and o Ceti) as well as much hotter A0 and K1 stars. We note here that, while many of the late-type giants in the VIMS star catalog are known to be long-period variables - o Ceti being the type example - generally such stars vary much less in the near-infrared than they do at visible wavelengths.

In addition to those stars which appear systematically fainter than expected, eleven individual occultations yielded unocculted stellar count rates less than one-third of the predicted values: $\alpha$ Tau (28), R Leo (30 \& 68), $\gamma$ Cru (rev 77), $\alpha$ Tra (100), R Cas (185, $192 \& 243), \mathrm{R}$ Car (191) and $\lambda \mathrm{Vel}(245 \& 265)$. (These are the data points that fall below the $F_{\text {obs }}=\frac{1}{3} F_{\text {pred }}$ line in the lower right part of the figure's upper panel.) In most cases, the problem appears to have been poor spacecraft pointing and/or a problem with the onboard stellar acquisition.

Two extremely red objects, CW Leo and $\eta$ Car, have observed count rates that are badly underestimated by our model based on $K$-magnitudes. The seven data points well above the $F_{\text {obs }}=F_{\text {pred }}$ line in the leftmost part of Fig. 12 represent these two stars, whose $K$ magnitudes are +1.31 and +1.14 , respectively. These significantly underestimate the stars'

Table 2: Predicted and observed stellar count rates at $2.92 \mu \mathrm{m}$ for the brightest VIMS occultation stars, summed over 8 spectral channels and normalized to a common integration time $\left(\tau_{\mathrm{IR}}\right)$ of $40 \mathrm{~ms}$.

\begin{tabular}{|r|c|c||c|c|c|}
\hline Star & Spec. type & $K$ mag & $\tau_{\text {IR }}(\mathrm{ms})$ & DN (pred) & DN (obs) \\
\hline$\alpha$ Ori & M1 Ia & -4.00 & 40 & 1780 & $930-1940$ \\
$\alpha$ Sco & M1 Iab & -3.78 & 40 & 1460 & $840-1540$ \\
R Dor & M8 III & -3.41 & 40 & 1040 & $1050-1200$ \\
$\alpha$ Her & M5 II & -3.37 & 40 & 1000 & $720-750$ \\
W Hya & M8 e & -3.10 & 40 & 780 & $470-680$ \\
$\gamma$ Cru & M3 III & -3.04 & 40 & 740 & $200-720$ \\
o Cet & M7 e & -2.60 & 40 & 490 & $200-590$ \\
\hline
\end{tabular}



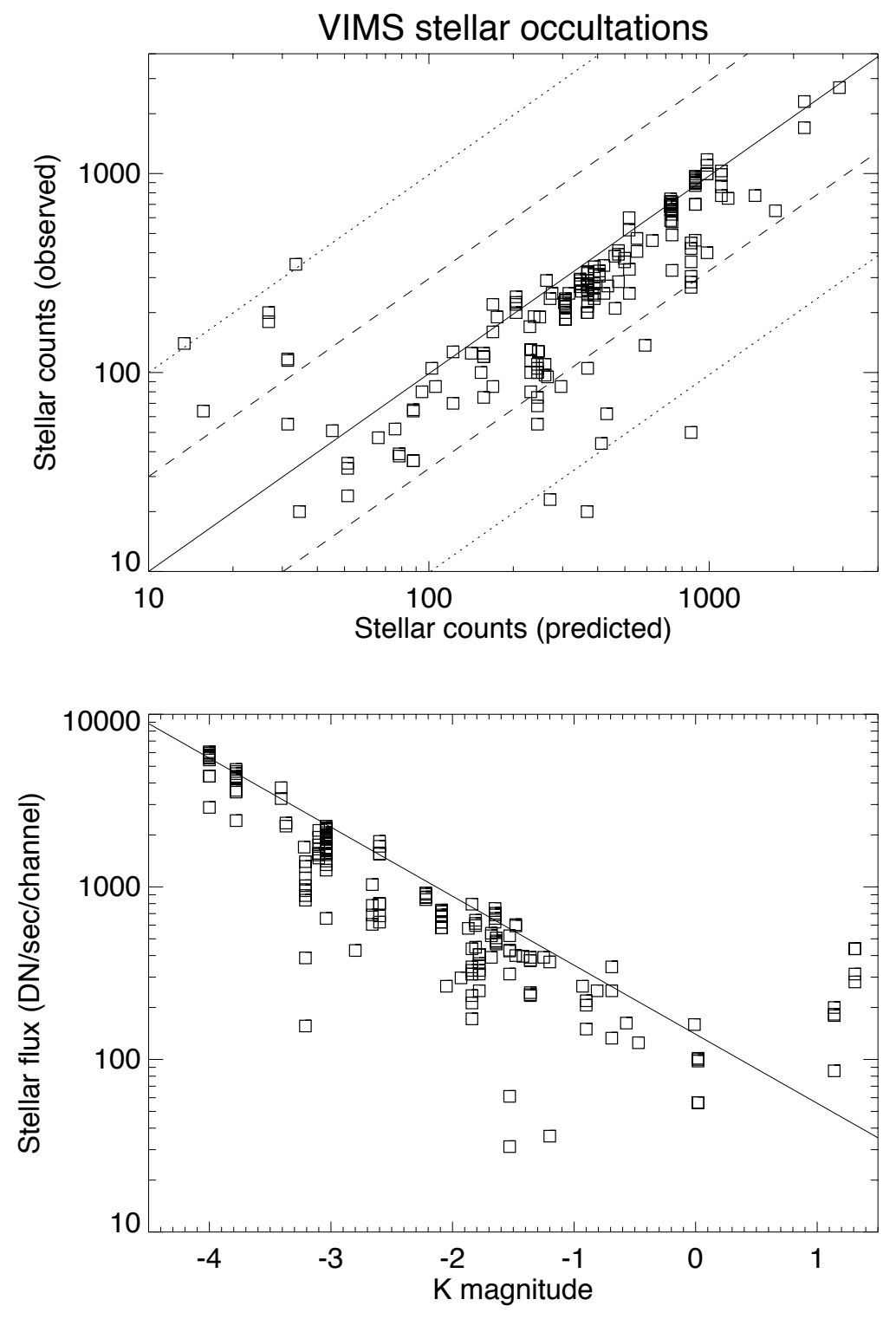

tablel_paper_rev.pro: Sat Apr 13 23:01:38 2019

Fig. 12.- Observed vs predicted stellar counts (in DN) for stars occulted by the rings. In the upper panel the observed counts for the unocculted star, summed over 8 spectral channels centered at a wavelength of $2.92 \mu \mathrm{m}$, are plotted vs the predicted counts based on the stellar magnitude and integration time. The solid diagonal line indicates equal count rates; most stars fall on or below this line, as expected. Dashed and dotted lines indicate counts that deviate by factors of 3 and 10 from the predicted values, respectively. In the lower panel, the observed stellar count rates (in DN/sec/channel) are plotted vs stellar $K$ magnitude. The straight line indicates our empirical calibration, fitted to the bright stars $\alpha$ Ori, $\alpha$ Sco, $\gamma$ Cru and o Ceti, which corresponds to $140 \mathrm{DN} / \mathrm{s}$ per spectral channel for a star of magnitude $K=0.0$. 
observed fluxes at $2.92 \mu \mathrm{m}$, and these stars are even brighter at wavelengths of $4-5 \mu \mathrm{m}$. CW Leo - more commonly known as IRC +10216 - is almost as bright at $4.25 \mu \mathrm{m}$ as is o Ceti at $2.92 \mu \mathrm{m}$.

An infrared spectral atlas containing many of the stars observed by VIMS during the course of Cassini's interplanetary cruise and saturnian orbital tour, reduced so far as is possible to absolute fluxes, has been published by Stewart et al. (2015).

\subsection{Photometric noise levels}

Except in cases of poor stellar-acquisition, where the noise level in the data is dominated by unpredictable, low-frequency pointing variations, the noise level in the reduced transmission profiles is dominated by a combination of detector read noise $N_{r}$ and shot noise in the stellar $\left(N_{*}\right)$ and instrumental background $\left(N_{b}\right)$ signals. The intrinsic read noise level of the VIMS-IR InSb detectors was measured to be $\sim 350$ electrons for short integration times, at focal-plane temperatures of $70 \mathrm{~K}$ or less. ${ }^{9}$ Some additional noise may arise from shot noise in the thermal emission from the (relatively warm) VIMS fore-optics, but both this and the spectrometer background are greatly reduced by the multi-segment blocking and order-sorting filter mounted above the detector array. Fortunately for our purpose, the filter segment immediately shortward of $3.1 \mu \mathrm{m}$ is particularly efficient, with no known blue or red leaks.

The gain level in the VIMS digital processing electronics was preset before launch so that the expected noise level for short integration times would correspond to approximately $1 \mathrm{DN}$. Allowing for the usual co-addition of 8 spectral channels, the expected read noise per occultation sample is then $N_{r} \simeq \sqrt{8} \simeq 2.83 \mathrm{DN}$. The shot noise in the stellar signal is given (in DN) by $N_{*}=\sqrt{g F} / g=\sqrt{F / g}$, where the gain factor $g \simeq 350$ converts the measured $\mathrm{DN}$ to detected electrons (or photons). A similar expression applies to the shot noise in

\footnotetext{
${ }^{9}$ Based on data provided by the manufacturer from tests of the focal plane assembly. The actual operating temperature of the focal plane was usually around $60 \mathrm{~K}$, and very stable. At integration times of $0.1 \mathrm{~s}$ and longer, irrelevant to occultation experiments, the noise level increases roughly as $1 / f$.
}

Table 3: Predicted and observed stellar count rates at $2.92 \mu \mathrm{m}$ for stars that are systematically fainter than expected, summed over 8 spectral channels and normalized to a common integration time of $40 \mathrm{~ms}$.

\begin{tabular}{|r|c|r||c|c|c|}
\hline Star & Spec. type & $K$ mag & $\tau_{\text {IR }}(\mathrm{ms})$ & DN (pred) & DN (obs) \\
\hline R Leo & M8 III & -3.21 & 40 & 860 & $50-460$ \\
R Cas & M7 III & -1.84 & 40 & 245 & $55-250$ \\
L $^{2}$ Pup & M5 III & -1.78 & 40 & 230 & $80-130$ \\
X Oph & K1 III & -0.90 & 40 & 102 & $50-70$ \\
$\alpha$ Lyr & A0 V & 0.02 & 40 & 44 & $17-30$ \\
\hline
\end{tabular}


the instrumental background signal in each measurement, $N_{b}$, but not to the subtracted background because here we use an average value, as described above. The exact source of the instrumental background signal is uncertain, as it is not simply proportional to the integration time as would be expected for dark current or thermal emission from the spectrometer optics. It appears instead to be primarily an electronic offset introduced by the multiplexer or data-processing hardware in order to avoid negative signals going into the analog-to-digital converter. Based on empirical data, we can model this background as $B=B_{0}+B_{1} \times \tau_{\mathrm{IR}}$, where $B_{0}=900 \mathrm{DN}, B_{1}=1.0 \mathrm{DN} / \mathrm{ms}$ at $2.92 \mu \mathrm{m}$ and $\tau_{\mathrm{IR}}$ is the integration time. We assume that there is shot noise only in the second term, given by $N_{b}=\sqrt{g\left(B-B_{0}\right)} / g=\sqrt{\left(B-B_{0}\right) / g}$. The expected overall noise level in the stellar flux is therefore $N=\sqrt{N_{r}^{2}+N_{*}^{2}+N_{b}^{2}}$ (in DN), and the corresponding signal-to-noise level in the unocculted flux is $\sim F_{0} / N$.

For a definite example, let us consider an occultation by a typical bright star, $\gamma$ Crucis, for which we find that $F_{0} \simeq 2300 \mathrm{DN} / \mathrm{s} /$ channel at $2.92 \mu \mathrm{m}$, or $720 \mathrm{DN}$ in a typical $40 \mathrm{~ms}$ integration time and summed over 8 spectral channels (cf. Fig. 4). A typical instrumental background level is $940 \mathrm{DN}$ per sample of summed data, of which $900 \mathrm{DN}$ is the electronic offset. These translate into expected shot noise levels of $N_{*}=\sqrt{720 / 350} \simeq 1.43 \mathrm{DN}$, $N_{b}=\sqrt{40 / 350} \simeq 0.34 \mathrm{DN}$ and an overall noise level of $N \simeq 3.18 \mathrm{DN}$ for the unocculted star. The unocculted SNR is therefore $\sim 225$ per sample. The corresponding value when the star is fully occulted (see discussion below) is $N=\sqrt{N_{r}^{2}+N_{b}^{2}} \simeq 2.85 \mathrm{DN}$, or $0.40 \%$ of the unocculted stellar flux. For all but the brightest stars, both the unocculted and fully-occulted noise levels are effectively determined by read noise, and in fact only $N_{r}$ is taken into account in the standard occultation data sets delivered to the PDS.

Another source of unexpected noise in a few of the VIMS occultation lightcurves is an excessive number of charged particle impacts on the InSb detectors. In practice, this is most noticeable for ranges less than $\sim 240,000 \mathrm{~km}$, or $4 R_{S}$, and when the spacecraft is on a near-equatorial orbit. Examples include the ingress portions of the occultations of $\alpha$ Sco (13), 30 Psc (222) and o Cet (231), as well as $\delta$ Vir (29) and $\alpha$ Ori (46). This particular source of noise is readily detectable, as it is always positive and is uncorrelated between different spectral channels. Although we have not attempted to do so, it might be possible to exploit these two facts to identify and remove such particle hits with an automated routine. Instances of unusual levels of high-frequency noise are identified by the quality code ' $\mathrm{N}$ ' in Table 8.

\subsection{Minimum detectable optical depth}

The photometric noise level in the unocculted stellar signal determines the minimum normal optical depth which is, in principle, detectable in a particular occultation experiment. In a single sample, the $3-\sigma$ detection limit is given by the requirement that the decrease in stellar flux due to the rings equal or exceed three times the noise level in the 
data, or

$$
F_{0}\left(1-e^{-\tau / \mu}\right) \geq 3 N
$$

where $\tau$ is the normal optical depth and $\mu=\sin \left|B_{*}\right|$. As long as $\tau / \mu \ll 1$ we then have

$$
\tau_{\min } \simeq 3\left(N / F_{0}\right) \sin \left|B_{*}\right| .
$$

If the measured transmission profile is averaged over $n$ samples to, for example, a resolution of $1 \mathrm{~km}$, then $N / F_{0}$ and thus $\tau_{\text {min }}$ are reduced by a factor of $\sqrt{n} .{ }^{10}$ Note that the occultations that are most sensitive to low- $\tau$ material are those with low inclination angles $B_{*}$. Physically, this is because such occultations provide a longer slant path for the photons as they traverse the rings, and thus a greater opportunity for absorption by very low optical depth regions.

However, such purely statistical estimates of $\tau_{\min }$ refer primarily to localized structures (such as narrow ringlets); it is much more difficult to distinguish a very broad, low-optical depth feature from variations in the stellar signal due to low-frequency spacecraft pointing variations. Care should always be taken when such features are interpreted as real structures in the rings to obtain independent confirmation from other occultations.

As an example, consider again a $\gamma$ Cru occultation such as that in Fig. 4, where we have $F_{0}=720 \mathrm{DN}$ in each $40 \mathrm{msec}$ integration and $B_{*}=-62.35^{\circ}$. In this case the expected noise level for the unocculted star is $3.18 \mathrm{DN}$ per sample (see Section 6.4 above). With a mean radial velocity $v_{\text {rad }}=6.7 \mathrm{~km} / \mathrm{s}$, there are 3.7 samples per $\mathrm{km}$, from which we estimate a minimum 3- $\sigma$ detectable optical depth of 0.0061 at $1 \mathrm{~km}$ radial resolution or 0.0019 at $10 \mathrm{~km}$ resolution. This estimate is supported by experience, at least for the highest-quality observations. Actual data from the $\gamma$ Cru occultation on rev 89 show the apparent optical depth outside the A ring (between 137,000 and 144,000 km, excluding the F ring) at $10 \mathrm{~km}$ resolution to have an average value of 0.0025 and a peak-to-peak variation of \pm 0.0035 . These values compare favorably with the predicted minimum detectable optical depth estimated above.

In Table 4 we list representative values of $\tau_{\min }$ for the seven stars observed by VIMS that are most sensitive to low-optical depth material. These are based on the observed stellar count rates listed in Table 8 and plotted in Fig. 12 and correspond to 3- $\sigma$ detection limits at radial resolutions of $1 \mathrm{~km}$ and $10 \mathrm{~km}$. ( $\gamma$ Cru is also included here for reference purposes.) As noted above, the smallest values of $\tau_{\min }$ are obtained for occultations by low-inclination stars such as $o$ Ceti (Mira), $\alpha$ Ori (Betelgeuse), $\alpha$ Her, R Leo and 30 Psc, all of which have $\left|B_{*}\right|<15^{\circ}$. However, the large count rates for $\alpha$ Sco and W Hya, along with low radial velocities for some of their occultations, ${ }^{11}$ make them comparably sensitive despite their larger values of $\left|B_{*}\right|$. For some occultations by $o$ Ceti and $\alpha$ Ori, $\tau_{\min } \leq 0.0010$ at $1 \mathrm{~km}$ resolution and $\leq 0.0003$ at $10 \mathrm{~km}$. Such events are especially valuable in probing

\footnotetext{
${ }^{10}$ The total stellar counts $F_{0}$ are increased by a factor of $n$ while the noise increases by $\sqrt{n}$, leading to an overall improvement in SNR of $\sqrt{n}$.

${ }^{11}$ The smaller the projected radial velocity of the star behind the rings, the larger the number of samples that are averaged per $\mathrm{km}$.
} 
low-optical depth regions such as the $\mathrm{D}$ and $\mathrm{F}$ rings as well as the numerous gaps in the $\mathrm{C}$ ring and the Cassini Division.

The greatest sensitivity to low- $\tau$ material for any of the VIMS stellar occultations is provided by a series of four distant, slow chord occultations by $o$ Cet on revs 8-12, with $B_{*}=3.45^{\circ}$. Fig. 13 shows the ingress and egress profiles from the $o$ Cet occultation on rev 8 for the inner part of the Cassini Division, at $1 \mathrm{~km}$ resolution. The upper panel shows, from left to right, the $300 \mathrm{~km}$-wide Huygens gap and its two embedded ringlets, the Herschel gap and its ringlet, and the narrower Russell, Jeffreys and Kuiper gaps (Colwell et al. 2009). The lower panel shows the Huygens gap and ringlet at an expanded vertical scale. The noise level here, as well as that seen exterior to the F ring (not shown here), is consistent with the predicted value of $\tau_{\min }=0.0004$ for this occultation, except for the slightly negative optical depths near the outer edge of the gap. These are due to diffraction by the adjacent ring material (Becker et al. 2016; Harbison et al. 2019). Unfortunately, none of this series of $o$ Cet occultations provided any data on the $\mathrm{C}$ or $\mathrm{D}$ rings.

The most sensitive VIMS occultations to material in the $\mathrm{C}$ ring are those of $\alpha$ Orionis, for which $B_{*}=11.68^{\circ}$. For the best of these occultations $\tau_{\min }=0.0007$ at $1 \mathrm{~km}$ resolution and $\sim 0.0002$ at $10 \mathrm{~km}$ resolution, ten times lower than for our numerical example of $\gamma$ Cru. We illustrate this sensitivity in Fig. 14, whose upper panel shows an optical depth profile of the innermost part of the $\mathrm{C}$ ring from an ingress occultation of $\alpha$ Ori on rev 277. This figure shows four narrow, unnamed gaps embedded within a region whose average optical depth is only $\sim 0.030$. Interior to the inner edge of the $\mathrm{C}$ ring at $74,490 \mathrm{~km}$ the noise level is consistent with the predicted value of $\tau_{\min }=0.0009$, as shown in the lower panel of the figure. Note that all of the narrow gaps in this region show distinctly negative optical depths; again most likely due to diffraction by small particles in the adjacent parts of the ring.

As a final example of the minimum detectable optical depth achievable in the best VIMS data sets, Fig. 15 shows a profile of the narrow F ring, from the same occultation by $\alpha$ Ori on rev 277. Here the F ring's tenuous interior and exterior shoulders, also referred to as 'strands', are readily visible at the expanded scale shown in the lower panel despite their optical depths of 0.005 or less.

\subsection{Maximum detectable optical depth}

It is more difficult to quantify the maximum normal optical depth which is meaningfully detectable. This marks the threshold at which any stellar signal is detected, and depends on both the residual photometric noise level $N$ and on how precisely any non-stellar background can be determined for the particular occultation experiment. If we assume, for the present, that the background level is known perfectly - including any contribution from the rings - then in a single sample, the $3-\sigma$ detection limit is given by the requirement that the residual stellar flux equal or exceed three times the noise level in the data, or

$$
F_{0} e^{-\tau / \mu} \geq 3 N,
$$



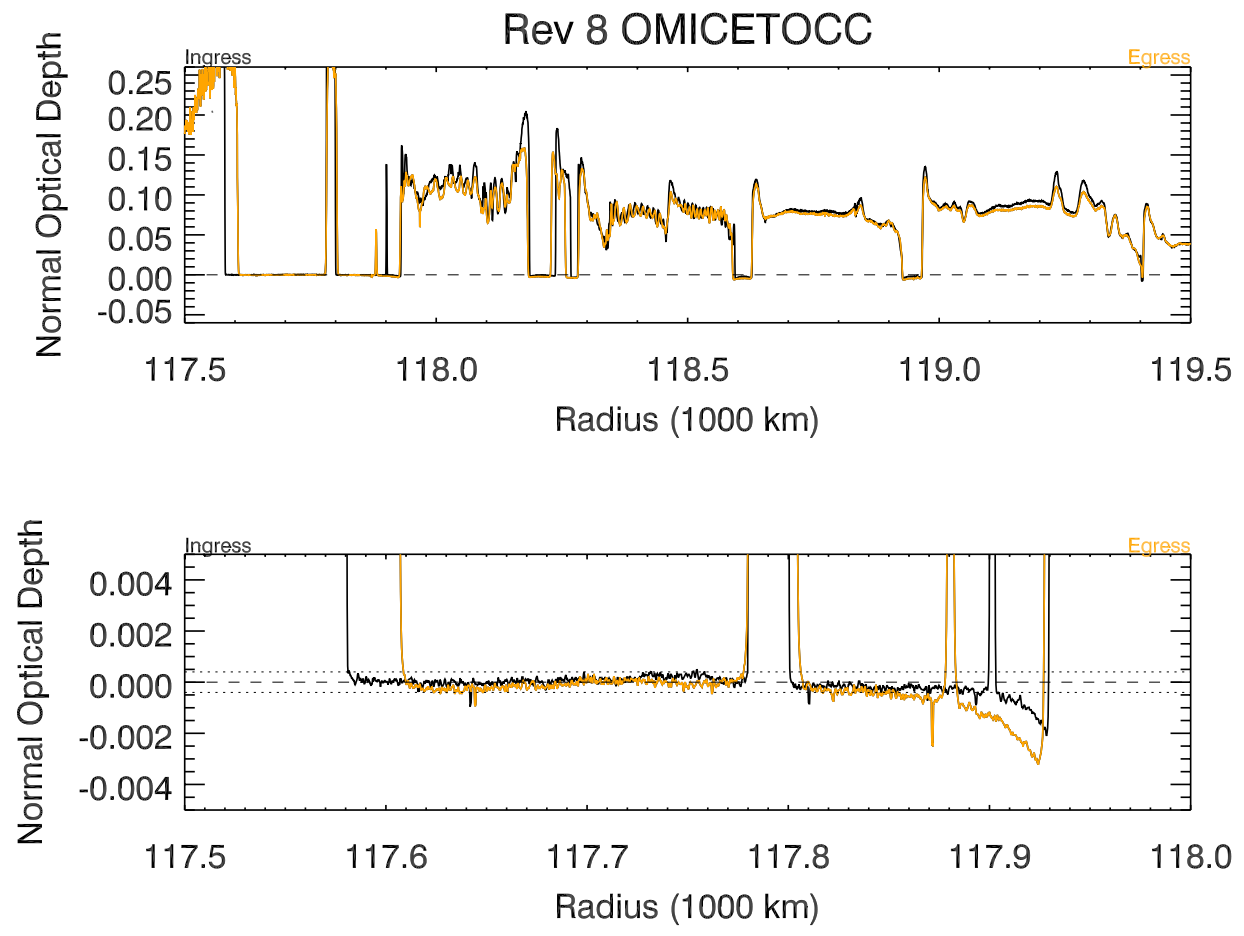

Fig. 13. - Ingress and egress optical depth profiles of the inner Cassini Division at $1 \mathrm{~km}$ resolution are shown in the upper panel, as obtained on rev 8 with the bright, low-inclination star $o$ Ceti. The data are summed over 8 spectral channels, with a central wavelength of $2.92 \mu \mathrm{m}$. The complete light curves are shown in Fig. 6. Ingress and egress data are overplotted here for easier comparison, ingress in black and egress in orange/grey. The lower panel shows the $300 \mathrm{~km}$-wide Huygens gap at an expanded vertical scale, with the predicted minimum detectable optical depth in these data of $\tau_{\min }=0.0004$ indicated by the dotted lines. For this occultation, $\tau_{\max }=0.31$, which is exceeded here only by the Huygens ringlet. 

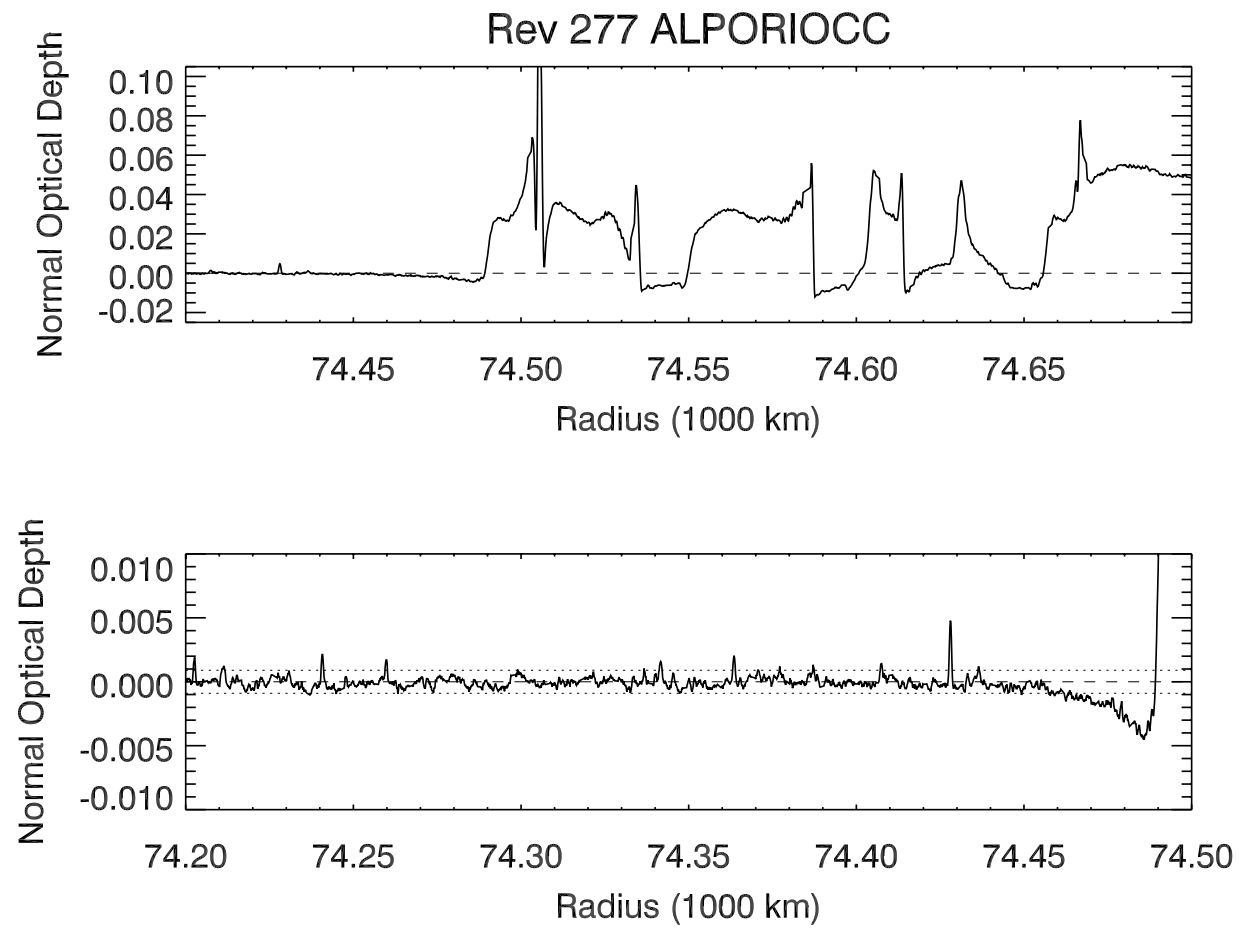

Fig. 14.- An optical depth profile of the innermost part of the $\mathrm{C}$ ring at $1 \mathrm{~km}$ resolution, obtained from an occultation of the bright star $\alpha$ Ori on rev 277. The data are summed over 8 spectral channels, centered at a wavelength of $2.92 \mu \mathrm{m}$. The integration time was $20 \mathrm{~ms}$, and the range to Cassini was $755,000 \mathrm{~km}$. The unocculted signal level is $970 \mathrm{DN}$. The lower panel shows the $300 \mathrm{~km}$-wide region immediately interior to the $\mathrm{C}$ ring's inner edge at an expanded vertical scale, with the predicted minimum detectable optical depth of $\tau_{\min }=0.0009$ indicated by the dotted lines. For this occultation, $\tau_{\max }=1.12$ and the region between 73,500 and $74,300 \mathrm{~km}$ was used to normalize the stellar transmission profile, rather than the usual region exterior to the $\mathrm{F}$ ring. 

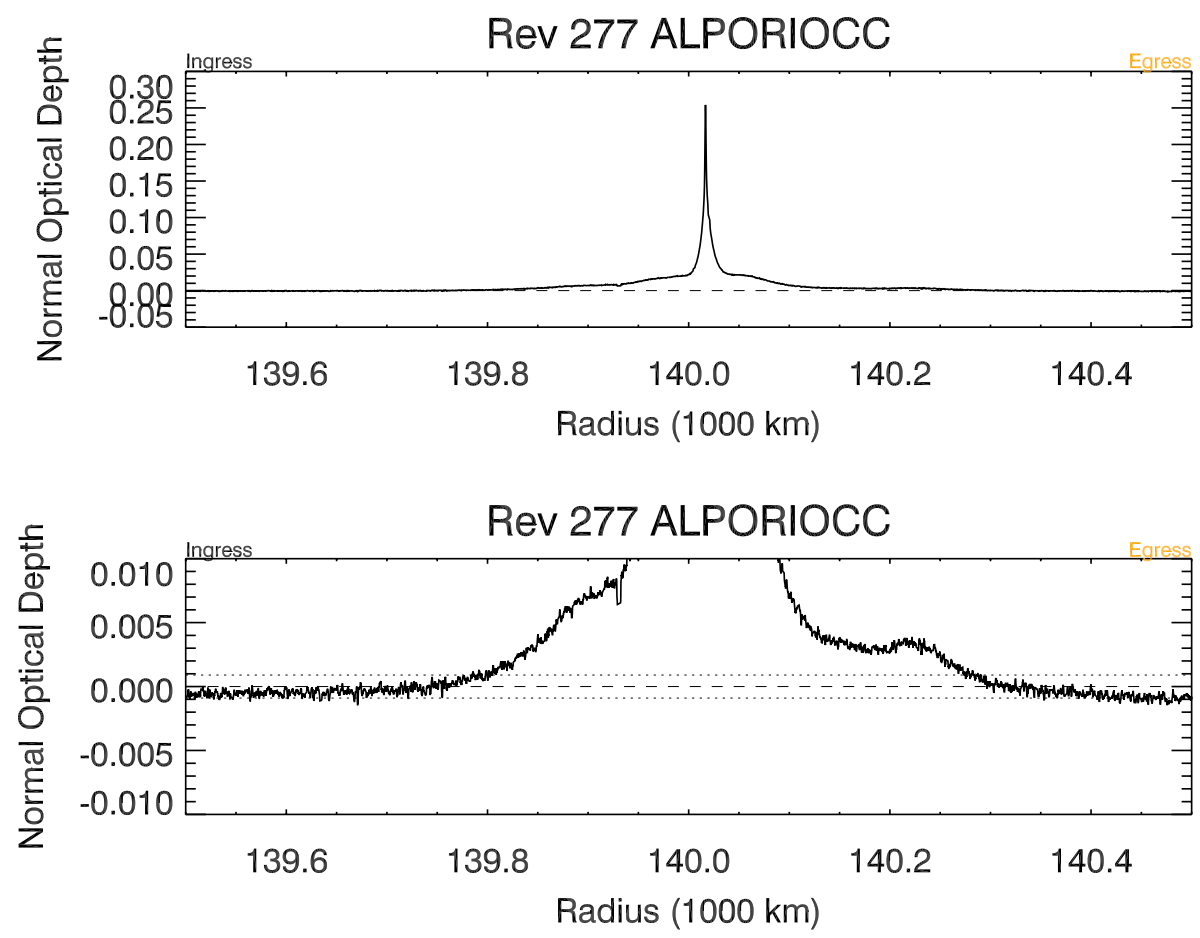

Fig. 15.- An optical depth profile of the narrow $\mathrm{F}$ ring at $1 \mathrm{~km}$ resolution, obtained from the same occultation of $\alpha$ Ori on rev 277 used in Fig. 14. Again, the data are summed over 8 spectral channels, centered at a wavelength of $2.92 \mu \mathrm{m}$. The lower panel shows the same region at an expanded vertical scale, with the predicted minimum detectable optical depth of $\tau_{\min }=0.0009$ indicated by the dotted lines. For this occultation, $\tau_{\max }=1.12$ and the region between 142,00 and 143,000 $\mathrm{km}$ was used to normalize the stellar transmission profile. 
where again $\tau$ is the normal optical depth and $\mu=\sin \left|B_{*}\right|$. We can then write the formal $3-\sigma$ detection limit as

$$
\tau_{\max }=\ln \left(F_{0} / 3 N\right) \sin \left|B_{*}\right|,
$$

where here the noise level $N$ is evaluated for zero stellar flux. If the measured transmission profile is averaged over $n$ samples then the quantity $F_{0} / N$ is increased by a factor of $\sqrt{n}$. In this case, the occultations most likely to penetrate the more opaque ring regions are those with the largest values of $\left|B_{*}\right|$, because the slant path and thus the loss of stellar signal is minimized.

As an example, consider again an occultation by $\gamma$ Cru such as that in Fig. 4, where we have $F_{0}=720 \mathrm{DN}$ in a $40 \mathrm{msec}$ integration and $B_{*}=-62.35^{\circ}$. In this case the expected noise level for the fully-occulted star is $2.85 \mathrm{DN}$ per sample (see Section 6.4 above). With a mean radial velocity of $v_{\mathrm{rad}}=6.7 \mathrm{~km} / \mathrm{s}$, there are 3.7 samples per $\mathrm{km}$, from which we estimate a $3-\sigma$ threshold optical depth of 4.49 at $1 \mathrm{~km}$ resolution or 5.51 at $10 \mathrm{~km}$ resolution. As may be seen in Fig. 4 above, the normal optical depth clearly reaches or exceeds the latter value over much of the central B ring.

In Table 5 we list representative values of $\tau_{\max }$ for the four stars observed by VIMS that are most able to probe high-optical depth regions such as the B ring. As in Table 4, these are based on the observed stellar count rates listed in Table 8 and plotted in Fig. 12 and correspond to $3-\sigma$ detection limits at radial resolutions of $1 \mathrm{~km}$ and $10 \mathrm{~km} .^{12}$ The most sensitive probes of high optical depth regions are provided by bright, high-inclination stars such as $\gamma$ Cru, R Dor, $\alpha$ Cen, $\epsilon$ Mus and $\mu$ Cep, all of which have $\left|B_{*}\right|>55^{\circ}$. In practice, however, there were no occultations of $\mathrm{R}$ Dor that crossed the central $\mathrm{B}$ ring, so that by far the best VIMS occultation data for this region are provided by the 17 dark-side (i.e., pre-2009) ingress occultations of $\gamma$ Crucis, many of which have $\tau_{\max } \simeq 4.5$ at a resolution of $1 \mathrm{~km}$, and $\sim 5.5$ at $10 \mathrm{~km}$.

Unlike the situation for $\tau_{\min }$, which scales as $\sin \left|B_{*}\right| / F_{0}$ and where a larger stellar count rate can compensate for a higher value of $\left|B_{*}\right|, \tau_{\max }$ depends directly on $\sin \left|B_{*}\right|$ but only logarithmically on the unocculted stellar flux $F_{0}$. In consequence, even very bright stars at low inclinations have relatively low values of $\tau_{\max }$. For reference purposes, and also to illustrate the strong dependence of $\tau_{\max }$ on $B_{*}$, we include in Table 5 data for the very bright, low-inclination stars $\alpha$ Sco, $\alpha$ Ori and o Ceti. Only for $\alpha$ Sco does $\tau_{\max }$ ever exceed 3.0, and for $\alpha$ Ori (our brightest star) it is never greater than 1.4.

In Fig. 16, we show four high-quality optical depth profiles of a part of the B2 region in the central $B$ ring, binned to a uniform radial resolution of $1 \mathrm{~km}$. In this region, the normal optical depth alternates between a value around 2.0 and a much higher level which is effectively opaque. Three of these profiles are from dark-side $\gamma$ Cru occultations on revs 79,86 and 94, with negligible ring background, and the fourth is from a sunlit occultation

\footnotetext{
${ }^{12}$ For a given radial resolution, both $\tau_{\min }$ and $\tau_{\max }$ also depend weakly on the integration time, $\tau_{\mathrm{IR}}$, even though the total stellar and background signals are unchanged, due to the reduction in read noise as the number of co-added samples is reduced.
} 
Table 4: Minimum detectable optical depths in selected VIMS ring occultations at resolutions of $1 \mathrm{~km}$ and $10 \mathrm{~km}$.

\begin{tabular}{|c|c|c|c|c|c|c|}
\hline Obs. ID & $K$ mag & $\overline{B_{*}}$ & $\begin{array}{c}v_{\mathrm{rad}} \\
(\mathrm{km} / \mathrm{s})\end{array}$ & $\begin{array}{c}\tau_{\mathrm{IR}} \\
(\mathrm{ms})\end{array}$ & $\begin{array}{l}\tau_{\min }^{a} \\
(1 \mathrm{~km})\end{array}$ & $\begin{array}{l}\tau_{\min }^{a} \\
(10 \mathrm{~km})\end{array}$ \\
\hline o Cet (8) & -2.60 & 3.45 & 5.7 & 80 & 0.0004 & 0.00013 \\
\hline$\alpha$ Ori $(245)$ & -4.00 & 11.68 & 5.0 & 20 & 0.0007 & 0.00022 \\
\hline$\alpha \operatorname{Sco}(237)$ & -3.78 & -32.16 & 2.4 & 60 & 0.0010 & 0.00033 \\
\hline$\alpha \operatorname{Her}(211)$ & -3.37 & 9.27 & 7.0 & 20 & 0.0015 & 0.0005 \\
\hline R Leo (63) & -3.21 & 9.55 & 8.7 & 40 & 0.0025 & 0.0008 \\
\hline W Hya (236) & -3.10 & -34.64 & 1.8 & 60 & 0.0025 & 0.0008 \\
\hline $30 \operatorname{Psc}(222)$ & -0.47 & -1.06 & $(7.0)$ & 20 & 0.0030 & 0.0009 \\
\hline$\gamma \mathrm{Cru}(89)$ & -3.04 & -62.35 & 6.7 & 40 & 0.0061 & 0.0019 \\
\hline
\end{tabular}

Table 5: Maximum detectable optical depths in selected VIMS ring occultations at resolutions of $1 \mathrm{~km}$ and $10 \mathrm{~km}$.

\begin{tabular}{|r|r|r|c||c|l|l|}
\hline Obs. ID & $K$ mag & $B_{*}$ & $\begin{array}{c}v_{\text {rad }} \\
(\mathrm{km} / \mathrm{s})\end{array}$ & $\begin{array}{c}\tau_{\mathrm{IR}} \\
(\mathrm{ms})\end{array}$ & $\begin{array}{l}\tau_{\max }^{a} \\
(1 \mathrm{~km})\end{array}$ & $\begin{array}{l}\tau_{\max }^{a} \\
(10 \mathrm{~km})\end{array}$ \\
\hline$\gamma$ Cru (82) & -3.04 & -62.35 & 6.6 & 40 & 4.51 & 5.53 \\
$\alpha$ Cen (66) & -1.48 & -67.30 & 4.9 & 60 & 3.81 & 4.87 \\
$\epsilon$ Mus (94) & -1.42 & -72.77 & 3.6 & 60 & 3.69 & 4.79 \\
$\mu$ Cep (193) & -1.65 & 59.90 & 4.8 & 40 & 3.60 & 4.59 \\
\hline$\alpha$ Sco (243) & -3.78 & -32.16 & 9.3 & 20 & 2.79 & 3.40 \\
$\alpha$ Ori (277) & -4.00 & 11.68 & 10.1 & 20 & 1.12 & 1.35 \\
o Cet (231) & -2.60 & 3.45 & 9.4 & 20 & 0.21 & 0.28 \\
\hline
\end{tabular}

${ }^{a} 3-\sigma$ limits (see text). 
of $\alpha$ Sco on rev 239. They were chosen to illustrate the effects of integration time and ring opening angle on $\tau_{\max }$. For the profiles illustrated here, $\tau_{\max }$ varies from 2.76 for the intermediate-inclination $\alpha$ Sco occultation to between 3.98 and 4.65 for the $\gamma$ Cru occultations, which vary in integration time from 20 to $60 \mathrm{~ms}$ per sample. As expected, the parts of the profiles below the 3- $\sigma$ threshold level show very similar structure, while the parts at and above the 1- $\sigma$ level are mostly noise. The apparent optical depths in these opaque regions are determined primarily by $\tau_{\max }$, and do not at all reflect the true opacity of the ring. The feature at 101,480-101,550 km is especially revealing, as its peak optical depth straddles the $\tau_{\max }$ level for the upper two profiles but is clearly truncated in the lower profiles.

In the core of the B ring (the region referred to as B3 by Colwell et al. (2009), located between radii of 104,000 and $110,000 \mathrm{~km}$ ) the residual signal is $<1 \mathrm{DN}$ in several of the highest-quality $\gamma$ Cru occultations and the calculated optical depth is above $\tau_{\max }$ almost everywhere in this region, except for occasional 50-km-wide 'windows' where it drops to between 2 and 3. It seems likely that the true optical depth may well exceed 6 over a significant fraction of B3. ${ }^{13}$ This is consistent with estimates by the Cassini UVIS team of $\tau \geq 4.6$ in the central B ring based on early stellar occultation observations (Colwell et al. 2007), and with a 1- $\sigma$ estimate of $\tau_{\max } \sim 8$ from an occultation by the very bright, high-latitude star $\beta$ Cen (see Fig. 10 in Colwell et al. (2010)).

We note in passing that these estimates are much higher than the lower limit set for the central B ring by the single Voyager stellar occultation of $\tau \geq 2.5$ (Esposito et al. 1983a,b). The signal-to-noise ratio of the Voyager occultation, however, was much lower than that of the best Cassini data sets, with a maximum count rate of $39 \mathrm{DN}$ per $10 \mathrm{~ms}$ sample. Similar, rather low values for $\tau$ in the central B ring were inferred from the Earth-based occultation of $28 \mathrm{Sgr}$ in 1989 (Harrington et al. 1993; Nicholson et al. 2000), but these data may have been unavoidably contaminated by residual levels of scattered light from the rings, since telluric absorption by water vapor makes it impossible to make Earth-based observations in the strongest water ice bands.

It is important to keep in mind, however, that the maximum apparent value of the optical depth in any particular occultation can be strongly affected by even a small unmodeled background (i.e., non-stellar) contribution to the measured flux. For example, an unrecognized background signal of $10 \mathrm{DN}$ in our canonical example of a $\gamma$ Cru occultation would lead to an apparent maximum optical depth of $\tau^{\prime}=\mu \ln (720 / 10) \simeq 3.78$, where $\mu=\sin \left|B_{*}\right|=0.886$. Unlike $\tau_{\max }$, this apparent optical depth is not affected by binning the data at lower resolution, at least in the presence of a constant background signal. A concrete example is shown in Fig. 17, where the predicted value of $\tau_{\max }=3.09$ at $10 \mathrm{~km}$ resolution, while the apparent value of $\tau$ does not exceed 2.0, even in the B3 region. Careful inspection of the lightcurve shows that there is a nearly-constant residual signal of $\sim+10 \mathrm{DN}$ across the B2 and B3 regions (i.e., 99,000 - 110,000 km), likely due either to reflected light from

\footnotetext{
${ }^{13}$ At $\tau=6.0$, the residual stellar signal $F_{0} e^{-\tau / \mu} \simeq 0.82 \mathrm{DN}$, while at $10 \mathrm{~km}$ resolution the averaged read noise $N_{r}=\sqrt{8 / 37} \simeq 0.46 \mathrm{DN}$.
} 

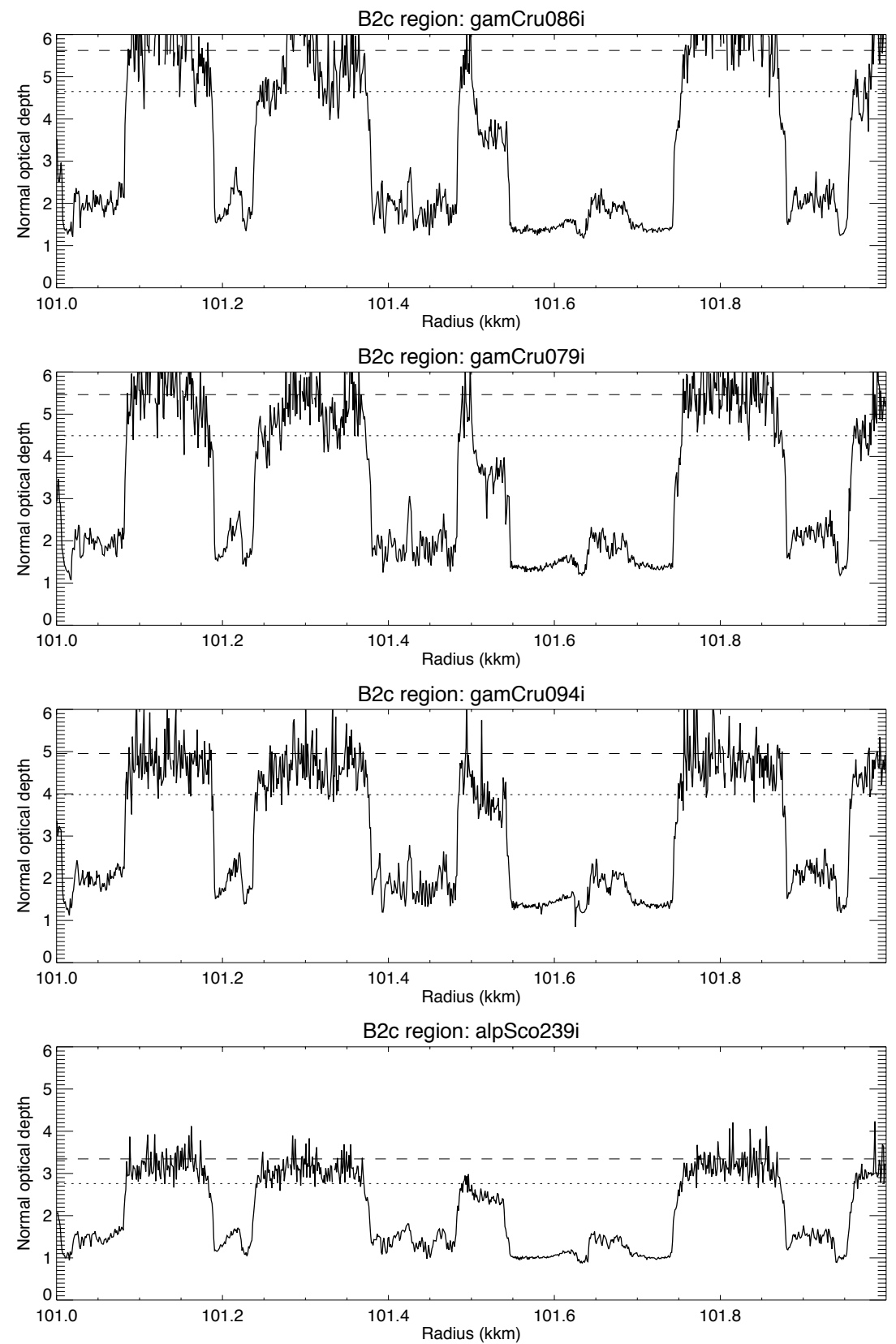

plot_Bring.pro: Mon Apr 29 17:20:21 2019

Fig. 16. - Optical depth profiles of a $1000 \mathrm{~km}$ segment of the B2 region in the central B ring obtained from the $\gamma$ Cru occultations on revs 79,86 and $94\left(B_{*}=-62.35^{\circ}\right)$, plus an $\alpha$ Sco occultation on rev $239\left(B_{*}=-32.16^{\circ}\right)$, all binned to a radial resolution of $1 \mathrm{~km}$. Again, the data shown are summed over 8 channels centered at a wavelength of $2.92 \mu \mathrm{m}$. Dashed and dotted lines indicate the 1- $\sigma$ and 3- $\sigma$ values of $\tau_{\max }$ for each observation. The $\gamma$ Cru profiles are arranged in order of decreasing integration time, from $60 \mathrm{~ms}$ to $20 \mathrm{~ms}$, with corresponding values of $\tau_{\max }=4.65,4.49$ and 3.98, respectively, while $\tau_{\max }=2.75$ for the $\alpha$ Sco occultation. 
the rings or to an unrecognized variation in the instrumental background signal. By being attentive to such suspicious circumstances where the transmission seems to "bottom out" at a constant but non-zero value - or where the optical depth appears to reach a maximum which is well short of $\tau_{\max }$ - one can usually identify occultations where there is a significant non-stellar background signal. Such cases are labeled with the quality code ' $D$ ' in Table 8 , described below, and flagged by a Note in the descriptive text files delivered to the PDS.

Situations in which the residual signal in the B ring is negative (such as might be caused by an overestimated instrumental background) are also fairly easy to spot, but result in a systematic overestimate of the optical depth. They are much less common than positive residuals, but can be recognized by the apparent stellar transmission $T$ going below zero in the core of the $\mathrm{B}$ ring. These observations are also labeled with the quality code ' $\mathrm{D}$ ' in Table 8.

Since $\tau_{\min }$ is smallest for low-inclination (i.e., small $\left|B_{*}\right|$ ) stars while, other things being equal, $\tau_{\max }$ is largest for stars with high inclinations (i.e., large $\left|B_{*}\right|$ ), there is no "ideal" occultation star. Rather, a mix of stellar inclinations is desirable and this was one goal of the VIMS stellar occultation program. For VIMS, $\alpha$ Sco (Antares) was perhaps the best overall compromise, with $K=-3.78$ and an intermediate value of $B_{*}=-32.16^{\circ}$.

\section{Data tables}

A complete list of all 190 ring stellar occultations observed (or attempted) with Cassini VIMS is provided in the Appendix as Table 8, including the star name and Cassini orbit number (or 'rev'), the start time and duration of the observation, the integration time used, whether or not the data were spectrally-summed or edited and the measured signal level for the unocculted star $F_{0}$. Additional notes provide a shorthand description of the radial coverage of the occultation. An alpha-numeric code indicates the quality of the data, based on a subjective visual evaluation, with a ' 1 ' signifying data of excellent quality, ' 2 ' indicating an average-quality light curve, and ' 3 ' data of poor quality that should not be used for photometric measurements (though they may be satisfactory for determining the locations of sharp features such as gap edges). An appended ' $\mathrm{V}$ ' indicates that the stellar baseline varies by more than $\sim 2 \%$, a ' $\mathrm{B}$ ' indicates that the instrumental background either is noisy or shows significant variations during the occultation, a ' $\mathrm{D}$ ' denotes a residual (presumably non-stellar) signal at $2.92 \mu \mathrm{m}$ of $2 \mathrm{DN}$ or more in magnitude in the most opaque parts of the B ring, and an ' $\mathrm{N}$ ' that the data are unusually noisy (generally because of a high rate of charged-particle hits). A ' - ' in this column indicates that no useful data were obtained.

A total of 21 occultations are classified as being of 'poor' quality, or $\sim 10 \%$ of the complete data set. In many, but not all, of these cases the culprit appears to be a bad stellar acquisition, as indicated by a lower-than-expected stellar flux (see discussion in Section 6.3 above). In other cases it is possible that the spacecraft pointing was less stable than usual 
VIMS_2013_046_WHYA_I_TAU_10KM
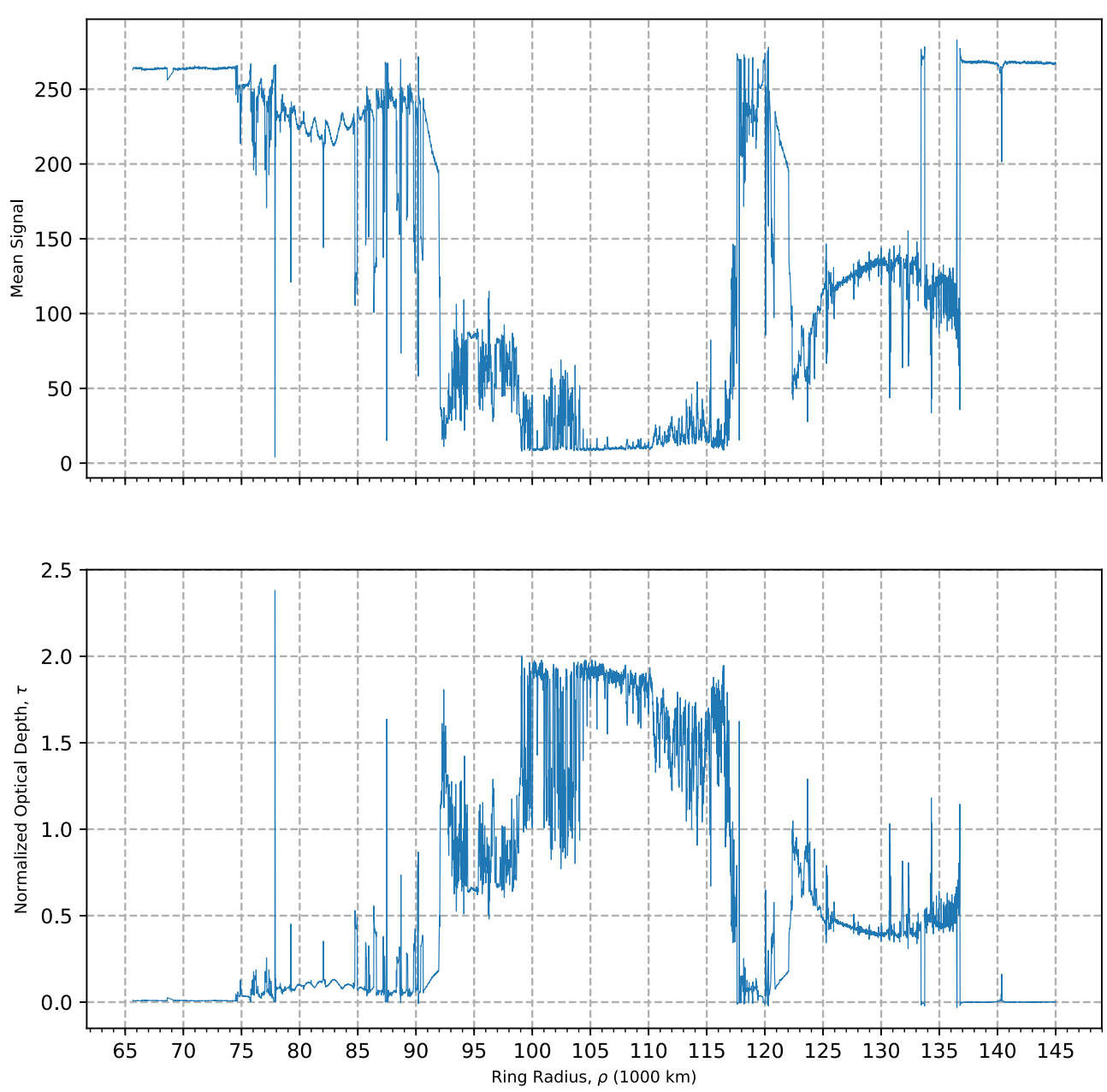

Fig. 17.- Ring profiles for the W Hya ingress occultation on rev 181, which provided a complete radial cut from the $\mathrm{F}$ ring to the $\mathrm{D}$ ring. The upper panel shows the measured light curve, in raw data numbers (DN), while the lower panel shows the corresponding normal optical depth profile, in the same format as Fig. 4. The data are summed over 8 spectral channels, centered at a wavelength of $2.92 \mu \mathrm{m}$, and are plotted at a radial resolution of $10 \mathrm{~km}$. The integration time was $20 \mathrm{msec}$ and the average range from Cassini to the rings was $690,000 \mathrm{~km}$, or $11.5 R_{S}$. The ring opening angle $\left|B_{*}\right|=34.64^{\circ}$. For this profile the maximum detectable normal optical depth should be 3.09, at the 3- $\sigma$ level, but because of a residual signal of $10 \mathrm{DN}$ in the $\mathrm{B}$ ring, the apparent value of $\tau$ does not exceed 2.0, even in the B3 region. 
due to problems with the reaction wheels or the onboard star-trackers.

Footnotes in Table 9 provide additional information specific to one or more events in Table 8. These include use of a nonstandard targeting vector; partial or complete loss of data due to pointing (four instances), DSN (two instances) or data-policing (two instances) problems; and 11 instances where the data quality is clearly affected by a bad stellar acquisition. Also noted are 11 events where there was a partial blockage or early termination of the ring occultation due to occultation of the star by Saturn, usually resulting in a loss of $\mathrm{C}$ and D ring data. Worthy of special note are the deep chord occultations on revs 194 (2 Cen) and 245 ( $\alpha$ Sco), each of which lost part of its $\mathrm{C}$ ring occultation to a grazing occultation by Saturn itself. Generally such overlapping atmospheric and ring occultations make both the ring and atmospheric data useless for detailed analysis.

More useful are those ring occultations that were followed by a non-overlapping Saturn ingress occultation for which VIMS atmospheric data were obtained. Sometimes the two occultations were combined in a single observation and at other times they were bookkept as separate observations. About 40 such serendipitous 'combined' occultations were observed, but many are of limited utility as Saturn occultations because either the star was seen against the planet's sunlit limb (resulting in a large non-stellar background signal) or the observations were made in spectrally-summed mode. The VIMS Saturn stellar and solar occultations will form the subject of a subsequent publication. In five cases, indicated by footnotes 3 or 22, a short VIMS occultation by the $\mathrm{C}$ ring was recorded as part of a planned Saturn occultation by either VIMS or UVIS; these events appear in the official Cassini lists of observations only as Saturn occultations, but some provided excellent $\mathrm{C}$ ring data.

Relevant geometric and photometric information for each successful occultation ${ }^{14}$ is given in Table 10 in the Appendix, including the star's saturnicentric latitude $B_{*}$ (extracted from Table 1), the average range $D$ from Saturn to Cassini and the resulting projected stellar diameter $D \theta_{*}$ and Fresnel zone diameter $\sqrt{2 \lambda D}$ and the star's radial velocity $v_{\text {rad }}$. Also listed here are the observed unocculted stellar signal $F_{0}$ and the predicted values of $\tau_{\min }$ and $\tau_{\max }$ at a resolution of $10 \mathrm{~km}$, calculated at the $3-\sigma$ level. As a rough rule-of-thumb, $\tau_{\max }$ decreases by $\sim \sin \left|B_{*}\right|$ for every factor-of-10 improvement in radial resolution, while $\tau_{\min }$ increases by a factor of 3 (see Eqns. (3) and (5)). To simplify this calculation, we have used the star's radial velocity in the middle A ring, at a radius of $125,000 \mathrm{~km}$. This is conservative, inasmuch as the velocity at smaller radii is generally less, resulting in more samples per $\mathrm{km}$ and thus smaller values of $\tau_{\min }$ and larger values of $\tau_{\max }$. In the VIMS ring occultation files delivered to the PDS, however, the local value of $v_{\text {rad }}$ is used at each radius. As result, the values given there for $\tau_{\min }$ and $\tau_{\max }$ differ slightly from those listed in Table 10.

Chord occultations are of particular interest because they afford exceptionally high radial sampling in the vicinity of the turn-around point. The final column in Table 8 lists

\footnotetext{
${ }^{14}$ Note that this table contains only 182 entries, rather than 190 , due to the omission of the eight occultations for which no useful data were returned.
} 
the general locations of turn-around radii for chord occultations, while footnotes identify four instances in which the turn-around point falls within or immediately interior to a narrow gap. Table 11 lists the turnaround radii for each of the 74 chord occultations in Table 8 , accurate to about $1 \mathrm{~km}$, along with the radial velocity at our standard radius of $125,000 \mathrm{~km}$. (The radial velocity at the turnaround radius is, of course, zero.) Some of these chord occultations may prove to be fruitful subjects for a future investigation. As an example, we note the study by Hedman et al. (2014) of a region of viscous overstability in the inner A ring using the $\gamma$ Cru chord occultations on revs 104 and 106.

Appendix B contains additional information on some of the more interesting VIMS ring occultations, in particular those that form part of an extended series of observations.

\section{Solar occultations}

\subsection{Instrumental considerations}

Both the VIS and IR channels of the VIMS instrument are also equipped with solar ports, which permit off-axis observations of the Sun. Originally intended for purposes of spectral and flux calibration, the IR solar port has also proven to be usable for observing solar occultations by the rings, Saturn and Titan. As described by Brown et al. (2004), the IR solar port consists of a small off-axis aperture within the primary telescope that intercepts $\sim 0.4 \%$ of the incident sunlight, and then passes this through a chain of six $45^{\circ}$ prisms made of $\mathrm{ZnS}$ which further attenuate the signal by a factor of $1 \times 10^{-4}$. The resulting flux, strongly polarized and attenuated by an overall factor of $4 \times 10^{-7}$, is then passed through the VIMS telescope and spectrometer optics in the same fashion as a normal target scene. ${ }^{15}$ In order to match the alignment of the solar port in the UVIS instrument, and to avoid damaging other sensitive optical systems on Cassini by directly observing the Sun, the VIMS solar port looks out in a direction $20^{\circ}$ away from the main instrument boresight (which is oriented along the spacecraft's $-\mathrm{Y}$ axis), offset towards the high gain antenna ( $\mathrm{Z}$ axis).

In the course of early solar observations, it was discovered that the boresights of the VIMS and UVIS solar ports are actually offset by $12.0 \mathrm{mrad}$, or approximately one-third of the VIMS FOV. In order to accommodate simultaneous UVIS observations, most subsequent VIMS ring solar occultations were observed using the UVIS boresight, so that the solar image appears towards one edge of the full VIMS FOV, as illustrated in Fig. 21 below.

\footnotetext{
${ }^{15}$ The solar port in the VIS channel works, in part, by diffusing the solar image along the instrument's spectrometer slit. This, combined with partial saturation of the solar spectrum, unfortunately makes it impossible to make quantitative measurements of the solar flux at visual wavelengths.
} 


\subsection{Spatial resolution}

Unlike the situation with most stellar occultations, the spatial resolution of solar occultations is usually set by the finite solar angular diameter rather than by the sampling time. At Saturn's mean distance from the Sun of $9.5 \mathrm{AU}$, the solar diameter $\theta_{\odot} \simeq 1.0 \mathrm{mrad}$, or 2 standard VIMS pixels. For typical ring solar occultations, the spacecraft-target distance $D \simeq 400,000 \mathrm{~km}$ results in a projected solar diameter $D \theta_{\odot} \simeq 400 \mathrm{~km}$, give or take a factor of 2. The large projected size of the solar disk means that the rapid fluctuations in signal characteristic of ring stellar occultations are not seen in solar occultations. Indeed, the characteristic minimum time scale for significant signal variations is of order $t_{\odot}=D \theta_{\odot} / v$, where $v$ is the transverse component of the relative velocity of Cassini and the target body. For typical ring solar occultations $v \simeq 10 \mathrm{~km} \mathrm{~s}^{-1}$, so that we have $t \odot \simeq 40 \mathrm{sec}$.

Because the Sun is not a point source as seen by VIMS, solar occultations cannot be observed in single-pixel mode as are stellar occultations. Our approach, therefore, is to observe solar occultations by obtaining a continuous series of small IR cubes of the Sun, from which we can later estimate the solar flux as a function of time and wavelength. In most cases an image size of $8 \times 8$ pixels has proven to be satisfactory, with an integration time of 40 or $60 \mathrm{msec}$. (Longer times lead to saturation of the detector at shorter wavelengths.) A single cube is acquired in 4-6 sec, including the necessary background measurements, which is more than sufficient given the above estimate of $t_{\odot}$.

\subsection{Summary of observations}

Over the course of the Cassini mission, VIMS has successfully observed 30 solar occultations by Saturn's rings, covering the low-optical-depth D, F and G rings as well as the main $\mathrm{A}, \mathrm{B}$ and $\mathrm{C}$ rings. One observation was lost due to problems acquiring the communications signal at the Deep Space Network receiving station. As for the stellar occultations, the geometry of these observations includes both simple radial profiles of the entire ring system and chord occultations of various depths. Because star-finding cubes are unnecessary for solar occultations, both ingress and egress events have been observed with comparable frequency, along with a number of partial occultations whose timing was primarily dictated by other experiments. A combination of $8 \times 8$ and $12 \times 12$ pixel cube sizes has been used, as well as $24 \times 24,32 \times 32$ and even $64 \times 64$ cubes in a few instances (see below). A complete list of ring solar occultations observed by VIMS is provided in Table $\mathbf{6}$, including the orbit number, start time, duration, ring opening angle to the sun, $\left|B_{\odot}\right|$, Cassini's distance to Saturn, $D$, and observing mode. A brief description of the radial coverage of each occultation is provided in the 'Notes' column. Additional descriptive notes are provided in Table 7.

Fig. 18 shows an example of a complete egress radial profile across the main rings, at a wavelength of $2.26 \mu \mathrm{m}$, where we see first the semi-transparent $\mathrm{C}$ ring, with several partially-resolved plateaux and narrow gaps, followed in turn by the almost-opaque B ring, the Cassini Division and the intermediate optical depth A ring. Note the sharp inner and outer edges of the A and B rings, as well as the Encke gap in the outer A ring, whose width 
Table 6: List of all Cassini-VIMS ring solar occultations.

\begin{tabular}{|c|c|c|c|c|c|c|c|c|c|}
\hline $\operatorname{Rev}^{a}$ & $\begin{array}{c}\text { Start time } \\
\text { (year-dayThh:mm) }\end{array}$ & $\begin{array}{l}\left|B_{\odot}\right|^{b} \\
\text { (deg) }\end{array}$ & $\begin{array}{r}\mathrm{D}^{c} \\
(\mathrm{Mm})\end{array}$ & $\begin{array}{l}\text { Duration } \\
\text { (hh:mm) }\end{array}$ & Mode $^{d}$ & $\begin{array}{r}\tau_{\mathrm{IR}}^{e} \\
(\mathrm{~ms})\end{array}$ & Seq. & Ptg. ${ }^{f}$ & Notes $^{g}$ \\
\hline 9 & 2005-159T11:30 & $21.45^{\circ}$ & 245 & $2: 40$ & $12 \times 12$ & 40 & S11 & $\mathrm{P} 0$ & F-C rings $(\mathrm{I})^{2}$ \\
\hline $11^{4}$ & 2005-196T00:26 & $21.07^{\circ}$ & 250 & $1: 20$ & $12 \times 12$ & 40 & S12 & $\mathrm{P} 2$ & $\mathrm{~B}-\mathrm{C}$ rings $(\mathrm{I})^{2,5}$ \\
\hline 28 & 2006-257T19:22 & $15.86^{\circ}$ & 2053 & 11:08 & $32 \times 32$ & 40 & S23 & $\mathrm{P} 1$ & $\mathrm{~A}-\mathrm{C}$ rings $(\mathrm{I})^{1}$ \\
\hline 43 & 2007-114T09:45 & $12.77^{\circ}$ & 452 & $2: 30$ & $12 \times 12$ & 40 & S29 & $\mathrm{P} 1$ & ABF chord $^{6}$ \\
\hline 55 & 2008-003T20:00 & $9.00^{\circ}$ & 285 & $1: 45$ & $12 \times 12$ & 40 & S36 & $\mathrm{P} 1$ & FBF chord \\
\hline 59 & 2008-051T16:31 & $8.27^{\circ}$ & 220 & $0: 55$ & $12 \times 12$ & 40 & S38 & $\mathrm{P} 1$ & $\mathrm{~F}-\mathrm{C}$ rings $(\mathrm{I})^{2}$ \\
\hline 62 & 2008-083Т08:45 & $7.79^{\circ}$ & 247 & $2: 00$ & $12 \times 12$ & 40 & S39 & $\mathrm{P} 1$ & FCF chord \\
\hline 65 & 2008-111T20:05 & $7.36^{\circ}$ & 279 & $2: 10$ & $12 \times 12$ & 40 & S40 & $\mathrm{P} 1$ & FCF chord $^{7}$ \\
\hline 66 & 2008-121T09:10 & $7.21^{\circ}$ & 278 & $2: 10$ & $12 \times 12$ & 40 & & $\mathrm{P} 1$ & FCF $_{\text {chord }}{ }^{7}$ \\
\hline 85 & 2008-261T16:00 & $5.05^{\circ}$ & 242 & $1: 45$ & $12 \times 12$ & 60 & S44 & $\mathrm{P} 2$ & $\mathrm{FCF}_{\text {chord }}{ }^{7}$ \\
\hline 90 & 2008-298T08:57 & $4.49^{\circ}$ & 241 & $1: 37$ & $12 \times 12$ & 60 & S45 & P2 & FBF chord $^{8}$ \\
\hline 172 & 2012-267T22:43 & $15.94^{\circ}$ & 596 & $2: 07$ & $8 \times 8$ & 40 & S75 & $\mathrm{P} 0$ & $\mathrm{~F}-\mathrm{C}$ rings (I) \\
\hline 181 & 2013-044T22:50 & $17.54^{\circ}$ & 563 & $1: 12$ & $12 \times 12$ & 40 & S77 & $\mathrm{P} 0$ & $\mathrm{~B}-\mathrm{F}$ rings $(\mathrm{E})$ \\
\hline $239 \mathrm{i}$ & 2016-221T21:50 & $26.39^{\circ}$ & 808 & 1:01 & $8 \times 8$ & 60 & S95 & $\mathrm{P} 1$ & $\mathrm{~F}-\mathrm{A}$ rings $(\mathrm{I})^{14}$ \\
\hline $239 \mathrm{e}$ & 2016-222Т02:27 & $26.39^{\circ}$ & 838 & $3: 33$ & $8 \times 8$ & 60 & & $\mathrm{P} 1$ & D-F rings $(E)^{3,14}$ \\
\hline 241 & 2016-245T $17: 48$ & $26.45^{\circ}$ & 818 & $6: 14$ & $8 \times 8$ & 60 & S95 & $\mathrm{P} 1$ & FCA chord $^{9}$ \\
\hline 243 & 2016-269T19:20 & $26.50^{\circ}$ & 842 & $2: 49$ & $8 \times 8(S)$ & 50 & S96 & $\mathrm{P} 1$ & $\mathrm{D}-\mathrm{B}$ rings $(\mathrm{E})^{9}$ \\
\hline 245 & 2016-288T16:30 & $26.53^{\circ}$ & 765 & $4: 36$ & $8 \times 8(S)$ & 60 & & $\mathrm{P} 1$ & 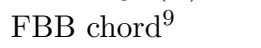 \\
\hline 249 & 2016-325T05:09 & $26.59^{\circ}$ & 725 & $6: 30$ & $8 \times 8(S)$ & 60 & & $\mathrm{P} 1$ & FBF chord \\
\hline 254 & 2016-361T16:24 & $26.64^{\circ}$ & 607 & $8: 03$ & $8 \times 8(S)$ & 60 & S97 & $\mathrm{P} 1$ & FCF chord \\
\hline 257 & 2017-017T04:00 & $26.66^{\circ}$ & 597 & $1: 32$ & $8 \times 8$ & 60 & S97 & $\mathrm{P} 1$ & F-B rings $(\mathrm{I})^{12}$ \\
\hline 260 & 2017-038T21:40 & $26.68^{\circ}$ & 710 & $3: 42$ & $24 \times 24(\mathrm{~S})$ & 60 & S98 & $\mathrm{P} 1$ & D-F rings $(E)^{14}$ \\
\hline 261 & 2017-046T02:32 & $26.69^{\circ}$ & 725 & $2: 58$ & $8 \times 8(\mathrm{~S})$ & 60 & & $\mathrm{P} 1$ & D-F rings $(\mathrm{E})$ \\
\hline 262 & 2017-053Т06:47 & $26.70^{\circ}$ & 698 & $2: 40$ & $8 \times 8(S)$ & 60 & & $\mathrm{P} 1$ & $\mathrm{D}-\mathrm{F}$ rings $(\mathrm{E})^{14}$ \\
\hline 263 & 2017-060T11:10 & $26.70^{\circ}$ & 704 & $1: 34$ & $24 \times 24$ & 60 & & $\mathrm{P} 1$ & $\mathrm{C}-\mathrm{B}$ rings $(\mathrm{E})^{12,14}$ \\
\hline 265 & 2017-074T19:29 & $26.71^{\circ}$ & 713 & $2: 15$ & $8 \times 8$ & 60 & S98 & $\mathrm{P} 1$ & $\mathrm{~B}-\mathrm{F}$ rings $(\mathrm{E})^{12,14}$ \\
\hline 267 & 2017-089T03:38 & $26.72^{\circ}$ & 729 & $1: 50$ & $8 \times 8$ & 60 & & $\mathrm{P} 1$ & $\mathrm{~B}-\mathrm{F}$ rings $(\mathrm{E})^{12}$ \\
\hline 269 & 2017-103Т04:10 & $26.73^{\circ}$ & 583 & $1: 13$ & $24 \times 24(\mathrm{~S})$ & 60 & & $\mathrm{P} 1$ & $\mathrm{~F}-\mathrm{A}$ rings $(\mathrm{I})^{12,14}$ \\
\hline 271 & 2017-116T21:20 & $26.73^{\circ}$ & 558 & $1: 40$ & $24 \times 24(\mathrm{~S})$ & 60 & S99 & $\mathrm{P} 1$ & $\mathrm{~A}-\mathrm{G}$ rings $(\mathrm{E})^{12,14}$ \\
\hline 277 & 2017-155T07:42 & $26.73^{\circ}$ & 440 & $5: 40$ & $64 \times 64(\mathrm{~S})$ & 60 & S100 & $\mathrm{P} 1$ & No data ${ }^{13,15}$ \\
\hline $279^{4}$ & 2017-168T05:30 & $26.73^{\circ}$ & 438 & $5: 55$ & $64 \times 64(\mathrm{~S})$ & 60 & S100 & $\mathrm{P} 1$ & ACA chord $^{5,15}$ \\
\hline
\end{tabular}

${ }^{a}$ Cassini orbit number.

${ }^{b}$ Ring opening angle to the sun.

${ }^{c}$ Cassini's mean distance from Saturn.

${ }^{d}$ Cube size; $\mathrm{S}=$ spectrally-summed.

${ }^{e}$ Integration time.

${ }^{f}$ Spacecraft pointing vector used to target the sun: $\mathrm{P} 0=$ VIMS-IR-SOL, P1 $=$ UVISSOLAR, P2 = other nonstandard vector.

${ }^{g}$ Occultation coverage and geometry: $\mathrm{I}=$ ingress, $\mathrm{E}=$ egress. See Table 7 for numbered footnotes. 
of $325 \mathrm{~km}$ is just slightly less than that of the projected solar disk of $\sim 360 \mathrm{~km}$. Outside the $\mathrm{A}$ ring the narrow $\mathrm{F}$ ring is visible, though unresolved.

Fig. 19 illustrates a typical chord occultation, in this case at a wavelength of $0.94 \mu \mathrm{m}$, which shows similar structure in the A and B rings, but with a significant variation in the transmission through the A ring between ingress and egress due to the presence of strong self-gravity wakes in this region. This particular observation is the second-longest solar occultation recorded by Cassini, with over 5400 cubes being acquired over an $8 \mathrm{hr}$ period. The sun reached its minimum apparent distance from Saturn in the central C ring, $\sim 4 \mathrm{hrs}$ after the start of observations.

The slowest solar occultation observed was the very distant ingress event on rev 28, which occurred over a period of $24 \mathrm{hrs}$. Because of overlap with a higher-priority radio occultation by the rings, VIMS was only able to observe the central portion of this unique event, covering the inner $\mathrm{A}$ ring, $\mathrm{B}$ ring and outer $\mathrm{C}$ ring. Because of the very large range of over $2 \times 10^{6} \mathrm{~km}$, the spatial resolution of this data set is $\sim 1000 \mathrm{~km}$.

\subsection{Photometric calibration}

The optical design of the solar port, with its series of multiple internal reflections, results in a significant background of scattered light across the instrument's FOV. Furthermore, the spectrum of the sun depends, to some degree, on where its image falls within the FOV. ${ }^{16}$ Both effects are illustrated in Fig. 20, where representative solar and background spectra are shown for the sun in the upper, middle and lower parts of the VIMS FOV. Fortunately the solar spectrum is found to be a slowly-varying function of position in both the $\mathrm{X}$ and $\mathrm{Z}$ directions, with the main variations occurring at wavelengths shortward of $\sim 1.6 \mu \mathrm{m}$. The scattered light background peaks at around $2.3 \mu \mathrm{m}$, where it amounts to $>10 \%$ of the peak flux in the main solar image, but its spectrum is relatively insensitive to the position of the sun in the field. Its absolute level does, however, vary somewhat with the position of the sun in the instrument's FOV.

We have obtained consistent and reproducible photometric light curves for ring solar occultations with two methods: (1) fitting a 2D gaussian function to each cube, channel by channel, to determine the peak height and width of the solar image, from which the total direct flux from the sun can be estimated, and (2) simply co-adding the total flux, both direct and scattered, in each cube for each channel. As long as the pointing and cube size do not change during the occultation, both methods yield consistent and essentially identical results, but the statistical fluctuations are less for the second method, which we use for the results shown here. In a typical $8 \times 8$ cube, with an integration time of $50 \mathrm{~ms}$ per pixel, the total signal recorded for the unoccculted sun at a wavelength around $2.3 \mu \mathrm{m}$ is $\sim 1.9 \times 10^{4} \mathrm{DN}$

\footnotetext{
${ }^{16}$ This is due to the fact that the small aperture of the solar port does not fill the entrance pupil of the spectrometer optics, which results in an uneven illumination of the multiple-blaze diffraction grating when compared with that of the full aperture.
} 
Rev 261 SOLAROCC

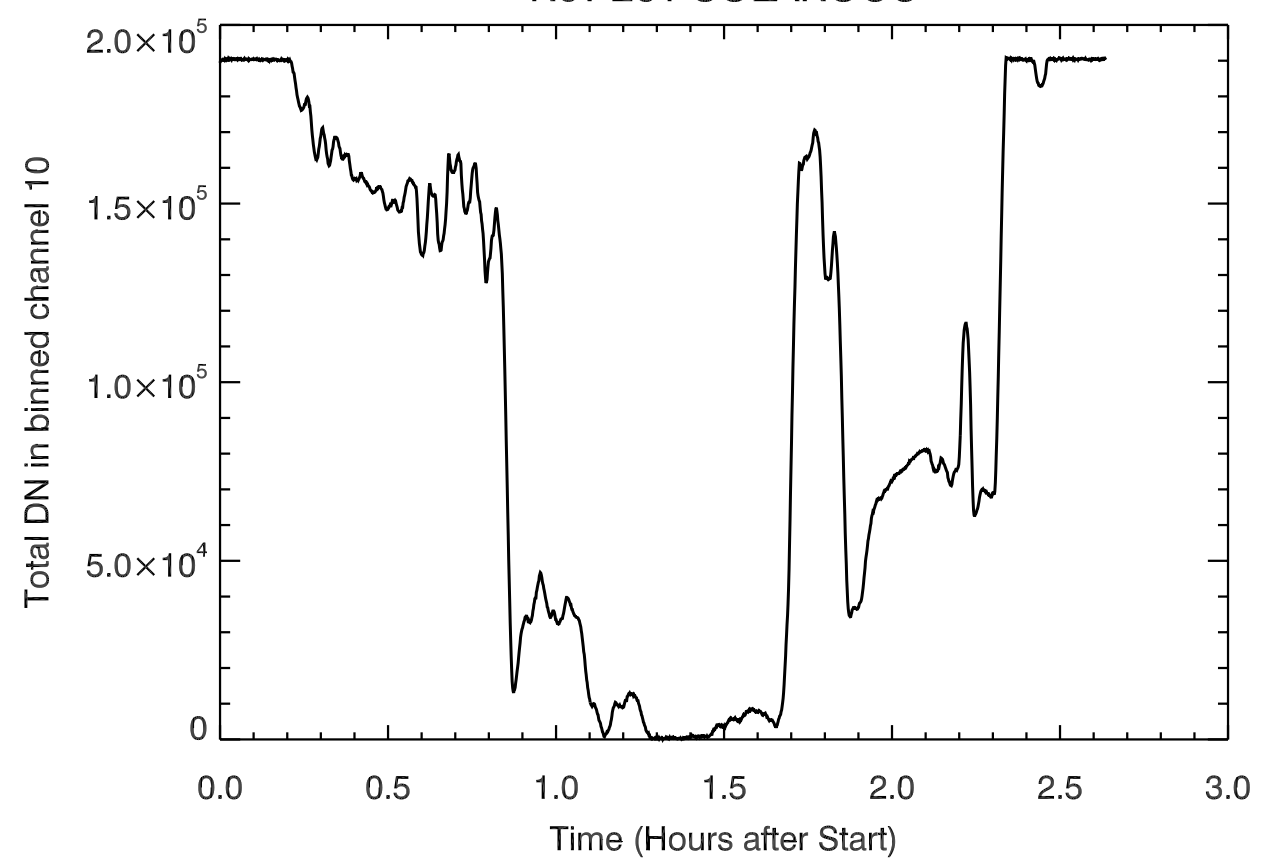

Fig. 18. - A complete egress radial solar occultation profile of the rings obtained by Cassini VIMS on rev 261, displayed here at a wavelength of $2.26 \mu \mathrm{m}$ and plotted vs observation time. The integration time was $60 \mathrm{~ms}$ per pixel and the data have been summed over 8 spectral channels to reduce data volume. The latitude of the sun was $B_{\odot}=26.69^{\circ}$ and the average range to Saturn was 725,000 km, resulting in a radial resolution of $\sim 360 \mathrm{~km}$. 


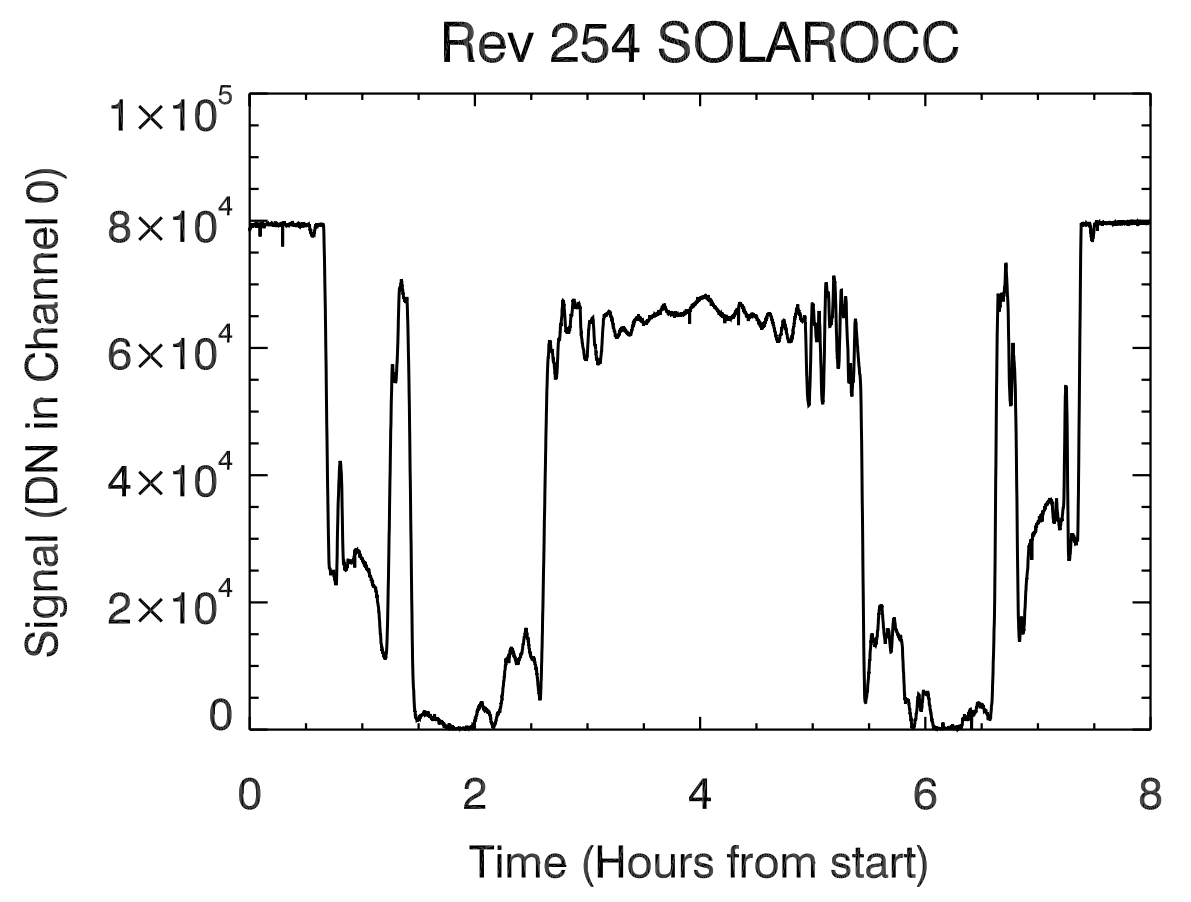

Fig. 19.- A solar occultation profile of the rings obtained by Cassini VIMS on rev 254, corresponding to a chord across the $\mathrm{A}, \mathrm{B}$ and $\mathrm{C}$ rings. The data are displayed here at a wavelength of $0.94 \mu \mathrm{m}$ and plotted vs observation time. The integration time was $60 \mathrm{~ms}$ per pixel and the data have been summed over 8 spectral channels to reduce data volume. The range here was $607,000 \mathrm{~km}$, but the radial resolution varies as the orientation of the projected solar disk changes across the chord. In this case, it is better on egress. The latitude of the sun was $B_{\odot}=26.64^{\circ}$. 
per channel, of which $\sim 6500 \mathrm{DN}$ is from the direct solar image with the remainder coming from the integrated scattered light background $(8 \times 8 \times 190 \simeq 12,200 \mathrm{DN}$, using the numbers from Fig. 20). For spectrally-summed observations, such as those in Figs. 18 and 19, these levels are increased by a factor of 8 to an overall signal of $\sim 1.5 \times 10^{5} \mathrm{DN}$.

The statistical noise level in solar occultation data is again due a combination of detector read noise, $N_{r}$ and shot noise from the direct solar signal, $N_{\odot}$ and from the scattered light background, $N_{s}$. For $8 \times 8$ cubes, spectrally-summed over 8 channels, the former is approximately $\sqrt{8^{3}}=22.6 \mathrm{DN}$, based on a detector read noise of $1 \mathrm{DN}$ per pixel per channel. Using the same expressions developed in Section 6.4, and the flux levels in Fig. 20, we find that $N_{\odot} \simeq \sqrt{6500 \times 8 / 350}=12.2 \mathrm{DN}$, while $N_{s} \simeq \sqrt{190 \times 64 \times 8 / 350}=16.7 \mathrm{DN}$. The shot noise associated with the variable part of the instrument background (see Sec 6.4 ) is $\sim 3 \mathrm{DN}$ and negligible. The overall noise level per measurement is thus $\sim 30.6 \mathrm{DN}$, corresponding to an unocculted SNR of $\sim 4900$, or $\sim 10$ times higher than that for the best stellar occultations, albeit at much lower radial resolution.

Using the expressions for the minimum and maximum detectable optical depth given in Sections 6.5 and 6.6, and the above numerical values for a spectrally-summed $8 \times 8$ cube with an integration time of $50 \mathrm{~ms}$ per pixel, we find that $\tau_{\min } \simeq 0.00061 \sin \left|B_{\odot}\right|$ and $\tau_{\max } \simeq 7.7 \sin \left|B_{\odot}\right|$, per cube, both at the $3-\sigma$ level. (As noted above, such a cube is acquired every $4-6 \mathrm{sec}$, or roughly every $50 \mathrm{~km}$ for a typical solar occultation.) This estimate for $\tau_{\min }$ is similar to those for the best stellar occultations (see Table 4), especially for the lower-inclination solar occultations. However, the maximum possible inclination for solar occultations of $\left|B_{\odot}\right|=26.7^{\circ}$ limits $\tau_{\max }$ to $\sim 3.5$, substantially lower than the best stellar occultations (see Table 5). In practice, given the effective radial resolution for the solar occultation data of $\sim 400 \mathrm{~km}$, the measured solar flux can be averaged over 8-10 cubes and $\tau_{\max }$ improved to $\sim 4.0$.

Because the VIMS solar port utilizes the same optics and focal-plane shutter as does the main IR channel, both are in fact 'active' at all times. The strong attenuation in the solar port ensures that the sun is the only target capable of producing a measurable signal when observed through the solar port, but if another target happens to cross the FOV of the main aperture during a solar occultation then the combined signals of both are recorded by the VIMS focal plane. An example of such a circumstance is shown in Fig. 21, where during a ring solar occultation on rev 279 the main aperture crossed the opposite ring ansa. The result is a faint image of the middle $\mathrm{C}$ ring superimposed on that of the sun. Although the presence of the (moving) ring background made it impossible to obtain a useful occultation lightcurve from this observation, it did offer an opportunity to obtain an unusual series of images of the full IR FOV as observed through both the main VIMS aperture and the solar port. We see not only the bright (saturated in this stretch) main image of the sun in the upper part of the frame, centered on the UVIS solar port boresight at pixel [36,53], but also multiple secondary images produced by internal reflections within the solar port itself. 

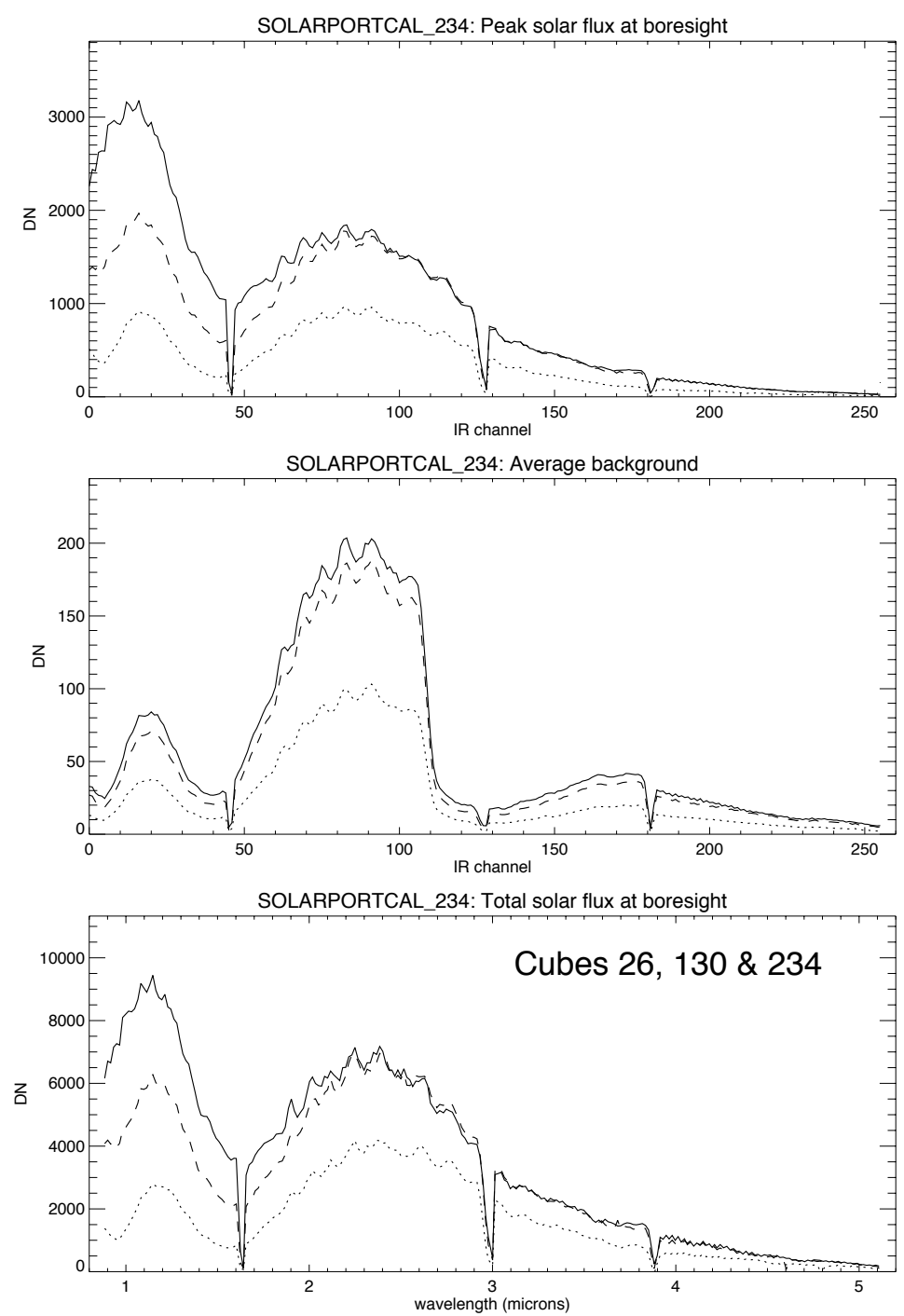

Fig. 20. - Spectra of the sun obtained through the VIMS-IR solar port during a calibration observation on rev 234 in which the solar image was moved systematically across the VIMS FOV in a raster-scan pattern. For each cube, the image of the sun was fitted by a $2 \mathrm{D}$ gaussian function to determine the peak flux, mean background signal, image centroid and FWHM image size, all as functions of wavelength. The top panel shows the peak solar flux spectrum for three positions of the sun: at pixel $[28,10]$ (dotted curve), pixel $[31,30]$ (dashed curve) and pixel [35,49] (solid curve). The center panel shows the mean background spectrum (in DN per pixel) for the same three cubes. The lower panel shows the total solar flux spectrum for the same cubes, obtained by integrating over the solar image after subtracting the mean background level. Sharp dips correspond to gaps between segments in the VIMS focal plane blocking filter. The integration time was $50 \mathrm{~ms}$ per pixel and the cube size was $56 \times 16$ pixels. 


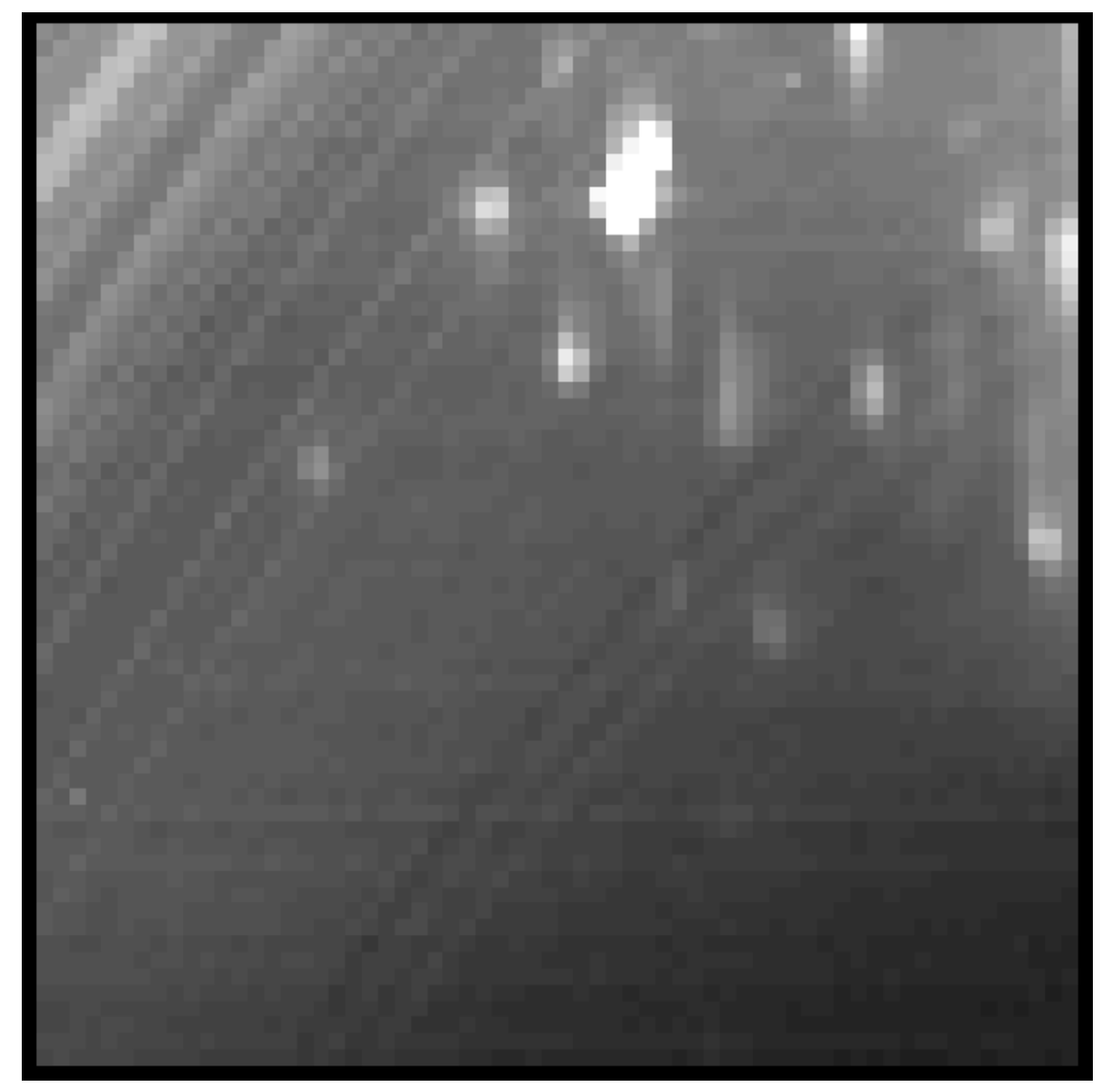

Fig. 21.- An image obtained during a solar occultation of the rings observed by Cassini VIMS on rev 279, using the instrument's full $64 \times 64$ field of view. In this case, the main aperture captured an image of the $\mathrm{C}$ ring at the same time as the solar port was observing the sun, on the opposite ansa (see text). The data displayed here are for a wavelength of $1.07 \mu \mathrm{m}$ and the integration time was $60 \mathrm{~ms}$ per pixel. The range to the rings was $438,000 \mathrm{~km}$. As in Fig. 1, the image is displayed with $\mathrm{Z}$ increasing upwards and $\mathrm{X}$ increasing towards the right. The main solar image is centered at pixel [36,53], the location of the UVIS solar port boresight. It is saturated in this stretch, in order to show the much fainter secondary images due to scattering in the solar port optics. 


\subsection{Applications}

Solar occultations by the rings have so far proven to be useful primarily in placing constraints on the particle size distribution in the rings via their forward-scattered radiation at both near-infrared and ultraviolet wavelengths (Harbison et al. 2013; Becker et al. 2018). It is to this end that larger image sizes were used for some later ring solar occultations. However, the presence of secondary reflections in the VIMS solar port images, as illustrated in Fig. 21, along with the smooth halo of diffusely-scattered light that extends across the entire FOV, significantly complicates these efforts (Harbison et al. 2013).

Because of their extremely high signal-to-noise ratio, solar occultations provide the most accurate estimates of the mean transmission of broad regions of the rings as a function of wavelength in the near-infrared. As seen in Fig. 22, the main rings are found to be spectrally 'gray' in transmission, with no detectable variations in optical depth across the 1-5 $\mu \mathrm{m}$ range (Harbison et al. 2013). This is consistent with an almost complete absence of free-floating ring particles smaller than a few $\mathrm{mm}$ in size, as any appreciable population of such particles would lead to water ice absorption bands in the solar port spectra. (An exception to this generalization is the narrow, dusty F ring, where both stellar occultation and high-phase imaging data show strong signatures of small water ice grains at $2.87 \mu \mathrm{m}$ (Hedman et al. 2011; Vahidinia et al. 2011).)

\section{Discussion}

Occultation observations provide the highest-resolution probes of planetary ring systems and have proved to be invaluable in unravelling many of the dynamical puzzles surrounding Saturn's rings, as well as providing much indirect information on the size distribution and spatial arrangement of the millimeter-to-meter size particles which make up the main rings. A major contribution to the existing occultation data set for Saturn's rings is the body of data acquired by the VIMS instrument during Cassini's 13-year orbital mission, complementing similar data acquired at ultraviolet and microwave wavelengths by the UVIS and RSS experiments. Although not envisioned in the original design of the instrument, VIMS has proven to be a versatile and effective platform for observing both stellar and solar occultations by Saturn's rings. This is attributable, in part, to the intrinsic flexibility of the instrument, with its adjustable pixel size, image dimensions, and spectral resolution, as well as to software upgrades carried out after Cassini's launch in 1997. The latter include the capability to carry out autonomous on-board stellar acquisition, spectral summing and editing, and to record the observation times of individual spectra. Also greatly beneficial to the successful use of VIMS as an occultation instrument is its photometric linearity, relatively low thermal background and dark current (making background subtraction much more straightforward than for some infrared instruments) and lack of hysteresis. Finally, we should note that the acquisition and high-speed observation of stars with what is fundamentally an imaging instrument rather than a photometer has been possible only because of the Cassini spacecraft's extremely precise pointing and tracking capabilities. 


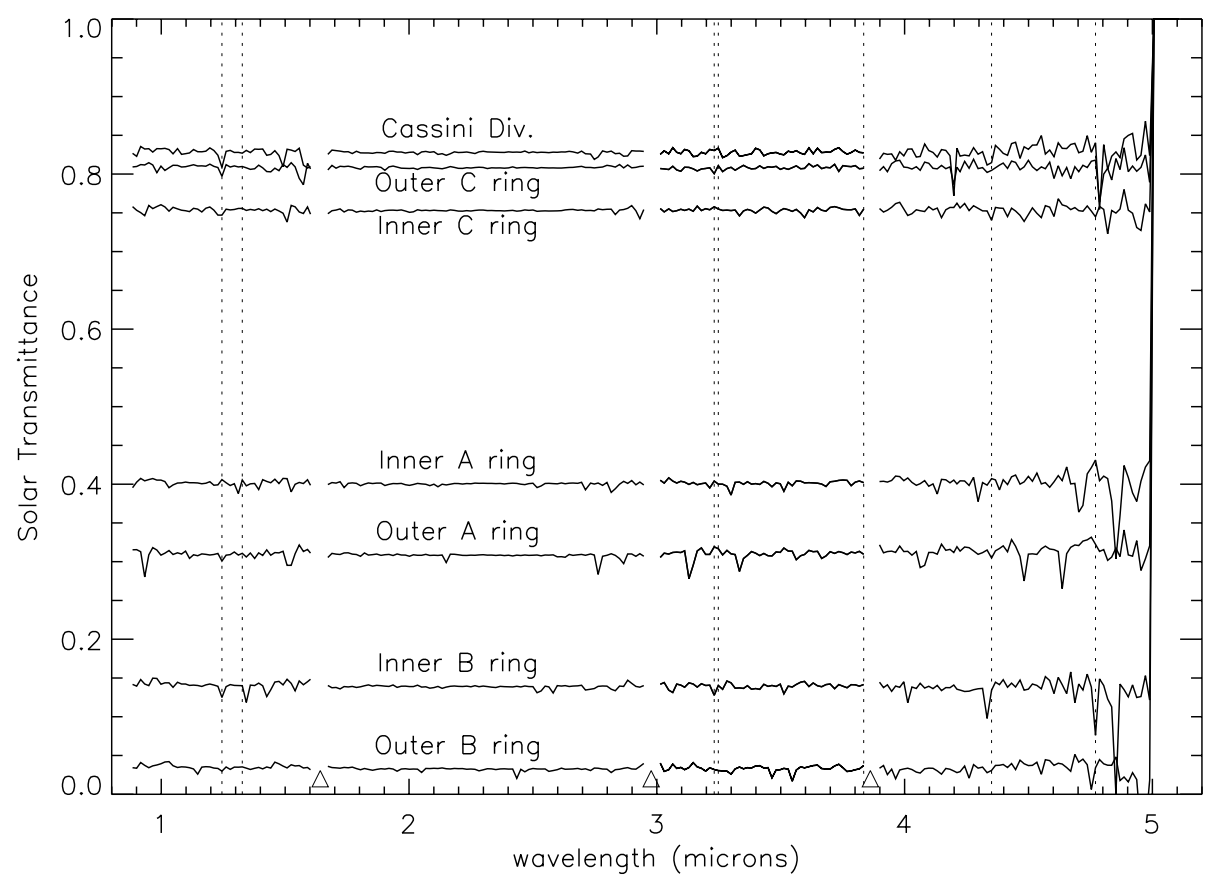

Fig. 22.- A series of transmission spectra of the main rings derived from a solar occultation observed by Cassini VIMS on rev 9. Shown is the ratio of the solar spectrum, as seen through seven different regions of the rings, ratioed to the average solar spectrum observed before the occultation began. Triangles indicates gaps in the order-sorting focal plane filter, while vertical dotted lines indicate unreliable or "hot" pixels which can sometimes saturate. The latitude of the sun was $B_{\odot}=-21.45^{\circ}$ and the range to Saturn was $245,000 \mathrm{~km}$. 
In this paper we have emphasized the planning, design and execution of both stellar and solar ring occultations with VIMS, and discussed in some detail the geometric and photometric calibration of the data. Uncertainties in the geometric reconstruction and photometry are examined, and the minimum and maximum optical depths detectable are estimated for each of our target stars. Tables 6, 8, 10 and 11 summarize the complete VIMS ring occultation data set and provide a compact source of information for future researchers wishing to make use of the data. The following is a summary of our principal findings.

- During the course of Cassini's orbital tour, the VIMS instrument observed almost 200 stellar occultations by the rings, as well as 30 solar occultations. The stellar data span the period May 2005 - September 2017 (revs 8 - 292) but with significant gaps in 2005/6, 2007, 2010-12 and 2015 when the spacecraft was in near-equatorial orbits. The stellar data were almost all obtained in single-pixel, spectrally-summed mode where the IR data are reduced from 248 to 31 spectral channels, each $\sim 0.14 \mu \mathrm{m}$ in width (see Table 8). Durations of individual occultations ranged from $1 \mathrm{hr}$ to just over $24 \mathrm{hrs}$, but only rarely exceeded 8 hrs.

- VIMS solar occultation data span almost the full range of solar inclination angles, from a minimum of $\left|B_{\odot}\right|=4.5^{\circ}$ in Oct 2008 to a maximum of $26.7^{\circ}$ in 2017 , near the northern winter solstice (see Table 6). Solar occultations have been obtained both at full spectral resolution (248 channels at $\delta \lambda \simeq 0.017 \mu \mathrm{m})$ and in spectrallysummed mode. All were observed in imaging mode, with cube sizes varying from $8 \times 8$ to $64 \times 64$ (see Table 6 ). Durations ranged from $1 \mathrm{hr}$ to just over $11 \mathrm{hrs}$ for one particularly distant event.

- Particularly useful for dynamical studies of noncircular features and externally-driven density waves in the rings are several series of occultations performed with the same star over relatively short periods of time. These include a series of 17 occultations of $\gamma \mathrm{Cru}$ in 2008/9, seven occultations of $\alpha$ Sco in 2016, eleven occultations of $\alpha$ Ori in 2016/7 and a final set of eight occultations of $\gamma \mathrm{Cru}$ in 2017. The $\gamma \mathrm{Cru}$ occultations are almost all radial cuts across the $\mathrm{A}, \mathrm{B}$ and $\mathrm{C}$ rings, whereas the $\alpha$ Ori occultations are mostly chords of various depths. Both the latter and the $\alpha$ Sco occultations are especially well-suited to studies of structure in low-optical depth regions such as the $\mathrm{C}$ ring and Cassini Division, while $\gamma$ Cru has provided our best data on the opticallythick parts of the B ring. Also notable is a set of four low-inclination chord occultations of o Ceti in 2005, though these sampled only the F ring, A ring and (once) the Cassini Division.

- Over $80 \%$ of the stellar occultations, and all but one of the solar occultations, led to data of useful quality. Of the remaining observations, $\sim 11 \%$ suffer from low signal levels and/or variable baselines due to poor pointing, while another $4 \%$ were lost completely due to a failure to acquire the star, Deep Space Network (DSN) communication problems, or data-policing. About $3 \%$ of the occultations suffered a partial loss of coverage due primarily to data drop-outs at the DSN station. 
- The radial resolution of the stellar occultation profiles ranges from $\sim 50 \mathrm{~m}$ to $\sim 500 \mathrm{~m}$, depending on the stellar angular diameter, spacecraft range, and sampling interval (see Table 10 and Fig. 7). For solar occultations the resolution is set by the projected size of the solar disk, and is typically $\sim 400 \mathrm{~km}$.

- Global geometric reconstruction of the occultation tracks is found to be accurate to $\sim 1 \mathrm{~km}$, limited by the accuracy of Cassini's trajectory. Local trajectory corrections, however, based on the known radii of quasi-circular features, yield radii that are selfconsistent to within $\sim 150 \mathrm{~m}$ (French et al. 2017).

- Although stellar occultations seen against the sunlit rings have a substantial component of non-stellar background flux, this is greatly reduced at longer wavelengths, where the reflectivity of water ice is quite low. Our primary light-curves are based on data acquired at a wavelength of $2.92 \mu \mathrm{m}$, where the rings are almost black, even in direct sunlight, and no background correction is necessary in most cases.

- Solar occultation data demonstrate that the optical depth of the main rings is spectrally neutral in the near-IR region observed by VIMS (see Fig. 22).

- For the brightest stars, such as $\alpha$ Ori, $\alpha$ Sco and $\gamma$ Cru, the signal-to-noise ratio of the raw, spectrally-summed data ranges from 100 to 300 per sample at our shortest integration time of $20 \mathrm{msec}$ (see Table 2). For the solar occultations the SNR is much higher: $\sim 4900$ per sample for spectrally-summed data at a wavelength of $2.3 \mu \mathrm{m}$.

- Examination of the sources of photometric noise in the occultation data permits us to estimate both the minimum and maximum detectable normal optical depths in the rings, as a function of radial resolution. For the brightest, low-inclination stars such as o Ceti, $\alpha$ Ori and $\alpha$ Sco, $\tau_{\min } \leq 0.0010$ at $1 \mathrm{~km}$ resolution, while for the brightest, high-inclination stars such as $\gamma$ Cru we find that $\tau_{\max } \simeq 4.5$ at $1 \mathrm{~km}$ resolution and $\sim 5.5$ at $10 \mathrm{~km}$ resolution (see Tables 4,5 and 10). Solar occultations provide similar values of $\tau_{\min }$ but somewhat lower values of $\tau_{\max }$, at much lower radial resolution.

- A prominent feature in almost all occultation profiles of the A and B rings, both stellar and solar, is an azimuthal asymmetry in the optical depths that is generally attributed to the presence of strong self-gravity wakes in these regions. This asymmetry is most pronounced at lower incidence angles, and is seen most clearly in chord occultations (see Figs. 5, 6 and 19). Wake parameters including estimates of the ring thickness, have been derived for regions in the A and B rings by Colwell et al. (2006), Hedman et al. (2007), Colwell et al. (2007), Nicholson and Hedman (2010) and Jerousek et al. (2016). No such asymmetry is seen in the C ring or Cassini Division, consistent with theoretical models and the lower surface mass densities in these regions.

- Immediately adjacent to many sharp gap edges in the $\mathrm{A}$ and $\mathrm{C}$ rings and in the Cassini Division we see "overshoots", or an excess in the stellar signal over the unocculted value measured well outside the rings. Examples are seen in Figs. 13 and 14. Also seen in UVIS and ground-based data from the 28 Sgr occultation, these features can 
be modeled in terms of diffraction by individual ring particles (French and Nicholson 2000; Becker et al. 2016; Harbison et al. 2019)

- Severe instrumental scattered light in the VIMS solar port images limits our sensitivity to measurements of forward-scattered sunlight, but analyses to date have found evidence for a minimum particle size of $\sim 4 \mathrm{~mm}$ in the $\mathrm{C}$ ring and $\leq 0.6 \mathrm{~mm}$ in the A ring (Harbison et al. 2013).

\section{Appendix A: Occultation geometry.}

Our procedure for the geometric reconstruction of the VIMS ring occultations follows the planetocentric method described by French et al. (1993) in their Equations (A16 A20). The basic input parameters for each occultation are a list of observation times, $t_{o}$, for each sample extracted from the SCLOCK times encoded in the raw data cubes, the date and mid-time, $t_{\text {mid }}$, of the occultation, the heliocentric position of the occulted star from the Hipparcos catalog, $\left[\alpha_{*}, \delta_{*}\right]$, and the spacecraft trajectory with respect to the center of Saturn, $\mathbf{r}_{\mathrm{sc}}(t)$, extracted from the relevant NAIF/SPICE kernel. Also needed is the direction of Saturn's pole vector, $\left[\alpha_{p}, \delta_{p}\right]$, obtained from the precessional model of Jacobson et al. (2011). The sequence of steps is as follows, where we use a variant on the compact vector notation of French et al. (1993).

- Both the occultation mid-time, $t_{\text {mid }}$, and the observation start and stop times are converted from SCLOCK to ET, using the SPICE routine 'cspice-scs2e'. Saturn's pole vector is precessed to this epoch and converted to a unit vector, $\hat{n}_{p}$.

- The components of $\hat{n}_{p}$ are used to derive a rotation matrix $\mathbf{C}$ which converts coordinates in the ICRF frame (i.e., Earth equatorial coordinates at J2000) to Saturn equatorial coordinates, where $\hat{z}$ is directed towards Saturn's north pole, $\hat{x}$ to the ascending node of Saturn's equator on the Earth equator of J2000, and $\hat{y}=\hat{z} \times \hat{x}$.

- The position and velocity of Saturn's center (body 699) relative to the Solar System Barycenter (henceforth SSB), $\mathbf{r}_{S}$ and $\mathbf{v}_{S}$, are extracted at $t_{\text {mid }}$, in the ICRF reference frame, using the SPICE routine 'cspice-spkssb'.

- The catalog position of the occulted star is corrected for proper motion from the Hipparcos epoch (1991.25) to that of the occultation mid-time, and for parallax at Saturn using $\mathbf{r}_{S}$, to yield a unit vector $\hat{\rho}$ from Saturn to the star in the reference frame of the SSB. In vector form, the parallax correction may be written

$$
\hat{\rho}_{S}=\frac{D_{*} \hat{\rho}_{H}-\mathbf{r}_{S}}{\left|D_{*} \hat{\rho}_{H}-\mathbf{r}_{S}\right|},
$$

where $\hat{\rho}_{H}$ is the heliocentric unit vector towards the star, $\pi$ is the parallax (in radians), and $D_{*}=1 A U / \pi$ is the distance to the star. 
- The stellar position is corrected for stellar aberration, to give the apparent direction to the star as seen by an observer at rest with respect to Saturn, $\hat{\rho}$ :

$$
\hat{\rho}=\frac{\hat{\rho}_{S}+\mathbf{v}_{S} / c}{\left|\hat{\rho}_{S}+\mathbf{v}_{S} / c\right|} .
$$

This vector is then converted to Saturn equatorial coordinates, using the matrix $\mathbf{C}$, and used to calculate the saturnicentric latitude, $B_{*}$ and longitude, $\lambda_{*}$, of the star, where the latter is measured from the $\hat{x}$ axis.

- The position of Cassini relative to the center of Saturn, $\mathbf{r}_{\mathrm{sc}}$, is extracted using the SPICE routine 'cspice-spkez', and tabulated at suitable intervals of time, $t_{i}$, throughout the occultation. ${ }^{17}$ These vectors are also converted to Saturn equatorial coordinates, again using the matrix C. The positions used are 'instantaneous', with no light-travel time corrections. In some cases, usually when only a provisional trajectory is available, we apply an empirical offset, $\delta t$, to the values of $t_{i}$ in order to force the radii of circular ring features to better match the values given by French et al. (1993) or French et al. (2017). We do not use such offsets once a final, reconstructed trajectory is released.

- At each instant of time, $t_{i}$, we compute the location of the intercept point of the apparent line-of-sight to the star with the ring plane $\mathbf{r}_{\mathrm{RIP}}$ as

$$
\mathbf{r}_{\mathrm{RIP}}=\mathbf{r}_{\mathrm{sc}}+\Delta \hat{\rho},
$$

where the distance, $\Delta$, from the spacecraft to the ring intercept point is determined by the requirement that $\mathbf{r}_{\mathrm{RIP}} \cdot \hat{n}_{p}=0$, or

$$
\Delta=-\frac{z_{\mathrm{sc}}}{z_{*}} .
$$

Here, $z_{*}$ is the $z$ component of the stellar unit vector $\hat{\rho}$ and the ring plane is assumed to be coincident with Saturn's equatorial plane. (Note that it is not necessary to apply light-travel time corrections to $\mathbf{r}_{\mathrm{RIP}}$ as long as the apparent direction to the star is used in this calculation (French et al. 1993).) If $\Delta$ is negative, then Cassini is on the same side of the ring plane as the star and no occultation is possible.

- The array of vectors $\mathbf{r}_{\mathrm{RIP}}\left(t_{i}\right)$ is converted to radii $r\left(t_{i}\right)$ and inertial longitudes, $\lambda\left(t_{i}\right)$ in the ring plane, where longitudes are measured in the prograde direction from the $\hat{x}$ axis. These positions are then linearly interpolated onto the original observation times, $t_{o}$.

- For use in later modeling of noncircular features, the back-dated times at which the photons pierced the ring plane, $t_{\mathrm{RIP}}=t_{o}-\Delta / c$, are also computed and stored.

\footnotetext{
${ }^{17}$ We generally use intervals of $10 \mathrm{~s}$.
} 
- The position of the ring intercept point is calculated relative to the apparent direction to the star, as defined by Hedman et al. (2007):

$$
\phi=\lambda_{\mathrm{RIP}}-\lambda_{*},
$$

for use in modeling vertical features and/or self-gravity wakes. (Note that the files delivered to the PDS list the 'Observed Ring Azimuth', defined as $\phi+180^{\circ}$ for consistency with previous occultation data sets.)

- Finally, we note that, unlike the situation with Earth-based stellar occultations, the general-relativistic deflection of the light ray by Saturn's gravity field can safely be neglected for most Cassini occultations. The maximum deflection for limb-grazing rays is $4 G M_{\text {Sat }} / c^{2} R_{\text {Sat }} \simeq 2.8 \times 10^{-8} \mathrm{rad}$, which implies a correction to the ring plane radius of $\sim 30 \cos \phi / \sin B_{*} \mathrm{~m}$ at a spacecraft distance of $1 \times 10^{6} \mathrm{~km}$.

This completes the geometric calculation for stellar occultations. The same algorithm is used for ring solar occultations, except that the apparent position of the star, $\hat{\rho}$, is replaced by the apparent direction from Saturn to the sun at the mid-time of the occultation, $\hat{\rho}_{\odot}$, calculated as

$$
\hat{\rho}_{\odot}=\frac{\mathbf{r}_{\odot}-\mathbf{r}_{S}}{\left|\mathbf{r}_{\odot}-\mathbf{r}_{S}\right|},
$$

where $\mathbf{r}_{\odot}$ is the position of the sun relative to the SSB, back-dated to allow for light travel time to Saturn. $\hat{\rho}_{\odot}$ is also corrected for stellar aberration at Saturn, using Eq. (7).

\section{Appendix B: Notes on individual occultations.}

We provide here some comments on data sets of particular interest. For numerical data the reader is referred to Tables 8,10 and 11 . Occultations are identified by the star name and Cassini orbit (or 'rev') number, as in the tables.

o Ceti (8-12): This set of four chord occultations of the famous variable star known as Mira were the first to be observed by VIMS and remain some of our highest-quality observations of the F ring and Cassini Division. This is due to a combination of the star's brightness, its very low inclination angle of $3.45^{\circ}$ and the fact that these observations occurred near apoapse on the spacecraft's orbit. As a result, the projected radial velocity of the star was $6 \mathrm{~km} \mathrm{~s}^{-1}$ or less. The track on rev 8 penetrates into the outer $\mathrm{B}$ ring, to a minimum radius of $\sim 115,000 \mathrm{~km}$, while those on revs 9 and 10 penetrate only into the middle and outer A ring, respectively. Only the F ring was crossed on rev 12. These observations led to the first estimates based on VIMS data of self-gravity wake parameters in the A ring (Hedman et al. 2007), as well as measurements of the two-dimensional structure of the star's extended envelope (Stewart et al. 2016b). The radial resolution is limited by a combination of the projected stellar diameter of $\sim 230 \mathrm{~m}$ and a radial sampling interval of up to $450 \mathrm{~m}$, but varies significantly between cuts because of the highly-foreshortened ring geometry and shallow chords. The unocculted stellar signal levels are 990-1180 DN in 80 msec. See Figs. 6 and 13. 
$\delta$ Vir (29): At $B_{*}=-2.38^{\circ}$, this is one of the lowest-inclination stars to be observed by VIMS, albeit considerably fainter than is o Ceti. Unfortunately we have only one occultation, a high-speed chord which lasted just over $1 \mathrm{hr}$. The projected radial velocity of the star was $180 \mathrm{~km} \mathrm{~s}^{-1}$, far higher than for any other VIMS occultation, leading to a radial resolution of $\sim 7 \mathrm{~km}$. Despite this limitation, the rev 29 occultation was the first to detect the diffuse $\mathrm{D}$ ring, with an average normal optical depth of $\sim 0.0005$ between radii of 73,000 and $74,000 \mathrm{~km}$.

Besides o Ceti and $\delta$ Vir, other very low-inclination stars observed by VIMS include $\gamma$ Eri, R Aql, X Oph, and $30 \mathrm{Psc}$, with $B_{*}=-7.39,5.56,5.47$, and $1.06^{\circ}$, respectively. However, none of these stars is very bright and due to the foreshortening of the rings their radial velocities are generally quite high (typically $30 \mathrm{~km} \mathrm{~s}^{-1}$ or greater). As a result, these data sets may be less useful than those of $o$ Cet and $\delta$ Vir.

$\alpha$ Sco $(13,29,55 \&$ 115): The very close chord occultation of the bright giant Antares on rev 13 was the first VIMS occultation to probe the core of the $\mathrm{B}$ ring, although the data quality is not as good as that provided by later occultations of this star due to a combination of poor pointing and excessive radiation noise. The egress portion of the track lay entirely within Saturn's shadow, as seen in Fig. 9. With Cassini's range to Saturn of only $225,000 \mathrm{~km}$, the projected radial velocity in the A ring is $\sim 10 \mathrm{~km} \mathrm{~s}^{-1}$, leading to a radial resolution of $\sim 400 \mathrm{~m}$.

Better data were acquired for $\alpha$ Sco from ingress occultations on revs 29 and 115 and a rare egress occultation on rev 55 (following a successful stellar acquisition in the Cassini Division), although none of these observations provided a complete radial profile for various reasons. (Data for the inner B and $\mathrm{C}$ rings on rev 29 were lost to data policing, while the rev 115 observation was terminated at $\sim 79,000 \mathrm{~km}$ by a Saturn occultation.) These pre-2009 occultations by $\alpha$ Sco were all observed against the unlit side of the rings. Except for rev 13 , the unocculted stellar signal levels are $670-750 \mathrm{DN}$ in $20 \mathrm{msec}$, the radial velocities are $3-5 \mathrm{~km} \mathrm{~s}^{-1}$ and the radial resolutions are $100-300 \mathrm{~m}$. With its long integration time of $80 \mathrm{msec}$, the rev 115 occultation yielded a higher stellar count rate than any other VIMS ring occultation of $\sim 2700 \mathrm{DN}$ per sample, summed over 8 channels. As a result, the 3- $\sigma$ value of $\tau_{\max }$ is a respectable 3.6 at $1 \mathrm{~km}$ resolution, despite the relatively low value of $B_{*}=-32.16^{\circ}$.

$\gamma$ Cru (71-102): This series of 17 very similar occultations of a bright southern giant almost all provided complete radial profiles covering the $\mathrm{F}, \mathrm{A}, \mathrm{B}, \mathrm{C}$ and $\mathrm{D}$ rings. The high inclination of this $\operatorname{star}\left(B_{*}=-62.35^{\circ}\right)$, plus the fact that its occultations prior to the saturnian equinox in 2009 all occurred on the dark side of the rings, resulted in generally very high-quality light curves, especially for the B ring. Although the radial velocities are all $\sim 6.5 \mathrm{~km} \mathrm{~s}^{-1}$, the radial resolution of these data sets is somewhat variable due to variations in the sampling interval, ranging from 150 to $400 \mathrm{~m}$. The unocculted stellar signal levels are 540-720 DN in 40 msec, except for revs 71, 77, 96 and 101, which suffered from poor pointing. The consistent geometry and generally high SNR of these data have made this set of occultations invaluable for studies of weak density waves in Saturn's rings (Hedman 
and Nicholson 2013, 2014, 2016, 2019), while several of these events also provide our best limits on the maximum optical depth in the central B ring. See Figs. 4 and 16.

$\alpha$ Ori (26, $46 \&$ 117): The first occultation of Betelgeuse observed by VIMS which provided complete radial coverage across the rings was that on rev 117. Earlier $\alpha$ Ori occultations on revs 26 and 46 covered only parts of the $\mathrm{A}$ and $\mathrm{F}$ rings. This is the brightest star in the sky at $2.2 \mu \mathrm{m}$. With a low inclination of $B_{*}=11.68^{\circ}$, these data are particularly well-suited to studies of the low- $\tau$ regions such as the Cassini Division, C and D rings, although for rev 117 the stellar baseline is rather unstable and the unocculted stellar flux was significantly lower than expected. At a spacecraft range of $277,000 \mathrm{~km}$, the radial velocity was a substantial $17.6 \mathrm{~km} \mathrm{~s}^{-1}$, leading to a radial resolution of $350 \mathrm{~m}$, although the projected stellar diameter for this occultation is only $50 \mathrm{~m}$, much less than for later occultations of this star (see below).

o Ceti (132, 135 \& 231): After rev 12, only a few more opportunities presented themselves to observe occultations by o Ceti, all when Cassini was on near-equatorial orbits. These were all at much closer distances than those on revs 8-12 and therefore much faster. The chord occultation on rev 132 was similar in geometry to rev 8 , but three times faster. The occultations on revs 135 and 231 provided near-radial cuts, with rev 135 yielding a full ingress profile from the $\mathrm{F}$ to $\mathrm{D}$ rings, albeit at a fairly coarse resolution of $\sim 1.0 \mathrm{~km}$ due to a high radial velocity of $16 \mathrm{~km} / \mathrm{s}$.

On rev 231, both ingress and egress occultations were observed, covering the $\mathrm{F}-\mathrm{B}$ rings on ingress and $\mathrm{C}-\mathrm{F}$ rings on egress at resolutions of $\sim 200 \mathrm{~m}$. Excellent data were acquired for the $\mathrm{C}$ ring and Cassini Division, as well as for the tenuous D ring.

$\lambda$ Vel (203): At 24:05 in duration, this was the longest stellar occultation ever observed by VIMS. It is a chord occultation that penetrates to the inner B ring, and the data were obtained using a single stellar acquisition prior to $\mathrm{F}$ ring ingress. With a projected radial velocity of only $1.4 \mathrm{~km} \mathrm{~s}^{-1}$, the resolution is limited by the stellar diameter of $180 \mathrm{~m}$. Over 1.6 million spectra were obtained at an integration time of $50 \mathrm{~ms}$, equivalent to $\sim 400$ full-size cubes.

Other particularly long occultations were those of 2 Cen on rev 237 (duration 20:05), $\mathrm{L}^{2}$ Pup on revs 199, 201 and 205 (10:30, 16:12 \& 10:35), R Lyr on revs 176 and 180 (15:23 $\&$ 13:30), $\eta$ Car on rev 250 (11:17), $\alpha$ CMa on rev 281 (10:42), VY CMa on rev 262 (10:32), W Hya on rev 236 (10:20) and $\alpha$ Sco on revs 237 and 238 (10:16 \& 10:08). Several other occultations exceeded $9 \mathrm{hrs}$ in duration. No unusual problems were encountered for most of these events, with the star generally remaining well-centered in the VIMS pixel for the duration of each observation.

$\alpha$ Sco (237-245): This series of seven moderately-distant occultations of the bright giant Antares began with two $10 \mathrm{hr}$ long B-ring chord occultations on revs 237 and 238. Subsequently the occultation track passed behind the planet, with partial ingress occultations on revs 239 and 241 and complete egress occultations on revs 241 and 243 . The sequence of observations ended with a $5 \mathrm{hr}$ chord occultation on rev 245 that penetrated all the way in 
to the D ring. During this sequence, the range to the rings decreased monotonically from $1,540,000 \mathrm{~km}$ to $442,000 \mathrm{~km}$, resulting in a radial resolution (set primarily by the projected diameter of the star) that decreased from $300 \mathrm{~m}$ to $\sim 170 \mathrm{~m}$. The radial velocity on revs 237 and 238 was an unusually low $2.4 \mathrm{~km} \mathrm{~s}^{-1}$. The rev 245 chord provided two almost complete radial profiles of the rings, except for the middle $\mathrm{C}$ ring where the ingress leg was interrupted for 24 min by a shallow occultation by Saturn, resulting in some loss of data. Data from all seven of these occultations are of good to excellent quality, even though all were observed against the sunlit rings, resulting in some background light from the rings at the 5-15 DN level. Unocculted stellar signal levels are 580-760 DN in $20 \mathrm{msec}$. The egress data on revs 241-245 include useful coverage of the outer D ring.

$\alpha$ Ori (240-277): This series consists of eleven distant occultations of the red supergiant Betelgeuse, the brightest star in the sky at $2.2 \mu \mathrm{m}$. The series began with two $4 \mathrm{hr}$ long B-ring chord occultations on revs 240 and 241, followed by shorter Cassini Division chords on revs 245 and 247, three more B ring chords on revs 253, 256 and 260, and a $5 \mathrm{hr} \mathrm{C}$ ring chord on rev 262. It ended with an unusual pair of egress occultations on revs 268 and 269, and a complete ingress occultation on rev 277. All were observed against the dark side of the rings, Unocculted stellar signal levels are $870-970$ DN per 20 msec integration, except for rev 262 where the pointing was less than ideal. The radial resolution of these data is set by a combination of sampling intervals of $100-200 \mathrm{~m}$ and the projected stellar diameter of $140-190 \mathrm{~m}$. With a fairly low inclination of $B_{*}=11.68^{\circ}$, these occultations provide especially high-quality data for the $\mathrm{F}$ ring, $\mathrm{C}$ ring and Cassini Division and have also yielded our best observations of the very low optical depth D ring. See Figs. 5, 14 and 15 for examples.

$\gamma$ Cru (255-292): A final series of seven occultations of $\gamma$ Cru provided complete radial profiles of the $\mathrm{F}-\mathrm{D}$ rings, but at closer distances than the occultations of this star on revs 71102 and at correspondingly higher radial velocities (up to $14 \mathrm{~km} / \mathrm{s}$ on revs $276-292$ ). Unlike the pre-2009 occultations, these observations were made against the sunlit rings, close to the sub-solar longitude, and so have measurable scattered light backgrounds, even at $2.92 \mu \mathrm{m}$. Despite these factors, the data quality is generally excellent and these events provide our best observations of the dense B ring in the latter part of the Cassini mission. An eighth occultation on rev 268 was planned to observe Saturn's atmosphere, but also captured the $\mathrm{C}$ and $\mathrm{D}$ rings. The radial resolution of these data sets is set by their sampling intervals of $\sim 180 \mathrm{~m}$ on revs $255-269$ and $\sim 290 \mathrm{~m}$ on revs $276-269$.

Two additional occultations of $\gamma$ Cru are also worthy of note. On rev 187 a very deep, slow chord was observed that penetrated all the way to the D ring over a period of $9.5 \mathrm{hrs}$. Two complete radial profiles were obtained with a sampling resolution of $150 \mathrm{~m}$ or less, due to the low radial velocity of $5.5-7.5 \mathrm{~km} \mathrm{~s}^{-1}$. There is some background light from the rings, amounting to $\sim 15 \mathrm{DN}$ at $2.92 \mu \mathrm{m}$. An even slower chord occultation was observed on rev 245, but in this case operational constraints limited observations to the egress half of the occultation, starting shortly before the turn-around point in the inner 
C ring at $78,800 \mathrm{~km} .{ }^{18}$ With a radial velocity of $2.1-4.3 \mathrm{~km} \mathrm{~s}^{-1}$, the sampling interval is 40-90 m. Combined with a projected stellar diameter of only $90 \mathrm{~m}$, these data provide one of the highest-resolution VIMS occultation profiles ever obtained.

$\alpha$ CMa (272-282): This short series of four distant occultations of Sirius rivals that of the $\gamma$ Cru occultation on rev 245 for resolution, but at a much lower opening angle of $13.48^{\circ}$. It consists of a complete egress occultation on rev 274 and a deep D ring chord on rev 281, book-ended by B ring chords on revs 272 and 282. All are quite slow, with radial velocities ranging from 2.0 to $6.2 \mathrm{~km} \mathrm{~s}^{-1}$, resulting in sampling resolutions of $80-250 \mathrm{~m}$ at an integration time of $40 \mathrm{~ms}$. Despite the considerable distance of $\sim 1.15 \times 10^{6} \mathrm{~km}$, the star's small angular diameter resulted in a projected size of only $30 \mathrm{~m}$ at the rings. Along with $\alpha$ Sco on rev 245, the $\alpha$ CMa occultation on rev 281 provides some of the best VIMS profiles of several tightly-wound density and bending waves in the inner $\mathrm{C}$ ring (French et al. 2019).

\section{Acknowledgements}

This work was supported by NASA, through a grant to PDN from the Cassini Project. We acknowledge the efforts of the Cassini VIMS engineering and science teams in making the acquisition of these data possible, and the PDS Rings Node for providing guidance in the processing and formatting of the occultation data. Paul Stewart provided current values for the angular diameters of the stars listed in Table 1. For many discussions of the potential for spacecraft stellar occultation observations of Saturn's rings, and of the potential utility of the VIMS instrument in carrying them out, we are indebted to Jim Elliot, Dick French, Keith Matthews, Mark Showalter, Jeffrey Cuzzi, and Bruno Sicardy. We thank our reviewers for pointing out numerous ways in which our presentation could be improved, and suggesting the addition of Table 11.

\section{Data Availability}

All of the reduced and calibrated VIMS ring stellar occultation data have been supplied, in a standardized format and at radial resolutions of $1 \mathrm{~km}$ and $10 \mathrm{~km}$, to NASA's Planetary Data System (Hedman and Nicholson 2019b). These ASCII tables consist of optical depth profiles at our standard wavelength of $2.92 \mu \mathrm{m}$, together with all necessary timing and geometric information, notes on data quality, data gaps, etc. Ingress and egress profiles are archived as separate files, so chord occultations are split across two data tables. Also available on the PDS are 'browse products' for each occultation, consisting of a set of geometric plots like those in Fig. 9. This material, along with text documents summarizing the Cassini mission, the spacecraft, the VIMS instrument and the occultation data format may be found

\footnotetext{
${ }^{18}$ Since the data do capture the turn-around point, we include this event in the list of chord occultations in Table 11.
} 
on-line at https://pds-rings.seti.org/viewmaster/volumes/COVIMS_8xxx/COVIMS_8001.

In addition to the reduced data, all of the raw VIMS occultation data files (including all 32 IR spectral channels but in a less user-friendly VIMS cube format and without any geometric information) are also available through the PDS Ring Moon Systems node (https://pds-rings.seti.org).

\section{REFERENCES}

Acton, C. H. 1996. Ancillary data services of NASA's Navigation and Ancillary Information Facility. Planetary and Space Science 44, 65-70.

Becker, T. M., Colwell, J. E., Esposito, L. W., Bratcher, A. D. 2016. Characterizing the particle size distribution of Saturn's A ring with Cassini UVIS occultation data. Icarus $279,20-35$.

Becker, T. M., Colwell, J. E., Esposito, L. W., Attree, N. O., Murray, C. D. 2018. Cassini UVIS solar occultations by Saturn's F ring and the detection of collision-produced micron-sized dust. Icarus 306, 171-199.

Brown, R. H., et al. 2004. The Cassini Visual and Infrared Mapping Spectrometer (VIMS) investigation. Space Science Reviews 115, 111-168.

Ciarniello, M., and 11 colleagues 2018. Cassini-VIMS observations of Saturn's main rings: II. A spectrophotometric study by means of Monte Carlo ray-tracing and Hapke's theory. Icarus 317, 242-265.

Clark, R. N., and 13 colleagues 2012. The surface composition of Iapetus: Mapping results from Cassini VIMS. Icarus 218, 831-860.

Clark, R. N., Brown, R. H., Lytle, D. M., Hedman, M. M. 2018. The VIMS wavelength and radiometric calibration 19. Final report. NASA Planetary Data System, Cartography and Imaging Sciences Node. https://pdsimaging.jpl.nasa.gov/data/cassini/cassini_orbiter/vims-calibration-files/clark-etal_vims-radiometric-calibration-pds-2018-v2.0.pdf

Colwell, J. E., Esposito, L. W., Sremčević, M. 2006. Self-gravity wakes in Saturn's A ring measured by stellar occultations from Cassini. Geophysical Research Letters 33, L07201.

Colwell, J. E., Esposito, L. W., Sremčević, M., Stewart, G. R., McClintock, W. E. 2007. Self-gravity wakes and radial structure of Saturn's B ring. Icarus 190, 127-144.

Colwell, J. E., Nicholson, P. D., Tiscareno, M. S., Murray, C. D., French, R. G., Marouf, E. A. 2009. The structure of Saturn's rings. In "Saturn after Cassini-Huygens". M. Dougherty \& S. Krimigis, Eds., Springer. 375-412. 
Colwell, J. E., Esposito, L. W., Jerousek, R. G., Sremčević, M., Pettis, D., Bradley, E. T. 2010. Cassini UVIS Stellar Occultation Observations of Saturn's Rings. The Astronomical Journal 140, 1569-1578.

Colwell, J., Jerousek, R., Nicholson, P., Hedman, M., Esposito, L., Harbison, R. 2014. Abundance of Small Particles in Saturn's Rings from Cassini UVIS and VIMS Stellar Occultations. EGU General Assembly Conference Abstracts 16, 2479.

Elliot, J. L. 1979. Stellar occultation studies of the solar system. Annual Review of Astronomy and Astrophysics 17, 445-475.

ESA 1997. The Hipparcos and Tycho catalogues. VizieR Online Data Catalog 1239, 0.

Esposito, L. W., Ocallaghan, M., West, R. A. 1983a. The structure of Saturn's rings Implications from the Voyager stellar occultation. Icarus 56, 439-452.

Esposito, L. W., Ocallaghan, M., Simmons, K. E., Hord, C. W., West, R. A., Lane, A. L., Pomphrey, R. B., Coffeen, D. L., Sato, M. 1983b. Voyager photopolarimeter stellar occultation of Saturn's rings. Journal of Geophysical Research 88, 8643-8649.

Esposito, L. W., and 18 colleagues 2004. The Cassini Ultraviolet Imaging Spectrograph Investigation. Space Science Reviews 115, 299-361.

Esposito, L. W., Meinke, B. K., Colwell, J. E., Nicholson, P. D., Hedman, M. M. 2008. Moonlet clumps in Saturn's F ring. Icarus 194, 278-289.

French, R. G., et al. 1993. Geometry of the Saturn system from the 3 July 1989 occultation of 28 Sgr and Voyager observations. Icarus 103, 163-214.

French, R. G., Nicholson, P. D. 2000. Particle sizes inferred inferred from stellar occultation data. Icarus $145,502-523$.

French, R. G., Nicholson, P. D., McGhee-French, C. A., Lonergan, K., Sepersky, T., Hedman, M. M., Marouf, E. A., Colwell, J. E. 2016a. Noncircular features in Saturn's rings III: The Cassini Division. Icarus 274, 131-162.

French, R. G., Nicholson, P. D., Hedman, M. M., Hahn, J. M., McGhee-French, C. A., Colwell, J. E., Marouf, E. A., Rappaport, N. J. 2016b. Deciphering the embedded wave in Saturn's Maxwell ringlet. Icarus 279, 62-77.

French, R. G., McGhee-French, C. A., Lonergan, K., Sepersky, T., Jacobson, R. A., Nicholson, P. D., Hedman, M. M., Marouf, E. A., Colwell, J. E. 2017. Noncircular features in Saturn's rings IV: Absolute radius scale and Saturn's pole direction. Icarus 290, $14-45$.

French, R. G., McGhee-French, C. A., Nicholson, P. D., Hedman, M. M. 2019. Kronoseismology III: Waves in Saturn's inner C ring. Icarus 319, 599-626. 
Gordon, M. K., Showalter, M. R., Ballard, L., Tiscareno, M., French, R. S., Olson, D. 2017. OPUS: A Comprehensive Search Tool for Remote Sensing Observations of the Outer Planets. Now with Enhanced Geometric Metadata for Cassini and New Horizons Optical Remote Sensing Instruments.. LPI Contributions 1986, 7088.

Gresh, D. L., Rosen, P. A., Tyler, G. L., Lissauer, J. J. 1986. An analysis of bending waves in Saturn's rings using Voyager radio occultation data. Icarus 68, 481-502.

Harbison, R. A., Nicholson, P. D., Hedman, M. M. 2013. The smallest particles in Saturn's A and C Rings. Icarus 226, 1225-1240.

Harbison, R. A., Nicholson, P. D., Hedman, M. M. 2019. Ring particle sizes from stellar occultations. to be submitted.

Harrington, J., Cooke, M. L., Forrest, W. J., Pipher, J. L., Dunham, E. W., Elliot, J. L. 1993. IRTF observations of the occultation of 28 SGR by Saturn. Icarus 103, 235-252.

Hedman, M. M., Nicholson, P. D. 2013. Kronoseismology: Using density waves in Saturn's $\mathrm{C}$ ring to probe the planet's interior. Astron. J. 146, 12.

Hedman, M. M., Nicholson, P. D. 2014. More Kronoseismology with Saturn's rings. Monthly Notices of the Royal Astronomical Society 444, 1369-1388.

Hedman, M. M., Nicholson, P. D. 2016. The B-ring's surface mass density from hidden density waves: Less than meets the eye? Icarus 279, 109-124.

Hedman, M. M., Nicholson, P. D. 2019. Axisymmetric density waves in Saturn's rings. Monthly Notices of the Royal Astronomical Society 485, 13-29.

Hedman, M. M., Nicholson, P. D. 2019b. Cassini Orbiter Saturn VIMS Ring Stellar Occultations 2.0. CO-SR-VIMS-4/5-OCC-V2.1, NASA Planetary Data System.

Hedman, M. M., Nicholson, P. D., Salo, H., Wallis, B. D., Buratti, B. J., Baines, K. H., Brown, R. H., Clark, R. N. 2007. Self-Gravity Wake Structures in Saturn's A Ring Revealed by Cassini VIMS. The Astronomical Journal 133, 2624-2629.

Hedman, M. M., Nicholson, P. D., Baines, K. H., Buratti, B. J., Sotin, C., Clark, R. N., Brown, R. H., French, R. G., Marouf, E. A. 2010. The architecture of the Cassini Division. Astron. J. 139, 228-251.

Hedman, M. M., Nicholson, P. D., Showalter, M. R., Brown, R. H., Buratti, B. J., Clark, R. N., Baines, K., Sotin, C. 2011. The Christiansen Effect in Saturn's narrow dusty rings and the spectral identification of clumps in the F ring. Icarus 215, 695-711.

Hedman, M. M., Nicholson, P. D., Cuzzi, J. N., Clark, R. N., Filacchione, G., Capaccioni, F., Ciarnello, M. 2013. Connection between spectra and structure in Saturn's main rings based on Cassini VIMS data. Icarus 223, 105-130.

Hedman, M. M., Nicholson, P. D., Salo, H. 2014. Exploring overstabilities in Saturn's A ring using two stellar occultations. Astron. J. 148, 15. 
Jacobson, R. A., French, R. G., Hedman, M., Colwell, J. E., Marouf, E., Rappaport, N., McGhee, C., Sepersky, T., Lonergan, K., Nicholson, P. D. 2011. The Pole Orientation, Pole Precession, and Moment of Inertia Factor of Saturn. EPSC-DPS Joint Meeting 20111070.

Jerousek, R. G., Colwell, J. E., Esposito, L. W. 2011. Morphology and variability of the Titan ringlet and Huygens ringlet edges. Icarus 216, 280-291.

Jerousek, R. G., Colwell, J. E., Esposito, L. W., Nicholson, P. D., Hedman, M. M. 2016. Small particles and self-gravity wakes in Saturn's rings from UVIS and VIMS stellar occultations. Icarus 279, 36-50.

Kliore, A. J., and 12 colleagues 2004. Cassini Radio Science. Space Science Reviews 115, $1-70$.

Marouf, E. A., Tyler, G. L., Rosen, P. A. 1986. Profiling Saturn's rings by radio occultation. Icarus $68,120-166$.

Marouf, E., French, R., Rappaport, N., McGhee, C., Thomson, F., Wong, K., Anabtawi, A. 2007. Cassini Radio Occultation Observations of the Structure and Properties of Saturn's Ring B. European Planetary Science Congress 2007757.

Marouf, E., Wong, K., French, R., Rappaport, N., McGhee, C. 2010. Cassini Radio Occultations: the Broadcast from Saturn's Rings. European Planetary Science Congress 2010803.

Matson, D. L., Lebreton, J.-P., Spilker, L. J. 2004. Cassini/Huygens in the Saturnian System. 35th COSPAR Scientific Assembly 35, 3170.

McCord, T. B., and 24 colleagues 2004. Cassini VIMS observations of the Galilean satellites including the VIMS calibration procedure. Icarus 172, 104-126.

Nicholson, P. D., Matthews, K., Goldreich, P. 1982. Radial widths, optical depths, and eccentricities of the Uranian rings. The Astronomical Journal 87, 433-447.

Nicholson, P. D., Hedman, M. M. 2010. Self-gravity wake parameters in Saturn's A and B rings. Icarus 206, 410-423.

Nicholson, P. D., Hedman, M. M. 2016. A vertical rift in Saturn's inner C ring. Icarus 279, 78-99.

Nicholson, P. D., Cooke, M. L., Pelton, E. 1990. An absolute radius scale for Saturn's rings. Astron. J. 100, 1339-1362.

Nicholson, P. D., French, R. G., Hedman, M. M., Marouf, E. A., Colwell, J. E. 2014a. Noncircular features in Saturn's rings: I. The edge of the B ring. Icarus 227, 152175 . 
Nicholson, P. D., French, R. G., Tollestrup, E., Cuzzi, J. N., Harrington, J., Matthews, K., Perković, O., Stover, R. J. 2000. Saturn's Rings I. Optical Depth Profiles from the 28 Sgr Occultation. Icarus 145, 474-501.

Nicholson, P. D., French, R. G., McGhee-French, C. A., Hedman, M. M., Marouf, E. A., Colwell, J. E., Lonergan, K., Sepersky, T. 2014b. Noncircular features in Saturn's rings: II. The $\mathrm{C}$ ring. Icarus 241, 373-396.

Pilinsky, E. B. and Lee, A. Y. 2009. Pointing-stability performance of the Cassini spacecraft. J. Spacecraft and Rockets, 46, 1007-1015.

Porco, C. C., and 34 colleagues 2005. Cassini Imaging Science: Initial Results on Saturn's Rings and Small Satellites. Science 307, 1226-1236.

Stewart, P. N., Tuthill, P. G., Nicholson, P. D., Sloan, G. C., Hedman, M. M. 2015. An Atlas of Bright Star Spectra in the Near-infrared from Cassini-VIMS. Astrophysical Journal Supplement Series 221, 30.

Stewart, P. N., Tuthill, P. G., Monnier, J. D., Ireland, M. J., Hedman, M. M., Nicholson, P. D., Lacour, S. 2016a. The weather report from IRC+10216: evolving irregular clouds envelop carbon star. Monthly Notices of the Royal Astronomical Society 455, 3102-3109.

Stewart, P. N., Tuthill, P. G., Nicholson, P. D., Hedman, M. M. 2016b. High-angularresolution stellar imaging with occultations from the Cassini spacecraft - III. Mira. Monthly Notices of the Royal Astronomical Society 457, 1410-1418.

Vahidinia, S., and 10 colleagues 2011. Saturn's F ring grains: Aggregates made of crystalline water ice. Icarus 215, 682-694. 
Table 7: Footnotes for Table 6.

\begin{tabular}{|r|l|}
\hline Number & Comment \\
\hline 1 & Unusually slow, distant occultation; covers inner A thru outer C rings only. \\
2 & Followed by a Saturn ingress occultation (separate obs.) \\
3 & Follows a Saturn egress occultation (combined obs.) \\
4 & UVIS rider. \\
5 & F and A ring data lost due to scheduling difficulties. \\
6 & F and A ring ingress lost due to trajectory shift. \\
7 & Turns around in outer C ring. \\
8 & Fast, low-inclination occultation. \\
9 & End of occultation lost to downlink. \\
10 & (Not used.) \\
11 & (Not used.) \\
12 & Inner part of ring occultation blocked by Saturn. \\
13 & All data lost due to DSN problems. \\
15 & Solar occultation accompanied a HIPHASE observation of the rings. \\
\hline
\end{tabular}


Table 8: List of all Cassini-VIMS ring stellar occultations.

\begin{tabular}{|c|c|c|c|c|c|c|c|c|}
\hline Name $(\mathrm{rev})^{a}$ & $\begin{array}{c}\text { Start time } \\
\text { (year-dayThh:mm) }\end{array}$ & $\begin{array}{r}\tau_{\mathrm{IR}}^{b} \\
(\mathrm{~ms})\end{array}$ & Mode $^{c}$ & $\begin{array}{r}F_{0}^{d} \\
(\mathrm{DN})\end{array}$ & $\begin{array}{l}\text { Duration } \\
\text { (hh:mm) }\end{array}$ & Seq. & $\begin{array}{l}\text { Qual. } \\
\text { Code }^{e}\end{array}$ & Notes $^{f}$ \\
\hline o Cet (8) & 2005-144T04:03 & 80 & $\mathrm{~S}$ & 994 & $5: 13$ & S11 & 1 & FBF chord (B4); sh \\
\hline o Cet (9) & 2005-162Т07:25 & 80 & $\mathrm{~S}$ & 996 & $3: 49$ & & $2 \mathrm{~V}$ & FAF chord; sh \\
\hline o Cet (10) & 2005-180T11:53 & 80 & $\mathrm{~S}$ & 1175 & 4:02 & S12 & $2 \mathrm{~V}$ & FAF chord; sh \\
\hline o Cet (12) & 2005-217T00:05 & 80 & $\mathrm{~S}$ & 1100 & $2: 54$ & S13 & 1 & F chord; sh \\
\hline$\alpha \operatorname{Sco}(13)$ & 2005-232T10:42 & 40 & $\mathrm{~S}$ & $\sim 775$ & $3: 55$ & & $2 \mathrm{VN}$ & FBF chord $(\mathrm{B} 2)^{17} ;$ sh \\
\hline$\alpha$ Ori $(26)$ & 2006-204T16:00 & 20 & - & 956 & $1: 00$ & S22 & 1 & $\mathrm{AB}$ rings $(\mathrm{I})^{7} ; \mathrm{sh}$ \\
\hline$\alpha \operatorname{Tau}(28)$ & 2006-252T10:00 & 40 & - & $135-150$ & $2: 15$ & S23 & $3 \mathrm{~V}$ & F-D rings $(I)^{17} ;$ sh \\
\hline$\delta \operatorname{Vir}(29)$ & 2006-268T22:00 & 40 & $\mathrm{E}$ & 125 & $1: 10$ & S24 & $3 \mathrm{~N}$ & fast FDF chord ${ }^{2}$ \\
\hline$\alpha \operatorname{Sco}(29)$ & 2006-269T06:00 & 20 & $\mathrm{~S}$ & 725 & $5: 00$ & & $1 \mathrm{D}$ & F-B rings $(\mathrm{I})^{9}$ \\
\hline R Leo (30) & 2006-285T01:30 & 20 & $\mathrm{~S}$ & 62 & $1: 30$ & & $3 \mathrm{VD}$ & fast $\mathrm{FBF}$ chord $(\mathrm{B} 3)^{17}$ \\
\hline CW Leo $(31)^{4}$ & 2006-301T01:00 & 80 & - & $\sim 200$ & $1: 30$ & S25 & $2 \mathrm{VD}$ & fast FCA chord ${ }^{10}$ \\
\hline$\alpha \operatorname{Aur}(34)$ & 2006-336T12:00 & 80 & $\mathrm{~S}$ & 410 & $2: 20$ & $\mathrm{~S} 26$ & 1 & F-D rings $(\mathrm{I}) ;$ sh \\
\hline R Hya (36) & 2007-001T16:00 & 40 & $\mathrm{~S}$ & 330 & $5: 00$ & & 1 & F-D rings $(\mathrm{I})^{2}$ \\
\hline$\alpha \operatorname{Aur}(41)$ & 2007-082T16:20 & 40 & $\mathrm{~S}$ & 191 & 4:00 & $\mathrm{S} 28$ & $1 \mathrm{~V}$ & F-D rings $(\mathrm{I})^{2} ;$ sh \\
\hline R Hya (41) & 2007-088T05:40 & 20 & $\mathrm{~S}$ & 97 & $6: 15$ & & $2 \mathrm{D}$ & F-B rings $(I)^{9}$ \\
\hline R Hya (42) & 2007-105T16:17 & 20 & $\mathrm{~S}$ & 110 & 4:00 & S29 & $1 \mathrm{~V}$ & F-A rings (I) \\
\hline$\alpha$ Ori $(46)$ & 2007-163Т01:30 & 20 & - & $\sim 700$ & $1: 00$ & S31 & $2 \mathrm{~N}$ & F-A rings (I); sh \\
\hline$o$ Cet (52) & 2007-321T06:49 & 80 & - & - & $0: 55$ & S35 & - & No useful data ${ }^{11}$ \\
\hline$\alpha \operatorname{Sco}(55)$ & 2008-003Т09:15 & 20 & $\mathrm{~S}$ & 746 & $2: 25$ & S36 & 1 & A-F rings $(\mathrm{E})$ \\
\hline$\alpha$ Aur (57) & 2008-027T22:20 & 40 & $\mathrm{~S}$ & - & $2: 55$ & S37 & - & No data ${ }^{12}$ \\
\hline R Leo (60) & 2008-063T14:45 & 40 & $\mathrm{~S}$ & 448 & $3: 00$ & S38 & 1 & FAF chord \\
\hline R Leo (61) & 2008-074T06:25 & 40 & $\mathrm{~S}$ & 420 & $2: 30$ & & $2 \mathrm{~V}$ & FAF chord \\
\hline$\alpha \operatorname{Tr} \mathrm{A}(63)$ & 2008-092Т01:35 & 80 & $\mathrm{~S}$ & 235 & $6: 20$ & S39 & 1 & FAF chord \\
\hline R Leo (63) & 2008-094T12:20 & 40 & $\mathrm{~S}$ & 360 & $3: 00$ & & $2 \mathrm{~V}$ & FBF chord (B4) \\
\hline R Cas (65) & 2008-112T00:05 & 20 & $\mathrm{~S}$ & 70 & $2: 00$ & S40 & 1 & A-D rings $(\mathrm{I})^{2,7} ; \mathrm{sh}$ \\
\hline$\alpha$ Cen (66) & 2008-120T08:00 & 60 & $\mathrm{~S}$ & 290 & $5: 35$ & S40 & 1 & $\mathrm{~F}-\mathrm{D}$ rings $(\mathrm{I})$ \\
\hline$R$ Leo (66) & 2008-123T04:40 & 40 & $\mathrm{~S}$ & - & $3: 00$ & & - & No data ${ }^{13}$ \\
\hline R Leo (68) & 2008-140T15:13 & 40 & $\mathrm{~S}$ & $\sim 50$ & $4: 31$ & & $3 \mathrm{VD}$ & FBF chord (B3) ${ }^{17}$ \\
\hline$\gamma \mathrm{Cru}(\mathrm{r0})$ & 2008-153Т04:30 & 40 & S & - & $3: 40$ & S41 & - & No useful data ${ }^{12}$ \\
\hline$\eta \operatorname{Car}(70)$ & 2008-153T11:25 & 40 & S & - & $2: 15$ & & - & No useful data ${ }^{14}$ \\
\hline
\end{tabular}

${ }^{a}$ Cassini orbit number. An 'i' for ingress and an ' $\mathrm{e}$ ' for egress is appended when both halves of an occultation were observed. Entries in italics indicate no useful data.

${ }^{b}$ Integration time for IR channel.

${ }^{c} \mathrm{~S}=$ spectrally-summed; $\mathrm{E}=$ spectrally edited.

${ }^{d}$ Average unocculted stellar counts per integration, summed over 8 spectral channels centered at $2.92 \mu \mathrm{m}$. Values in (...) are count rates at other wavelengths, typically $4.3-4.9 \mu \mathrm{m}$ for late-type stars.

${ }^{e}$ Quality codes: see Section 7 for explanation.

${ }^{f}$ Occultation geometry: $\mathrm{I}=$ ingress, $\mathrm{E}=$ egress; $\mathrm{sh}=$ track is fully or partially within Saturn's shadow; "fast" chords have $v_{\text {rad }}>20 \mathrm{~km} \mathrm{~s}^{-1}$; "slow" chords have $v_{\text {rad }}<4 \mathrm{~km} \mathrm{~s}^{-1}$. See Table 9 for numerical footnotes. 


\begin{tabular}{|c|c|c|c|c|c|c|c|c|}
\hline Name (rev) & $\begin{array}{c}\text { Start time } \\
\text { (year-dayThh:mm) }\end{array}$ & $\begin{array}{r}\tau_{\mathrm{IR}} \\
(\mathrm{ms})\end{array}$ & Mode & $\begin{array}{r}F_{0} \\
(\mathrm{DN})\end{array}$ & $\begin{array}{l}\text { Duration } \\
\text { (hh:mm) }\end{array}$ & Seq. & $\begin{array}{l}\text { Qual. } \\
\text { Code }\end{array}$ & Notes \\
\hline CW Leo $(70)^{4}$ & 2008-155T12:37 & 100 & $\mathrm{~S}$ & $\sim 350$ & $4: 53$ & S41 & $2 \mathrm{VD}$ & FBF chord (B2) \\
\hline$\gamma$ Cru (71) & 2008-160T07:50 & 40 & $\mathrm{~S}$ & $450-520$ & $3: 45$ & & $2 \mathrm{~V}$ & F-D rings $(\mathrm{I})^{2}$ \\
\hline CW Leo $(71)^{4}$ & 2008-162T15:15 & 80 & S & $\sim 180$ & $2: 45$ & & $3 \mathrm{~V}$ & $\mathrm{~F}-\mathrm{A}$ rings $(\mathrm{I})$ \\
\hline$\gamma$ Cru (72) & 2008-167T11:05 & 40 & S & 645 & $1: 55$ & & $1 \mathrm{~V}$ & F-B rings (I) \\
\hline$\gamma$ Cru (73) & 2008-174T14:15 & 40 & S & $\sim 620$ & $3: 40$ & & $2 \mathrm{~V}$ & F-D rings $(I)^{2}$ \\
\hline CW Leo $(74)^{4}$ & 2008-183Т23:45 & 40 & $\mathrm{~S}$ & 140 & $4: 15$ & S42 & $2 \mathrm{VD}$ & FBF chord (B4) \\
\hline R Leo (75) & 2008-191T03:45 & 40 & S & 268 & $3: 45$ & & $2 \mathrm{VD}$ & FBF chord (B3) \\
\hline$\gamma \mathrm{Cru}(77)$ & 2008-202T17:45 & 20 & $\mathrm{~S}$ & $80-130$ & $4: 10$ & & $3 \mathrm{~V}$ & F-D rings $(\mathrm{I})^{2,17}$ \\
\hline R Leo (77) & 2008-205T06:00 & 40 & S & 285 & $3: 35$ & & $2 \mathrm{VD}$ & FBF chord (B3) \\
\hline$\gamma \mathrm{Cru}(78)$ & 2008-209T18:50 & 20 & S & $\sim 290$ & $3: 50$ & & $2 \mathrm{VD}$ & F-D rings $(\mathrm{I})^{2}$ \\
\hline$\eta \operatorname{Car}(78)^{4}$ & 2008-209T22:40 & 40 & $\mathrm{~S}$ & 64 & $5: 20$ & $\mathrm{~S} 42$ & $2 \mathrm{~V}$ & $C D-F$ rings $(E)$ \\
\hline$\beta$ Gru (78) & 2008-210T09:00 & 20 & S & $\sim 270$ & $1: 45$ & & $3 \mathrm{~V}$ & $\mathrm{~F}-\mathrm{C}$ rings $(\mathrm{I})$ \\
\hline$\gamma \mathrm{Cru}(79)$ & 2008-216T11:00 & 40 & S & 697 & $4: 45$ & & 2 & $\mathrm{~F}-\mathrm{D}$ rings $(\mathrm{I}), 2,15$ \\
\hline RS Cnc (80) & 2008-226Т00:49 & 80 & S & 305 & $8: 16$ & $\mathrm{~S} 43$ & 1VD & FCF chord; sh \\
\hline$\gamma$ Cru (81) & 2008-231T05:25 & 40 & $\mathrm{~S}$ & $\sim 565$ & $4: 40$ & & $2 \mathrm{~V}$ & F-D rings $(I)^{2}$ \\
\hline$\gamma$ Cru (82) & 2008-238T13:50 & 40 & $\mathrm{~S}$ & 720 & $4: 40$ & $\mathrm{~S} 43$ & 1 & F-D rings $(I)^{2}$ \\
\hline RS Cnc (85) & 2008-262T20:40 & 80 & S & 311 & $9: 00$ & S44 & $1 \mathrm{D}$ & FCF chord; sh \\
\hline$\gamma$ Cru (86) & 2008-268T01:30 & 60 & $\mathrm{~S}$ & 1031 & $4: 35$ & & 1 & F-D rings $(\mathrm{I})$ \\
\hline R Leo (86) & 2008-271T09:00 & 80 & S & 650 & $3: 20$ & & $2 \mathrm{~V}$ & FAF chord \\
\hline RS Cnc (87) & 2008-277T14:15 & 80 & S & 324 & $8: 52$ & & $1 \mathrm{D}$ & FCF chord; sh \\
\hline R Leo (87) & 2008-278T18:15 & 40 & - & 304 & $2: 57$ & S44 & $2 \mathrm{~V}$ & FAF chord \\
\hline$\gamma$ Cru (89) & 2008-290T02:45 & 40 & S & 706 & $4: 40$ & & 1 & F-D rings $(I)^{2}$ \\
\hline$C W$ Leo $(89)^{4}$ & 2008-293Т07:01 & 40 & S & - & $3: 25$ & S45 & - & pointing tests only \\
\hline RS Cnc (92) & 2008-315Т00:00 & 60 & S & 224 & $4: 13$ & & $1 \mathrm{D}$ & FBB chord (B4) \\
\hline$\gamma$ Cru (93) & 2008-320T14:45 & 30 & S & $\sim 480$ & $4: 57$ & & $2 \mathrm{~V}$ & F-D rings $(\mathrm{I})^{2}$ \\
\hline$\gamma$ Cru (94) & 2008-327T23:40 & 20 & $\mathrm{~S}$ & 269 & $4: 44$ & S45 & $2 \mathrm{VD}$ & $\mathrm{F}-\mathrm{D}$ rings $(\mathrm{I})^{2}$ \\
\hline$\epsilon$ Mus (94) & 2008-328T05:59 & 60 & S & 190 & $8: 46$ & & 1 & slow FBA chord $(B 3)^{7}$ \\
\hline$\gamma \mathrm{Cru}(96)$ & 2008-343T10:08 & 20 & $\mathrm{~S}$ & $\sim 200$ & $4: 28$ & S46 & $3 \mathrm{VD}$ & F-D rings $(I)^{2}$ \\
\hline$\gamma \mathrm{Cru}(97)$ & 2008-351T09:28 & 60 & S & $\sim 840$ & $4: 27$ & & $3 \mathrm{~V}$ & F-B rings $(\mathrm{I})^{15}$ \\
\hline$\gamma$ Cru (100) & 2009-012Т08:35 & 25 & $\mathrm{~S}$ & 384 & $4: 50$ & S47 & $1 \mathrm{D}$ & F-D rings $(\mathrm{I})^{2}$ \\
\hline$\alpha \operatorname{TrA}(100)$ & 2009-013Т02:10 & 80 & $\mathrm{~S}$ & $20-25$ & $9: 30$ & S47 & $3 \mathrm{~V}$ & FCF chord $^{17}$ \\
\hline$\gamma$ Cru (101) & 2009-021T22:25 & 30 & S & $290-410$ & $4: 50$ & & $2 \mathrm{~V}$ & $\mathrm{~F}-\mathrm{D} \operatorname{rings}(\mathrm{I})^{2}$ \\
\hline$\gamma$ Cru (102) & 2009-031T11:38 & 60 & S & 990 & $4: 50$ & & $1 \mathrm{D}$ & $\mathrm{F}-\mathrm{D}$ rings $(\mathrm{I})^{2}$ \\
\hline$\alpha \operatorname{Tr} A$ (102) & 2009-032Т05:07 & 80 & S & - & $9: 15$ & & - & No useful data ${ }^{13}$ \\
\hline TX Cam (102) & 2009-034T22:14 & 40 & $\mathrm{~S}$ & $44-51$ & $5: 40$ & & $2 \mathrm{~V}$ & $\mathrm{~F}-\mathrm{C}$ rings $(\mathrm{I})$ \\
\hline$\gamma$ Cru (104) & 2009-053Т06:40 & 20 & S & 226 & $7: 35$ & S48 & $2 \mathrm{~V}$ & slow FAA chord ${ }^{7}$ \\
\hline$\beta$ Peg (104) & 2009-057T07:50 & 40 & S & $\sim 300$ & $3: 15$ & & $1 \mathrm{VD}$ & $\mathrm{F}-\mathrm{D} \operatorname{rings}(\mathrm{I})^{1}$ \\
\hline$\alpha$ Cen $(105)^{5}$ & 2009-065T17:55 & 40 & S & $\sim 190$ & $4: 50$ & & $2 \mathrm{~V}$ & F-D rings $(\mathrm{I})^{2}$ \\
\hline$\gamma$ Cru (106) & 2009-077T05:53 & 60 & S & $\sim 780$ & $7: 47$ & & $2 \mathrm{~V}$ & slow FAF chord \\
\hline R Cas (106) & 2009-081T19:59 & 20 & S & 127 & $4: 41$ & & 1 & $\mathrm{~F}-\mathrm{C} \operatorname{rings}(\mathrm{I}), \mathrm{sh}$ \\
\hline
\end{tabular}




\begin{tabular}{|c|c|c|c|c|c|c|c|c|}
\hline Name (rev) & $\begin{array}{c}\text { Start time } \\
\text { (year-dayThh:mm) }\end{array}$ & $\begin{array}{r}\tau_{\mathrm{IR}} \\
(\mathrm{ms})\end{array}$ & Mode & $\begin{array}{r}F_{0} \\
(\mathrm{DN})\end{array}$ & $\begin{array}{l}\text { Duration } \\
\text { (hh:mm) }\end{array}$ & Seq. & $\begin{array}{l}\text { Qual. } \\
\text { Code }\end{array}$ & Notes \\
\hline$\beta$ Peg (108) & 2009-095T13:10 & 40 & $\mathrm{~S}$ & 290 & $2: 59$ & S49 & $1 \mathrm{D}$ & $\mathrm{F}-\mathrm{C}$ rings $(\mathrm{I})^{21} ; \mathrm{sh}$ \\
\hline$\alpha$ Aur (110) & 2009-129T09:48 & 80 & $\mathrm{~S}$ & $250-320$ & $8: 54$ & $\mathrm{~S} 50$ & $2 \mathrm{~V}$ & FBF chord (B1) \\
\hline$\alpha$ Aur (112) & 2009-160T08:20 & 80 & $\mathrm{~S}$ & 393 & $1: 20$ & & 1 & C-D rings $(I)^{22} ;$ sh \\
\hline$\alpha \operatorname{Sco}(115)$ & 2009-208T21:20 & 80 & $\mathrm{~S}$ & 2700 & $6: 43$ & S52 & $2 \mathrm{~V}$ & $\mathrm{~F}-\mathrm{C}$ rings $(\mathrm{I})^{21}$ \\
\hline$\alpha$ Ori (117) & 2009-239T06:45 & 20 & $\mathrm{~S}$ & $400-550$ & $2: 15$ & S53 & $3 \mathrm{~V}$ & F-D rings $(I)^{1,17} ;$ sh \\
\hline$o$ Cet (132) & 2010-154T05:55 & 80 & - & 400 & $1: 55$ & $\mathrm{~S} 60$ & $3 \mathrm{VBDN}$ & fast FBF chord (B4) \\
\hline$o$ Cet (135) & 2010-205T19:15 & 60 & $\mathrm{~S}$ & $\sim 330$ & $2: 27$ & S61 & 2VBD & F-D rings $(\mathrm{I})^{1}$ \\
\hline$\alpha \mathrm{CMa}(168)^{5}$ & 2012-180T22:45 & 20 & $\mathrm{~S}$ & 39 & $1: 30$ & S74 & $2 \mathrm{~N}$ & $\mathrm{C}-\mathrm{D}$ rings $(\mathrm{I})^{22} ; \mathrm{sh}$ \\
\hline$\alpha$ CMa (169) & 2012-204T20:20 & 20 & $\mathrm{~S}$ & 38 & $1: 56$ & & $2 \mathrm{VN}$ & C-D rings $(\mathrm{I})^{22} ; \mathrm{sh}$ \\
\hline$\beta$ Peg (170) & 2012-224T14:25 & 40 & $\mathrm{~S}$ & 280 & $6: 25$ & & 1 & $\mathrm{C}-\mathrm{A}$ rings $(\mathrm{E})^{15} ; \mathrm{sh}$ \\
\hline$\beta$ Peg (172) & 2012-266T17:02 & 40 & $\mathrm{~S}$ & 278 & $6: 05$ & S75 & $1 \mathrm{D}$ & F-D rings $(\mathrm{I})^{2}$ \\
\hline$\lambda$ Vel (173) & 2012-292Т09:31 & 60 & $\mathrm{~S}$ & 250 & $3: 22$ & & $1 \mathrm{D}$ & $\mathrm{F}-\mathrm{C}$ rings $(\mathrm{I})^{21}$ \\
\hline$\alpha \operatorname{Cet}(174)$ & 2012-315T09:15 & 80 & - & 245 & $3: 16$ & S76 & $2 \mathrm{VD}$ & fast $\mathrm{FCB}$ chord $^{8}$ \\
\hline$\alpha \operatorname{Lyr}(175)^{5}$ & 2012-324T07:12 & 80 & - & 64 & $8: 28$ & & $2 \mathrm{~V}$ & F-D rings $(\mathrm{I})$ \\
\hline R Lyr (176) & 2012-339T14:15 & 40 & $\mathrm{~S}$ & 230 & $15: 23$ & & $1 \mathrm{D}$ & slow FBA chord (B3) ${ }^{10}$ \\
\hline W Hya (179) & 2013-019T18:52 & 20 & $\mathrm{~S}$ & 250 & 4:04 & S77 & $1 \mathrm{D}$ & F-D rings $(\mathrm{I})$ \\
\hline R Lyr (180) & 2013-026T17:52? & 40 & $\mathrm{~S}$ & 230 & $13: 30$ & & $1 \mathrm{D}$ & slow FBA chord $(\mathrm{B} 3)^{10}$ \\
\hline R Cas (180) & 2013-030T09:32? & 40 & $\mathrm{~S}$ & 110 & $4: 16$ & & 2 & $\mathrm{~F}$ ring chord \\
\hline W Hya (180) & 2013-033Т02:09 & 20 & $\mathrm{~S}$ & 235 & $3: 20$ & & $2 \mathrm{VD}$ & $\mathrm{F}-\mathrm{D}$ rings $(\mathrm{I})$ \\
\hline W Hya (181) & 2013-046T09:22 & 20 & $\mathrm{~S}$ & 265 & 4:06 & & $1 \mathrm{D}$ & F-D rings $(\mathrm{I})$ \\
\hline$\mu$ Cep (185) & 2013-090T13:41 & 40 & $\mathrm{~S}$ & 200 & $7: 00$ & S78 & $2 \mathrm{VD}$ & D-F rings $(E) ;$ sh \\
\hline R Cas (185) & 2013-091T01:41 & 40 & $\mathrm{~S}$ & 75 & $6: 19$ & & $2 \mathrm{VD}$ & F-D rings $(\mathrm{I})$ \\
\hline R Hya (185) & 2013-094T07:20 & 40 & S & 250 & $7: 00$ & & $2 \mathrm{VD}$ & FBA chord (B1) \\
\hline R Dor $(186)^{4}$ & 2013-102T15:05 & 20 & $\mathrm{~S}$ & 600 & $2: 55$ & & 1 & FBA chord $(\mathrm{B} 5)^{10}$ \\
\hline W Hya (186) & 2013-103Т19:36 & 20 & S & 245 & $3: 30$ & & $1 \mathrm{D}$ & $\mathrm{C}-\mathrm{F}$ rings $(\mathrm{E})$ \\
\hline$\gamma$ Cru (187) & 2013-112T13:15 & 20 & $\mathrm{~S}$ & 320 & $9: 30$ & S78 & $2 \mathrm{VD}$ & FDF chord \\
\hline R Dor (188) & 2013-121T18:30 & 20 & $\mathrm{~S}$ & 520 & $4: 46$ & & $2 \mathrm{~V}$ & FBF chord (B4) \\
\hline W Hya (189) & 2013-132T12:41 & 20 & S & 280 & $3: 25$ & & $1 \mathrm{D}$ & $\mathrm{C}-\mathrm{F}$ rings $(\mathrm{E})$ \\
\hline$\mu$ Cep (191) & 2013-148T19:30 & 40 & $\mathrm{~S}$ & 220 & $6: 00$ & & $2 \mathrm{VD}$ & F-D rings $(\mathrm{I})^{2}$ \\
\hline R Cas (191) & 2013-149T17:24 & 40 & S & 100 & $5: 09$ & & $2 \mathrm{VD}$ & F-D rings $(\mathrm{I})^{2}$ \\
\hline R Car (191) & 2013-152T17:30 & 40 & $\mathrm{~S}$ & 85 & $3: 50$ & S78 & $2 \mathrm{VD}$ & F-D rings $(\mathrm{I})$ \\
\hline R Cas (192) & 2013-161T16:24 & 40 & $\mathrm{~S}$ & $\sim 55$ & $2: 36$ & S79 & $3 \mathrm{~V}$ & F-B rings $(\mathrm{I})^{17}$ \\
\hline$\mu$ Cep (193) & 2013-172T17:35 & 40 & S & 240 & $5: 55$ & & $1 \mathrm{D}$ & F-D rings (I) \\
\hline$\mu$ Cep (194) & 2013-184T16:00 & 20 & S & 105 & $3: 42$ & & 1 & F-B rings (I) \\
\hline R Cas (194) & 2013-185T23:22 & 40 & $\mathrm{~S}$ & 105 & $5: 43$ & & $1 \mathrm{~V}$ & $\mathrm{D}-\mathrm{F}$ rings $(\mathrm{E}), \mathrm{sh}$ \\
\hline$\eta \operatorname{Car}(194)^{4}$ & 2013-188T18:06 & 80 & $\mathrm{~S}$ & $105-125$ & $3: 54$ & S79 & $2 \mathrm{VD}$ & F-D rings $(\mathrm{I})$ \\
\hline 2 Cen (194) & 2013-189T13:06 & 80 & S & 345 & $8: 54$ & & $2 \mathrm{VD}$ & FDA chord ${ }^{6}$ \\
\hline$\mu$ Cep (195) & 2013-196T15:00 & 40 & $\mathrm{~S}$ & 225 & $4: 45$ & & $2 \mathrm{~V}$ & F-B rings $(\mathrm{I})$ \\
\hline W Hya (196) & 2013-229T17:00 & 20 & $\mathrm{~S}$ & 340 & $6: 41$ & $\mathrm{~S} 80$ & 2 & FBA chord $(\mathrm{B} 1)^{15}$ \\
\hline$\beta$ And (196) & 2013-241T00:00 & 100 & $\mathrm{~S}$ & 460 & $7: 45$ & & $1 \mathrm{VB}$ & slow FAF chord ${ }^{96}$ \\
\hline
\end{tabular}




\begin{tabular}{|c|c|c|c|c|c|c|c|c|}
\hline Name (rev) & $\begin{array}{c}\text { Start time } \\
\text { (year-dayThh:mm) }\end{array}$ & $\begin{array}{r}\tau_{\mathrm{IR}} \\
(\mathrm{ms})\end{array}$ & Mode & $\begin{array}{r}F_{0} \\
(\mathrm{DN})\end{array}$ & $\begin{array}{l}\text { Duration } \\
\text { (hh:mm) }\end{array}$ & Seq. & $\begin{array}{l}\text { Qual. } \\
\text { Code }\end{array}$ & Notes \\
\hline W Hya (197) & 2013-253T15:22 & 20 & $\mathrm{~S}$ & 310 & $5: 45$ & S 80 & $1 \mathrm{D}$ & FBA chord $(\mathrm{B} 2)^{10}$ \\
\hline $\mathrm{L}^{2}$ Pup (198) & 2013-281T11:40 & 80 & S & 210 & $7: 10$ & & $2 \mathrm{~V}$ & slow FAA chord ${ }^{18}$ \\
\hline R Lyr (198) & 2013-289T07:00 & 40 & $\mathrm{~S}$ & 235 & 5:05 & & 1 & F-D rings $(I)$ \\
\hline $\mathrm{L}^{2} \operatorname{Pup}(199)$ & 2013-327Т06:30 & 40 & S & 100 & $10: 30$ & S 81 & $2 \mathrm{~V}$ & $\mathrm{C}-\mathrm{F}$ rings $(\mathrm{E})$ \\
\hline R Lyr (199i) & 2013-336 T23:00 & 40 & S & 215 & $6: 00$ & & $1 \mathrm{~V}$ & F-D rings $(\mathrm{I})$ \\
\hline R Lyr (199e) & 2013-337T06:00 & 40 & S & 215 & $4: 05$ & S81 & 1 & $\mathrm{~B}-\mathrm{F}$ rings $(\mathrm{E})$ \\
\hline R Lyr (200) & 2014-003Т16:24 & 40 & $\mathrm{~S}$ & 220 & $4: 12$ & S82 & 1 & F-D rings $(\mathrm{I})$ \\
\hline $\mathrm{L}^{2} \operatorname{Pup}(201 \mathrm{a})$ & 2014-022Т09:48 & 40 & S & 130 & $16: 12$ & & $2 \mathrm{VD}$ & slow FBF chord (B4) \\
\hline$\gamma$ Eri $(201)$ & 2014-041T18:12 & 40 & $\mathrm{~S}$ & 85 & $3: 20$ & & $2 \mathrm{~V}$ & FCDF chord $^{97}$ \\
\hline$L^{2} \operatorname{Pup}(201 b)$ & 2014-050T22:10 & 40 & S & 115 & $8: 44$ & & $2 \mathrm{VD}$ & F-D rings (I); sh \\
\hline$\alpha \operatorname{Lyr}(202 \mathrm{i})^{5}$ & 2014-067T02:30 & 80 & $\mathrm{~S}$ & 30 & $3: 35$ & S82 & 2 & F-D rings $(\mathrm{I})$ \\
\hline R Lyr (202i) & 2014-067T06:05 & 40 & S & 185 & $2: 58$ & & $2 \mathrm{~V}$ & $\mathrm{~F}-\mathrm{C}$ rings $(\mathrm{I})^{21}$ \\
\hline$\alpha \operatorname{Lyr}(202 \mathrm{e})^{5}$ & 2014-067T09:03 & 80 & $\mathrm{~S}$ & 29 & $2: 21$ & & 2 & $\mathrm{C}-\mathrm{F}$ rings $(\mathrm{E}) ; \mathrm{sh}$ \\
\hline R Lyr (202e) & 2014-067T11:24 & 40 & $\mathrm{~S}$ & 185 & $3: 54$ & & $2 \mathrm{~V}$ & $\mathrm{D}-\mathrm{F}$ rings $(\mathrm{E}) ;$ sh \\
\hline$\lambda$ Vel (203) & 2014-084T14:13 & 50 & $\mathrm{~S}$ & 170 & $24: 05$ & S83 & $2 \mathrm{D}$ & slow FBF chord (B2) \\
\hline$L^{2} \operatorname{Pup}(205 i)$ & 2014-174T20:35 & 40 & $\mathrm{~S}$ & 130 & $7: 43$ & S84 & $2 \mathrm{~V}$ & $\mathrm{~F}-\mathrm{C}$ rings $(\mathrm{I})^{21}$ \\
\hline $\mathrm{L}^{2} \operatorname{Pup}(205 \mathrm{e})$ & 2014-175T08:18 & 40 & $\mathrm{~S}$ & 130 & $10: 35$ & & 2 & $\mathrm{D}-\mathrm{F}$ rings $(\mathrm{E})$ \\
\hline$\alpha \operatorname{Lyr}(206)^{5}$ & 2014-197T21:01 & 60 & $\mathrm{~S}$ & 43 & $4: 17$ & & 2 & F-D rings (I) \\
\hline R Lyr (206) & 2014-198T01:18 & 20 & $\mathrm{~S}$ & 100 & 1:42 & & 1 & F-B rings (I) \\
\hline $\mathrm{L}^{2} \operatorname{Pup}(206)$ & 2014-206 T16:40 & 40 & $\mathrm{~S}$ & 80 & $6: 47$ & & $2 \mathrm{~V}$ & $\mathrm{D}-\mathrm{F}$ rings $(\mathrm{E})$ \\
\hline R Lyr (208) & 2014-262Т08:43 & 40 & $\mathrm{~S}$ & $\sim 200$ & $4: 32$ & S85 & $2 \mathrm{VB}$ & $\mathrm{D}-\mathrm{F}$ rings $(\mathrm{E})^{20}$ \\
\hline$\alpha \operatorname{Lyr}(209)^{5}$ & 2014-294T12:55 & 80 & - & 65 & $1: 20$ & S 86 & 1 & $\mathrm{D}-\mathrm{C}$ rings $(\mathrm{E})^{3} ; \operatorname{sh} ?$ \\
\hline$\alpha \operatorname{Her}(211)$ & 2015-009T06:21 & 20 & S & 360 & $2: 46$ & S87 & $1 \mathrm{~V}$ & FAF chord \\
\hline$\alpha \operatorname{Her}(212)$ & 2015-041T10:30 & 20 & $\mathrm{~S}$ & 375 & $1: 10$ & S 88 & 1 & F-B rings $(\mathrm{I})^{23}$ \\
\hline X Oph (213) & 2015-073Т11:49 & 20 & S & 35 & $2: 51$ & & $1 \mathrm{~V}$ & F-D rings $(\mathrm{I})$ \\
\hline 30 Psc (222) & 2015-273Т15:25 & 20 & S & 17 & $2: 44$ & S91 & $3 \mathrm{DBN}$ & FAF chord \\
\hline 30 Psc (225) & 2015-315Т08:36 & 20 & S & - & 2:50 & & - & No data ${ }^{12}$ \\
\hline$o$ Cet (231i) & 2016-030T07:06 & 20 & $\mathrm{~S}$ & 128 & $2: 26$ & S92 & 1 & F-B rings $(\mathrm{I})^{23}$ \\
\hline$o$ Cet $(231 \mathrm{e})$ & 2016-030T11:41 & 20 & $\mathrm{~S}$ & 128 & $2: 42$ & & 1 & $\mathrm{C}-\mathrm{F}$ rings $(\mathrm{E})$ \\
\hline R Aql (233) & 2016-069T16:00 & 40 & S & 52 & $2: 15$ & S93 & $3 \mathrm{~V}$ & fast $\mathrm{FCF}$ chord ${ }^{15}$ \\
\hline$\epsilon \operatorname{Peg}(233)$ & 2016-069T18:15 & 40 & $\mathrm{~S}$ & 80 & $2: 14$ & S93 & $2 \mathrm{~B}$ & F-D rings $(I)^{15}$ \\
\hline W Hya (236) & 2016-148T01:40 & 60 & $\mathrm{~S}$ & $\sim 750$ & $10: 20$ & S94 & $2 \mathrm{VB}$ & $\mathrm{F}-\mathrm{C}$ rings $(\mathrm{I})^{21} ; \mathrm{sh}$ \\
\hline 2 Cen (237) & 2016-173Т09:25 & 60 & S & $220-260$ & 20:05 & & $2 \mathrm{VBD}$ & slow FBA chord $(\mathrm{B} 2)^{10}$ \\
\hline$\alpha \operatorname{Sco}(237)$ & 2016-177T11:49 & 60 & $\mathrm{~S}$ & 2290 & $10: 16$ & & 1B & slow FBF chord (B4) \\
\hline$\beta$ Peg (237) & 2016-180T20:24 & 40 & $\mathrm{~S}$ & 270 & $5: 30$ & S95 & 1 & FBF chord (B5) \\
\hline$\alpha \operatorname{Sco}(238)$ & 2016-201T10:21 & 60 & $\mathrm{~S}$ & 1736 & 10:08 & S95 & $2 \mathrm{VB}$ & slow FBA chord (B4) $)^{10,20}$ \\
\hline$\alpha \operatorname{Sco}(239)$ & 2016-218T15:44 & 20 & S & 580 & $2: 52$ & & $2 \mathrm{~V}$ & F-B rings $(\mathrm{I})^{23} ; \mathrm{sh}$ \\
\hline R Cas (239) & 2016-220T20:53 & 40 & $\mathrm{~S}$ & 100 & $6: 22$ & & $1 \mathrm{D}$ & FBF chord (B2) \\
\hline$\rho$ Per $(239)$ & 2016-221T13:20 & 40 & $\mathrm{~S}$ & 95 & $2: 30$ & & 1 & C-F rings (E); sh \\
\hline$\alpha$ Ori (240) & 2016-234T12:35 & 20 & $\mathrm{~S}$ & 950 & 4:00 & & $1 \mathrm{D}$ & FBF chord (B3) \\
\hline
\end{tabular}




\begin{tabular}{|c|c|c|c|c|c|c|c|c|}
\hline Name (rev) & $\begin{array}{c}\text { Start time } \\
\text { (year-dayThh:mm) }\end{array}$ & $\begin{array}{r}\tau_{\mathrm{IR}} \\
(\mathrm{ms})\end{array}$ & Mode & $\begin{array}{r}F_{0} \\
(\mathrm{DN})\end{array}$ & $\begin{array}{l}\text { Duration } \\
\text { (hh:mm) }\end{array}$ & Seq. & $\begin{array}{l}\text { Qual. } \\
\text { Code }\end{array}$ & Notes \\
\hline$\alpha \operatorname{Sco}(241 \mathrm{i})$ & 2016-243T11:00 & 20 & $\mathrm{~S}$ & 660 & $2: 44$ & S95 & 1 & F-B rings $(\mathrm{I})^{23} ;$ sh \\
\hline$\alpha \operatorname{Sco}(241 \mathrm{e})$ & 2016-243Т13:44 & 20 & S & 680 & $4: 28$ & & $1 \mathrm{D}$ & $\mathrm{D}-\mathrm{F}$ rings $(\mathrm{E})$ \\
\hline X Oph (241) & 2016-244T04:55 & 20 & $\mathrm{~S}$ & 33 & $1: 25$ & & 1 & fast FCF chord \\
\hline$\alpha$ Ori $(241)$ & 2016-246T11:32 & 20 & $\mathrm{~S}$ & $\sim 880$ & $3: 53$ & & $2 \mathrm{VD}$ & FBF chord (B3) \\
\hline$\alpha \operatorname{Sco}(243)$ & 2016-267T11:44 & 20 & S & 695 & 5:06 & S96 & $1 \mathrm{D}$ & D-F rings $(E)$ \\
\hline X Oph (243) & 2016-268T02:56 & 20 & S & $\sim 24$ & $2: 02$ & S96 & $3 \mathrm{~V}$ & fast FCF chord \\
\hline R Cas (243) & 2016-268T14:43 & 40 & $\mathrm{~S}$ & 68 & $3: 32$ & & $2 \mathrm{~V}$ & F-D rings $(\mathrm{I})$ \\
\hline$\lambda$ Vel (245) & 2016-284T15:45 & 90 & S & 44 & $8: 55$ & & $3 \mathrm{~V}$ & FAF chord \\
\hline$\gamma$ Cru (245) & 2016-286T07:27 & 20 & $\mathrm{~S}$ & 290 & $6: 35$ & & $1 \mathrm{D}$ & $\mathrm{C}-\mathrm{F}$ rings $(\mathrm{E})^{90}$ \\
\hline$\alpha \operatorname{Sco}(245)$ & 2016-287T01:10 & 20 & $\mathrm{~S}$ & 700 & $5: 29$ & & $1 \mathrm{D}$ & FDF chord $^{6}$ \\
\hline$\alpha$ Ori $(245)$ & 2016-289T11:27 & 20 & $\mathrm{~S}$ & 920 & $3: 39$ & S96 & 1 & FCDF chord $^{98}$ \\
\hline$\lambda$ Vel (246) & 2016-294T05:21 & 80 & $\mathrm{~S}$ & 275 & $5: 15$ & & $2 \mathrm{~V}$ & FA partial chord \\
\hline$\alpha$ Cen (247) & 2016-305T17:40 & 80 & - & $200-250$ & $4: 15$ & & 3 & F chord (graze) ${ }^{19}$ \\
\hline$\alpha$ Ori (247) & 2016-308T14:25 & 20 & $\mathrm{~S}$ & $\sim 870$ & $3: 45$ & & $1 \mathrm{~V}$ & FCDF chord $^{99}$ \\
\hline$\eta \operatorname{Car}(250)^{4}$ & 2016-331T08:43 & 80 & S & $\sim 55$ & $11: 17$ & S97 & $2 \mathrm{VD}$ & FCF chord $^{17}$ \\
\hline$\alpha$ Ori $(253)$ & 2016-355T08:19 & 20 & S & 930 & $4: 16$ & S97 & 1 & FBF chord (B5) \\
\hline$\gamma$ Cru (255) & 2017-001T18:11 & 20 & $\mathrm{~S}$ & $\sim 255$ & $3: 24$ & & $2 \mathrm{~V}$ & F-D rings $(\mathrm{I})^{2} ;$ sh \\
\hline VY CMa (256) & 2017-007T03:10 & 80 & $\mathrm{~S}$ & $\sim 85$ & $8: 35$ & & $2 \mathrm{VN}$ & slow FBF chord (B4) \\
\hline$\alpha$ Ori $(256)$ & 2017-010T20:00 & 20 & $\mathrm{~S}$ & $\sim 910$ & $4: 36$ & & $1 \mathrm{VD}$ & FBF chord (B3) \\
\hline$\alpha$ Ori $(260)$ & 2017-039T11:56 & 20 & $\mathrm{~S}$ & 900 & $5: 08$ & S98 & $1 \mathrm{VD}$ & FBF chord (B1) \\
\hline VY CMa (262) & 2017-050T03:09 & 80 & $\mathrm{~S}$ & 160 & $10: 32$ & S98 & $2 \mathrm{VD}$ & slow FBF chord (B1) \\
\hline$\alpha$ Ori $(262)$ & 2017-053Т20:10 & 20 & S & $600-700$ & $5: 24$ & & $3 \mathrm{~V}$ & FCF chord \\
\hline$\gamma$ Cru (264) & 2017-066T07:04 & 20 & S & 270 & $2: 46$ & & $1 \mathrm{~V}$ & F-D rings $(I)^{1} ;$ sh \\
\hline$\lambda$ Vel (265) & 2017-072Т20:56 & 80 & $\mathrm{~S}$ & $\sim 20$ & $4: 24$ & & $3 \mathrm{~V}$ & $\mathrm{~F}-\mathrm{C}$ rings $(\mathrm{I})^{2,17} ; \mathrm{sh}$ \\
\hline$\lambda$ Vel (268) & 2017-094T08:23 & 80 & $\mathrm{~S}$ & $\sim 200$ & $4: 46$ & & $2 \mathrm{~V}$ & F-D rings $(\mathrm{I})^{2} ; \mathrm{sh}$ \\
\hline$\gamma$ Cru (268) & 2017-095T00:39 & 20 & S & 320 & $2: 10$ & S98 & 1 & $\mathrm{C}-\mathrm{D}$ rings $(\mathrm{I})^{22} ; \mathrm{sh}$ \\
\hline$\alpha$ Ori $(268)$ & 2017-096T22:00 & 20 & S & 900 & $3: 36$ & & $1 \mathrm{D}$ & $\mathrm{D}-\mathrm{F}$ rings $(\mathrm{E})^{1}$ \\
\hline VY CMa (269) & 2017-100T06:20 & 80 & $\mathrm{~S}$ & $\sim 220$ & $6: 12$ & & $1 \mathrm{VD}$ & F-D rings $(\mathrm{I})$ \\
\hline$\eta$ Car $(269)^{4}$ & 2017-101T23:00 & 80 & $\mathrm{~S}$ & 117 & 4:03 & & $2 \mathrm{VD}$ & $\mathrm{F}-\mathrm{C}$ rings $(\mathrm{I})$ \\
\hline$\gamma$ Cru (269) & 2017-102Т03:03 & 20 & $\mathrm{~S}$ & 320 & 3:06 & & 1 & F-D rings $(\mathrm{I})^{2} ;$ sh \\
\hline$\alpha$ Ori (269) & 2017-104T02:05 & 20 & $\mathrm{~S}$ & $\sim 900$ & $3: 15$ & S98 & $1 \mathrm{VD}$ & D-F rings $(\mathrm{E})$ \\
\hline$\alpha \mathrm{CMa}(272)$ & 2017-120T17:36 & 40 & $\mathrm{~S}$ & $50-90$ & 8:04 & S99 & $3 \mathrm{VD}$ & slow FBF chord $(\mathrm{B} 2)^{17}$ \\
\hline$\alpha \mathrm{CMa}(274)$ & 2017-133T18:36 & 40 & $\mathrm{~S}$ & 125 & $5: 27$ & & $1 \mathrm{VD}$ & D-F egress \\
\hline$\gamma$ Cru (276) & 2017-148T09:35 & 20 & S & 215 & $2: 42$ & S100 & $2 \mathrm{~V}$ & F-D rings $(\mathrm{I})^{2} ;$ sh \\
\hline$\alpha$ Ori $(277)$ & 2017-155T21:30 & 20 & $\mathrm{~S}$ & $\sim 970$ & $2: 35$ & & 1 & F-D rings $(\mathrm{I})^{1}$ \\
\hline$\alpha \mathrm{CMa}(281)$ & 2017-178T16:54 & 40 & S & $115-130$ & $10: 42$ & S100 & $2 \mathrm{VBD}$ & slow FDF chord \\
\hline$\alpha \mathrm{CMa}(282)$ & 2017-185T04:36 & 40 & S & $\sim 120$ & $9: 36$ & & $2 \mathrm{VBD}$ & slow FBF chord (B1) \\
\hline$\gamma$ Cru (282) & 2017-187T04:58 & 20 & S & $\sim 260$ & $2: 30$ & & $2 \mathrm{~V}$ & F-D rings $(\mathrm{I})^{2} ; \mathrm{sh}$ \\
\hline$\gamma$ Cru $(291)$ & 2017-245T09:06 & 20 & S & $\sim 260$ & $1: 39$ & S101 & $2 \mathrm{~V}$ & F-D rings $(\mathrm{I})^{1} ;$ sh \\
\hline$\gamma$ Cru $(292)$ & 2017-251T20:10 & 20 & $\mathrm{~S}$ & 315 & $1: 38$ & & 1 & F-D rings $(\mathrm{I})^{1} ;$ sh \\
\hline
\end{tabular}


Table 9: Footnotes for Table 8.

\begin{tabular}{|r|l|}
\hline Number & Comment ${ }^{a}$ \\
\hline 1 & Followed by a Saturn ingress occultation (separate observation \\
2 & and/or separate design). \\
& Followed by a Saturn ingress occultation (combined observation \\
3 & and design). \\
4 & UVIS rider on a Saturn egress occultation; inner C ring only. (209) \\
5 & UVIS rider, targeted using UVIS-HSP boresight. \\
6 & Grazing Saturn chord occultation blocks part of C ring on egress \\
7 & (194) or ingress (245). \\
8 & F ring missed due to trajectory shift. $(26,94,104)$ \\
9 & A ring \& Cassini Div. egress missed due to data policing. (174) \\
10 & Truncated on egress within A ring. \\
11 & All ring data lost due to data policing. (52) \\
12 & Star acquisition failed. (57, 70, 225) \\
13 & All data lost due to DSN problems. (66, 102) \\
14 & Stellar signal lost during occultation; combined with $\gamma$ Cru. (70) \\
15 & Partial loss of data due to DSN problems. \\
16 & (Not used.) \\
17 & Low signal due to bad star acquisition. \\
18 & F ring egress observed at $\tau_{\text {IR }}=40$ ms. (198) \\
19 & A ring appulse observed at $\tau_{\text {IR }}=40$ ms between F ring \\
20 & occultations. (247) \\
21 & Background signal unsummed. (208, 238) \\
22 & Terminated by Saturn ingress in C ring. \\
23 & Short ring occultation preceding Saturn ingress; inner C ring only. \\
90 & Terminated by Saturn ingress in B ring. (212, 231, 239, 241) \\
96 & Half-chord occ; begins in inner C ring. (245) \\
97 & Turns around in Encke Gap. (196) \\
98 & Turns around just inside Kuiper Gap. (201) \\
99 & Turns around just inside Laplace Gap. (245) \\
\hline
\end{tabular}

${ }^{a}$ Rev numbers listed in parentheses when there are fewer than 5 instances. 
Table 10: Geometric \& photometric data for Cassini-VIMS ring occultations.

\begin{tabular}{|c|c|c|c|c|c|c|c|c|}
\hline Name (rev) & $\begin{array}{r}B_{*} \\
(\mathrm{deg}) \\
\end{array}$ & $\begin{array}{r}D^{a} \\
(\mathrm{Mm})\end{array}$ & $\begin{array}{l}D \theta_{*} \\
(\mathrm{~m}) \\
\end{array}$ & $\begin{array}{r}\text { F.Z. }{ }^{b} \\
(\mathrm{~m})\end{array}$ & $\begin{array}{r}F_{0}^{c} \\
(\mathrm{DN}) \\
\end{array}$ & $\begin{array}{c}\left|v_{\mathrm{rad}}\right|^{d} \\
(\mathrm{~km} / \mathrm{s})\end{array}$ & $\tau_{\min }^{e}$ & $\tau_{\max }^{f}$ \\
\hline omiCet (8) & 3.45 & 1640. & 231. & 99. & 994. & 5.71 & 0.0001 & 0.38 \\
\hline omiCet (9) & 3.45 & 1618. & 228 . & 99. & 996. & 7.00 & 0.0001 & 0.37 \\
\hline omiCet (10) & 3.45 & 1613. & 227. & 98. & 1175. & 7.00 & 0.0001 & 0.38 \\
\hline omiCet (12) & 3.45 & 1579. & 222 . & 97. & 1100. & 7.00 & 0.0001 & 0.38 \\
\hline alpSco (13) & -32.16 & 225 . & 44. & 37. & 775. & 10.28 & 0.0014 & 3.25 \\
\hline alpOri (26) & 11.68 & 316. & 57. & 44. & 956. & 10.66 & 0.0003 & 1.35 \\
\hline alpTau (28) & 22.17 & 345 . & 36. & 46. & 137. & 12.80 & 0.0055 & 1.61 \\
\hline delVir (29) & -2.38 & 265. & 13. & 40. & 125. & 181.58 & 0.0025 & 0.12 \\
\hline alpSco (29) & -32.16 & 516. & 100. & 56. & 725. & 5.25 & 0.0007 & 3.58 \\
\hline R Leo (30) & 9.55 & 349. & 47. & 46. & 62. & 26.72 & 0.0053 & 0.57 \\
\hline CW Leo (31) & 11.38 & 286. & 72. & 41. & 200. & 40.27 & 0.0050 & 0.73 \\
\hline alpAur (34) & 50.88 & 401. & 10. & 49. & 410. & 11.36 & 0.0053 & 3.93 \\
\hline R Hya (36) & -29.40 & 914. & 111. & 74. & 330. & 8.81 & 0.0025 & 2.61 \\
\hline alpAur (41) & 50.88 & 601. & 15. & 60. & 191. & 7.72 & 0.0063 & 3.76 \\
\hline R Hya (41) & -29.40 & 1862. & 226. & 106. & 97. & 4.21 & 0.0040 & 2.37 \\
\hline R Hya (42) & -29.40 & 2069. & 251. & 111. & 110. & 1.42 & 0.0021 & 2.70 \\
\hline alpOri (46) & 11.68 & 182. & 33. & 33. & 700. & 7.00 & 0.0003 & 1.32 \\
\hline alpSco (55) & -32.16 & 558. & 108. & 58. & 746. & 3.39 & 0.0006 & 3.71 \\
\hline R Leo (60) & 9.55 & 970. & 132. & 76. & 448. & 7.00 & 0.0006 & 0.95 \\
\hline R Leo (61) & 9.55 & 962. & 131. & 76. & 420. & 7.00 & 0.0006 & 0.94 \\
\hline
\end{tabular}

${ }^{a}$ Cassini's mean distance from Saturn.

${ }^{b}$ Fresnel zone diameter $=\sqrt{2 \lambda D}$.

${ }^{c}$ Average unocculted stellar counts per integration, summed over 8 spectral channels at $2.92 \mu \mathrm{m}$.

${ }^{d}$ Projected radial velocity of the star in the A ring, at a radius of $125,000 \mathrm{~km}$. Values listed as $7.0 \mathrm{~km} \mathrm{~s}^{-1}$ are for shallow occultations and set to the default average.

${ }^{e}$ Predicted minimum detectable optical depth at $10 \mathrm{~km}$ resolution $(3-\sigma)$.

${ }^{f}$ Predicted maximum detectable optical depth at $10 \mathrm{~km}$ resolution $(3-\sigma)$. 


\begin{tabular}{|c|c|c|c|c|c|c|c|c|}
\hline Name (rev) & $\begin{array}{r}B_{*} \\
(\mathrm{deg})\end{array}$ & $\begin{array}{r}D^{a} \\
(\mathrm{Mm})\end{array}$ & $\begin{array}{r}D \theta_{*} \\
(\mathrm{~m})\end{array}$ & $\begin{array}{r}\text { F.Z. } \\
(\mathrm{m})\end{array}$ & $\begin{array}{r}F_{0}^{c} \\
(\mathrm{DN})\end{array}$ & $\begin{array}{c}\left|v_{\text {rad }}\right|^{d} \\
(\mathrm{~km} / \mathrm{s})\end{array}$ & $\tau_{\min }^{e}$ & $\tau_{\max }^{f}$ \\
\hline alpTrA (63) & -74.18 & 554. & 32. & 58. & 235 . & 7.00 & 0.0087 & 4.57 \\
\hline R Leo (63) & 9.55 & 1018. & 138. & 78. & 360. & 8.68 & 0.0008 & 0.90 \\
\hline R Cas (65) & 56.04 & 264. & 32. & 40. & 70. & 14.61 & 0.0175 & 3.21 \\
\hline alpCen (66) & -67.30 & 783. & 32. & 69 . & 290 . & 4.90 & 0.0050 & 4.87 \\
\hline R Leo (68) & 9.55 & 1027. & 139. & 79 . & 50 & 11.04 & 0.0060 & 0.55 \\
\hline CW Leo (70) & 11.38 & 1006. & 254 . & 78. & 350. & 8.61 & 0.0015 & 0.97 \\
\hline gamCru (71) & -62.35 & 599. & 71. & 60. & 491. & 6.92 & 0.0028 & 5.18 \\
\hline CW Leo (71) & 11.38 & 990. & 250 . & 77. & 180. & 7.00 & 0.0023 & 0.88 \\
\hline gamCru (72) & -62.35 & 617. & 73. & 61. & 645. & 6.94 & 0.0022 & 5.42 \\
\hline gamCru (73) & -62.35 & 596. & 70. & 60. & 624. & 6.95 & 0.0023 & 5.39 \\
\hline CW Leo (74) & 11.38 & 1012. & 255 . & 78. & 140. & 6.91 & 0.0021 & 0.91 \\
\hline R Leo (75) & 9.55 & 1042. & 142. & 79 . & 268. & 10.19 & 0.0011 & 0.84 \\
\hline gamCru (77) & -62.35 & 583. & 69. & 59 . & 105. & 7.03 & 0.0087 & 4.11 \\
\hline R Leo (77) & 9.55 & 1049. & 142. & 79 . & 285. & 9.19 & 0.0010 & 0.86 \\
\hline gamCru (78) & -62.35 & 584. & 69. & 59 . & 289. & 7.04 & 0.0033 & 5.01 \\
\hline etaCar (78) & -62.47 & 461. & 0 . & 53. & 64. & 3.98 & 0.0151 & 3.62 \\
\hline betGru (78) & -43.38 & 208. & 27 . & 35. & 272 . & 17.63 & 0.0043 & 3.53 \\
\hline gamCru (79) & -62.35 & 694. & 82 . & 65. & 697. & 6.63 & 0.0020 & 5.51 \\
\hline RS Cnc (80) & 29.96 & 825. & 60. & 70. & 305. & 7.14 & 0.0036 & 2.50 \\
\hline gamCru (81) & -62.35 & 685. & 81. & 64. & 571. & 6.64 & 0.0024 & 5.33 \\
\hline gamCru (82) & -62.35 & 690. & 82. & 64. & 720. & 6.63 & 0.0020 & 5.53 \\
\hline RS Cnc (85) & 29.96 & 830 . & 60. & 71. & 311. & 6.92 & 0.0035 & 2.51 \\
\hline gamCru (86) & -62.35 & 687. & 81. & 64. & 1031. & 6.65 & 0.0018 & 5.67 \\
\hline R Leo (86) & 9.55 & 1103. & 150. & 81. & 650. & 7.00 & 0.0006 & 0.96 \\
\hline RS Cnc (87) & 29.96 & 827. & 60. & 70 . & 324. & 6.83 & 0.0033 & 2.54 \\
\hline R Leo (87) & 9.55 & 1104. & 150. & 81. & 304. & 7.00 & 0.0008 & 0.89 \\
\hline gamCru (89) & -62.35 & 681. & 81. & 64. & 706 . & 6.68 & 0.0020 & 5.51 \\
\hline RS Cnc (92) & 29.96 & 767. & 56. & 68. & 224 . & 4.45 & 0.0033 & 2.53 \\
\hline gamCru (93) & -62.35 & 796. & 94. & 69. & 472. & 5.86 & 0.0023 & 5.34 \\
\hline gamCru (94) & -62.35 & 800. & 95. & 69. & 269. & 6.30 & 0.0033 & 5.00 \\
\hline
\end{tabular}




\begin{tabular}{|c|c|c|c|c|c|c|c|c|}
\hline Name (rev) & $\begin{array}{r}B_{*} \\
(\mathrm{deg})\end{array}$ & $\begin{array}{r}D^{a} \\
(\mathrm{Mm})\end{array}$ & $\begin{array}{r}D \theta_{*} \\
(\mathrm{~m})\end{array}$ & $\begin{array}{r}\text { F.Z. } \\
(\mathrm{m})\end{array}$ & $\begin{array}{r}F_{0}^{c} \\
(\mathrm{DN})\end{array}$ & $\begin{array}{c}\left|v_{\text {rad }}\right|^{d} \\
(\mathrm{~km} / \mathrm{s})\end{array}$ & $\tau_{\min }^{e}$ & $\tau_{\max }^{f}$ \\
\hline epsMus (94) & -72.77 & 670. & 42. & 63. & 190. & 3.57 & 0.0066 & 4.79 \\
\hline gamCru (96) & -62.35 & 752. & 89. & 67. & 200. & 6.90 & 0.0046 & 4.69 \\
\hline gamCru (97) & -62.35 & 751. & 89. & 67. & 858. & 6.90 & 0.0021 & 5.49 \\
\hline gamCru (100) & -62.35 & 886. & 105. & 73. & 384 . & 6.25 & 0.0026 & 5.21 \\
\hline $\operatorname{alp} \operatorname{Tr} \mathrm{A}(100)$ & -74.18 & 702. & 41. & 65. & 23. & 5.12 & 0.0734 & 2.48 \\
\hline gamCru (101) & -62.35 & 885. & 105. & 73. & 406. & 6.25 & 0.0028 & 5.18 \\
\hline gamCru (102) & -62.35 & 883. & 104. & 73. & 990. & 6.26 & 0.0018 & 5.66 \\
\hline TX Cam (102) & 61.29 & 810. & 22. & 70 . & 51. & 5.26 & 0.0216 & 3.26 \\
\hline gamCru (104) & -62.35 & 1094. & 129. & 81. & 226. & 0.57 & 0.0012 & 5.90 \\
\hline betPeg (104) & 31.68 & 825. & 60. & 70. & 295. & 11.72 & 0.0035 & 2.66 \\
\hline alpCen (105) & -67.30 & 1055. & 42. & 80 . & 190. & 5.34 & 0.0063 & 4.63 \\
\hline gamCru (106) & -62.35 & 1092. & 129. & 81. & 774 . & 0.64 & 0.0007 & 6.45 \\
\hline R Cas (106) & 56.04 & 856. & 104. & 72 . & 127. & 6.06 & 0.0063 & 4.07 \\
\hline betPeg (108) & 31.68 & 1207. & 88. & 85. & 290. & 9.33 & 0.0032 & 2.71 \\
\hline alpAur (110) & 50.88 & 909. & 23. & 74 . & 286. & 4.96 & 0.0049 & 3.97 \\
\hline alpAur (112) & 50.88 & 588. & 15. & 59 . & 393. & 7.00 & 0.0043 & 4.08 \\
\hline alpSco (115) & -32.16 & 982. & 190. & 77 . & 2703. & 3.36 & 0.0004 & 4.02 \\
\hline alpOri (117) & 11.68 & 277 . & 50. & 41. & 462. & 17.57 & 0.0008 & 1.15 \\
\hline omiCet (132) & 3.45 & 175. & 25. & 32 . & 400. & 37.75 & 0.0008 & 0.27 \\
\hline omiCet (135) & 3.45 & 350 . & 49. & 46. & 326. & 16.57 & 0.0005 & 0.29 \\
\hline alpCMa (168) & -13.48 & 307. & 8. & 43. & 39. & 7.00 & 0.0061 & 0.85 \\
\hline alpCMa (169) & -13.48 & 312 . & 8. & 43. & 38. & 7.00 & 0.0062 & 0.85 \\
\hline betPeg (170) & 31.68 & 926. & 67. & 75 . & 280. & 4.59 & 0.0023 & 2.88 \\
\hline betPeg (172) & 31.68 & 1131. & 82. & 82. & 278. & 4.59 & 0.0023 & 2.88 \\
\hline lamVel (173) & -43.81 & 478. & 29. & 54 . & 250. & 9.59 & 0.0060 & 3.32 \\
\hline alpCet (174) & 10.53 & 709. & 40. & 65. & 250. & 21.38 & 0.0027 & 0.78 \\
\hline alpLyr (175) & 35.22 & 2025 . & 32 . & 110. & 64. & 3.11 & 0.0124 & 2.22 \\
\hline R Lyr (176) & 40.77 & 1654. & 115. & 100. & 230. & 1.94 & 0.0022 & 3.74 \\
\hline W Hya (179) & -34.64 & 693. & 135. & 65. & 250. & 9.18 & 0.0028 & 3.06 \\
\hline R Lyr (180) & 40.77 & 1658. & 115 . & 100. & 230. & 1.82 & 0.0022 & 3.76 \\
\hline
\end{tabular}




\begin{tabular}{|c|c|c|c|c|c|c|c|c|}
\hline Name (rev) & $\begin{array}{r}B_{*} \\
(\operatorname{deg})\end{array}$ & $\begin{array}{r}D^{a} \\
(\mathrm{Mm})\end{array}$ & $\begin{array}{l}D \theta_{*} \\
(\mathrm{~m})\end{array}$ & $\begin{array}{r}\text { F.Z. }^{b} \\
(\mathrm{~m})\end{array}$ & $\begin{array}{r}F_{0}^{c} \\
(\mathrm{DN})\end{array}$ & $\begin{array}{c}\left|v_{\mathrm{rad}}\right|^{d} \\
(\mathrm{~km} / \mathrm{s})\end{array}$ & $\tau_{\min }^{e}$ & $\tau_{\text {max }}^{f}$ \\
\hline R Cas (180) & 56.04 & 978. & 119 . & 77. & 110. & 7.00 & 0.0110 & 3.60 \\
\hline W Hya (180) & -34.64 & 682. & 132. & 64. & 235 . & 9.16 & 0.0029 & 3.02 \\
\hline W Hya (181) & -34.64 & 690. & 134. & 64. & 265. & 9.17 & 0.0026 & 3.09 \\
\hline mu Cep (185) & 59.90 & 1104. & 75. & 81. & 200. & 3.81 & 0.0048 & 4.54 \\
\hline R Cas (185) & 56.04 & 973. & 118. & 76. & 75. & 5.16 & 0.0138 & 3.41 \\
\hline R Hya (185) & -29.40 & 823. & 100. & 70. & 250. & 6.79 & 0.0029 & 2.54 \\
\hline R Dor (186) & -56.27 & 391. & 108. & 48. & 600. & 5.22 & 0.0013 & 5.44 \\
\hline W Hya (186) & -34.64 & 813. & 158. & 70. & 245. & 9.15 & 0.0028 & 3.05 \\
\hline gamCru (187) & -62.35 & 548. & 65. & 57. & 320. & 7.58 & 0.0031 & 5.07 \\
\hline R Dor (188) & -56.27 & 399 . & 110. & 49. & 520. & 5.28 & 0.0015 & 5.31 \\
\hline W Hya (189) & -34.64 & 813. & 158. & 70. & 280. & 9.17 & 0.0025 & 3.12 \\
\hline mu Cep (191) & 59.90 & 1205. & 82. & 85. & 220 . & 4.82 & 0.0049 & 4.52 \\
\hline R Cas (191) & 56.04 & 1050. & 127. & 79. & 100. & 5.77 & 0.0110 & 3.60 \\
\hline R Car (191) & -63.48 & 654 & 63. & 63. & 85. & 7.43 & 0.0158 & 3.63 \\
\hline R Cas (192) & 56.04 & 1062. & 129. & 80. & 55. & 5.77 & 0.0198 & 3.11 \\
\hline mu Cep (193) & 59.90 & 1206. & 82. & 85. & 240. & 4.82 & 0.0045 & 4.59 \\
\hline mu Cep (194) & 59.90 & 1215. & 83. & 85. & 105. & 4.82 & 0.0070 & 4.18 \\
\hline R Cas (194) & 56.04 & 973. & 118. & 76. & 105. & 5.76 & 0.0105 & 3.64 \\
\hline etaCar (194) & -62.47 & 675. & 0 . & 64. & 115 . & 7.49 & 0.0167 & 3.55 \\
\hline 2 Cen (194) & -40.73 & 858. & 61. & 72. & 345. & 7.59 & 0.0043 & 3.32 \\
\hline mu Cep (195) & 59.90 & 1513. & 103. & 95. & 225. & 2.65 & 0.0035 & 4.79 \\
\hline W Hya (196) & -34.64 & 1116. & 216. & 82. & 340. & 5.99 & 0.0017 & 3.35 \\
\hline betAnd (196) & 41.52 & 2086 . & 123. & 112. & 460. & 7.00 & 0.0036 & 3.51 \\
\hline W Hya (197) & -34.64 & 1115. & 216. & 82. & 310 . & 5.89 & 0.0018 & 3.31 \\
\hline L2 Pup (198) & -41.91 & 1776. & 0 . & 103. & 210. & 7.00 & 0.0068 & 3.10 \\
\hline R Lyr (198) & 40.77 & 1396. & 97. & 92. & 235 . & 6.62 & 0.0041 & 3.35 \\
\hline L2 Pup (199) & -41.91 & 2493. & 0 . & 122. & 100. & 2.93 & 0.0063 & 3.13 \\
\hline R Lyr (199i) & 40.77 & 1239. & 86. & 86. & 215. & 6.34 & 0.0043 & 3.31 \\
\hline R Lyr (199e) & 40.77 & 1239. & 86. & 86. & 215. & 6.34 & 0.0043 & 3.31 \\
\hline R Lyr (200) & 40.77 & 1101. & 76. & 81. & 220 . & 7.77 & 0.0047 & 3.25 \\
\hline
\end{tabular}




\begin{tabular}{|c|c|c|c|c|c|c|c|c|}
\hline Name (rev) & $\begin{array}{r}B_{*} \\
(\mathrm{deg})\end{array}$ & $\begin{array}{r}D^{a} \\
(\mathrm{Mm})\end{array}$ & $\begin{array}{l}D \theta_{*} \\
(\mathrm{~m})\end{array}$ & $\begin{array}{r}\text { F.Z. } \\
(\mathrm{m})\end{array}$ & $\begin{array}{r}F_{0}^{c} \\
(\mathrm{DN})\end{array}$ & $\begin{array}{c}\left|v_{\mathrm{rad}}\right|^{d} \\
(\mathrm{~km} / \mathrm{s})\end{array}$ & $\tau_{\min }^{e}$ & $\tau_{\max }^{f}$ \\
\hline L2 Pup (201a) & -41.91 & 2713. & 0. & 128. & 130. & 1.37 & 0.0033 & 3.56 \\
\hline gamEri (201) & -7.39 & 2038. & 84. & 111. & 85. & 6.23 & 0.0021 & 0.53 \\
\hline L2 Pup (201b) & -41.91 & 2893. & 0. & 132. & 115. & 2.86 & 0.0054 & 3.23 \\
\hline alpLyr (202i) & 35.22 & 877. & 14. & 73. & 36. & 9.64 & 0.0386 & 1.56 \\
\hline R Lyr (202i) & 40.77 & 880. & 61. & 73. & 185. & 8.29 & 0.0057 & 3.12 \\
\hline alpLyr (202e) & 35.22 & 877. & 14. & 73. & 36. & 9.63 & 0.0386 & 1.56 \\
\hline R Lyr (202e) & 40.77 & 880 . & 61. & 73. & 185. & 8.29 & 0.0057 & 3.12 \\
\hline lamVel (203) & -43.81 & 3014. & 183. & 135. & 170. & 1.36 & 0.0030 & 3.80 \\
\hline L2 Pup (205i) & -41.91 & 2380 . & 0 . & 120. & 130. & 2.78 & 0.0048 & 3.32 \\
\hline L2 Pup (205e) & -41.91 & 2380 . & 0 . & 120. & 130. & 2.78 & 0.0048 & 3.32 \\
\hline alpLyr (206) & 35.22 & 1050. & 17. & 79. & 47. & 8.01 & 0.0233 & 1.86 \\
\hline R Lyr (206) & 40.77 & 1004. & 70. & 78. & 100. & 8.00 & 0.0072 & 2.96 \\
\hline L2 Pup (206) & -41.91 & 2277 . & 0 . & 117. & 80. & 3.65 & 0.0088 & 2.90 \\
\hline R Lyr (208) & 40.77 & 859. & 60. & 72. & 200. & 7.81 & 0.0051 & 3.19 \\
\hline alpLyr (209) & 35.22 & 716. & 11. & 66. & 65. & 7.00 & 0.0183 & 2.00 \\
\hline alpHer (211) & 9.27 & 572. & 94. & 59. & 360. & 7.09 & 0.0005 & 0.95 \\
\hline alpHer (212) & 9.27 & 456. & 75. & 52. & 375 . & 23.82 & 0.0009 & 0.85 \\
\hline X Oph (213) & 5.47 & 394. & 25. & 49. & 35. & 16.27 & 0.0042 & 0.30 \\
\hline 30 Psc (222) & -1.06 & 175. & 6. & 32. & 20. & 7.00 & 0.0009 & 0.06 \\
\hline omiCet (231) & 3.45 & 260. & 37. & 40. & 128. & 9.06 & 0.0006 & 0.28 \\
\hline omiCet (231) & 3.45 & 390. & 55. & 48. & 128. & 9.66 & 0.0006 & 0.28 \\
\hline R Aql (233) & 5.56 & 407. & 21. & 49. & 52. & 28.02 & 0.0054 & 0.28 \\
\hline epsPeg (233) & 11.53 & 384 . & 14. & 48. & 80. & 21.19 & 0.0063 & 0.69 \\
\hline W Hya (236) & -34.64 & 2845. & 552. & 131. & 750. & 1.84 & 0.0008 & 3.82 \\
\hline 2 Cen (237) & -40.73 & 2273. & 162. & 117. & 250 . & 1.66 & 0.0024 & 3.70 \\
\hline alpSco (237) & -32.16 & 1541. & 299. & 96. & 2300 . & 2.38 & 0.0003 & 4.11 \\
\hline betPeg (237) & 31.68 & 599. & 44. & 60. & 270. & 4.27 & 0.0023 & 2.88 \\
\hline alpSco (238) & -32.16 & 1549. & 301. & 96. & 1700. & 2.28 & 0.0004 & 3.96 \\
\hline alpSco (239) & -32.16 & 1065. & 207. & 80. & 580. & 7.65 & 0.0011 & 3.36 \\
\hline R Cas (239) & 56.04 & 588. & 71. & 59. & 100. & 5.51 & 0.0107 & 3.62 \\
\hline
\end{tabular}




\begin{tabular}{|c|c|c|c|c|c|c|c|c|}
\hline Name (rev) & $\begin{array}{r}B_{*} \\
(\mathrm{deg})\end{array}$ & $\begin{array}{r}D^{a} \\
(\mathrm{Mm})\end{array}$ & $\begin{array}{l}D \theta_{*} \\
(\mathrm{~m})\end{array}$ & $\begin{array}{r}\text { F.Z. } \\
(\mathrm{m})\end{array}$ & $\begin{array}{r}F_{0}^{c} \\
(\mathrm{DN})\end{array}$ & $\begin{array}{c}\left|v_{\text {rad }}\right|^{d} \\
(\mathrm{~km} / \mathrm{s})\end{array}$ & $\tau_{\text {min }}^{e}$ & $\tau_{\max }^{f}$ \\
\hline rhoPer (239) & 45.27 & 712 & 52. & 65. & 95. & 9.17 & 0.0125 & 2.88 \\
\hline alpOri (240) & 11.68 & 1030. & 187. & 79. & 950. & 10.49 & 0.0003 & 1.35 \\
\hline $\operatorname{alpSco}(241)$ & -32.16 & 702. & 136. & 65. & 660. & 9.27 & 0.0011 & 3.38 \\
\hline alpSco (241) & -32.16 & 645. & 125. & 62. & 680. & 9.26 & 0.0010 & 3.39 \\
\hline X Oph (241) & 5.47 & 495 . & 32. & 55. & 33. & 69.85 & 0.0093 & 0.22 \\
\hline alpOri (241) & 11.68 & 1032. & 188. & 79 . & 890. & 10.71 & 0.0003 & 1.33 \\
\hline alpSco (243) & -32.16 & 657. & 127. & 63. & 690. & 9.33 & 0.0010 & 3.40 \\
\hline X Oph (243) & 5.47 & 489. & 31. & 54. & 24 . & 70.22 & 0.0127 & 0.19 \\
\hline R Cas (243) & 56.04 & 476 . & 58. & 53. & 68. & 9.78 & 0.0209 & 3.06 \\
\hline lamVel (245) & -43.81 & 1239. & 75. & 86. & 44. & 7.00 & 0.0344 & 2.08 \\
\hline gamCru (245) & -62.35 & 743. & 88. & 67. & 290. & 4.35 & 0.0026 & 5.23 \\
\hline alpSco (245) & -32.16 & 442. & 86. & 52. & 700. & 12.58 & 0.0012 & 3.33 \\
\hline alpOri (245) & 11.68 & 1025. & 186. & 78. & 920. & 5.20 & 0.0002 & 1.41 \\
\hline lamVel (246) & -43.81 & 1240. & 75 & 86. & 275. & 7.00 & 0.0054 & 3.39 \\
\hline alpCen (247) & -67.30 & 643. & 26. & 62. & 256. & 7.00 & 0.0077 & 4.46 \\
\hline alpOri (247) & 11.68 & 1023. & 186. & 78. & 870. & 6.12 & 0.0003 & 1.38 \\
\hline etaCar (250) & -62.47 & 600. & 0 . & 60. & 55. & 4.60 & 0.0270 & 3.11 \\
\hline alpOri (253) & 11.68 & 968. & 176. & 76. & 930. & 4.88 & 0.0002 & 1.42 \\
\hline gamCru (255) & -62.35 & 430. & 51. & 51. & 250. & 8.98 & 0.0042 & 4.77 \\
\hline VY CMa (256) & -23.43 & 1130. & 102. & 82. & 85. & 2.61 & 0.0059 & 1.68 \\
\hline alpOri (256) & 11.68 & 971. & 177. & 76. & 900. & 7.23 & 0.0003 & 1.37 \\
\hline alpOri (260) & 11.68 & 970. & 176. & 76. & 900. & 9.43 & 0.0003 & 1.35 \\
\hline VY CMa (262) & -23.43 & 1131. & 103. & 82. & 160. & 4.22 & 0.0041 & 1.84 \\
\hline alpOri (262) & 11.68 & 962. & 175. & 76. & 700. & 10.34 & 0.0004 & 1.29 \\
\hline gamCru (264) & -62.35 & 452. & 53. & 52. & 270. & 9.11 & 0.0040 & 4.84 \\
\hline lamVel (265) & -43.81 & 760. & 46. & 68. & 20. & 5.96 & 0.0655 & 1.64 \\
\hline lamVel (268) & -43.81 & 770 . & 47. & 68. & 200. & 5.95 & 0.0068 & 3.23 \\
\hline gamCru (268) & -62.35 & 387. & 46. & 48. & 320. & 7.00 & 0.0030 & 5.10 \\
\hline alpOri (268) & 11.68 & 985. & 179. & 77. & 900. & 12.16 & 0.0004 & 1.32 \\
\hline VY CMa (269) & -23.43 & 1155. & 105. & 83. & 220 . & 5.31 & 0.0034 & 1.92 \\
\hline
\end{tabular}




\begin{tabular}{|l|r|r|r|r|r|r|r|r|}
\hline Name (rev) & $\begin{array}{r}B_{*} \\
(\mathrm{deg})\end{array}$ & $\begin{array}{r}D^{a} \\
(\mathrm{Mm})\end{array}$ & $\begin{array}{r}D \theta_{*} \\
(\mathrm{~m})\end{array}$ & $\begin{array}{r}\mathrm{F}^{\prime} .^{b} \\
(\mathrm{~m})\end{array}$ & $\begin{array}{r}F_{0}^{c} \\
(\mathrm{DN})\end{array}$ & $\begin{array}{r}\left|v_{\text {rad }}\right|^{d} \\
(\mathrm{~km} / \mathrm{s})\end{array}$ & $\tau_{\min }^{e}$ & $\tau_{\max }^{f}$ \\
\hline etaCar (269) & -62.47 & 520. & 0. & 56. & 117. & 7.45 & 0.0163 & 3.56 \\
gamCru (269) & -62.35 & 428. & 51. & 51. & 320. & 9.17 & 0.0034 & 4.98 \\
alpOri (269) & 11.68 & 985. & 179. & 77. & 900. & 12.38 & 0.0004 & 1.32 \\
alpCMa (272) & -13.48 & 1133. & 31. & 82. & 75. & 4.23 & 0.0035 & 0.98 \\
alpCMa (274) & -13.48 & 1106. & 30. & 81. & 125. & 6.20 & 0.0026 & 1.06 \\
& & & & & & & & \\
gamCru (276) & -62.35 & 242. & 29. & 38. & 215. & 13.42 & 0.0060 & 4.46 \\
alpOri (277) & 11.68 & 755. & 137. & 67. & 970. & 10.12 & 0.0003 & 1.35 \\
alpCMa (281) & -13.48 & 1152. & 31. & 83. & 120. & 5.68 & 0.0026 & 1.06 \\
alpCMa (282) & -13.48 & 1153. & 31. & 83. & 120. & 4.68 & 0.0023 & 1.08 \\
gamCru (282) & -62.35 & 230. & 27. & 37. & 260. & 13.89 & 0.0051 & 4.61 \\
gamCru (291) & -62.35 & 232. & 27. & 37. & 260. & 14.81 & 0.0053 & 4.59 \\
gamCru (292) & -62.35 & 233. & 28. & 37. & 315. & 14.90 & 0.0044 & 4.75 \\
\hline
\end{tabular}


Table 11: Geometric data for ring chord occultations.

\begin{tabular}{|c|c|c|c|c|}
\hline Name $(\text { rev })^{a}$ & $\begin{array}{r}B_{*} \\
(\mathrm{deg})\end{array}$ & $\begin{array}{l}R_{\min }^{b} \\
(\mathrm{~km})\end{array}$ & $\begin{array}{r}v_{\mathrm{rad}}^{c} \\
(\mathrm{~km} / \mathrm{s})\end{array}$ & $\begin{array}{c}v_{\mathrm{rad}}^{d} \\
(\mathrm{~km} / \mathrm{s})\end{array}$ \\
\hline omiCet (8) & 3.45 & 114960. & -5.70 & 5.71 \\
\hline omiCet (9) & 3.45 & 125920 . & & \\
\hline omiCet (10) & 3.45 & 132015. & & \\
\hline omiCet (12) & 3.45 & 139490. & & \\
\hline alpSco (13) & -32.16 & 101172. & -10.07 & 10.28 \\
\hline delVir (29) & -2.38 & 70618. & -180.89 & 181.58 \\
\hline R Leo (30) & 9.55 & 109537. & -26.56 & 26.72 \\
\hline CW Leo (31) & 11.38 & 91362 . & -40.16 & 40.27 \\
\hline R Leo (60) & 9.55 & 126095. & & \\
\hline R Leo (61) & 9.55 & 127918. & & \\
\hline $\operatorname{alp} \operatorname{Tr} \mathrm{A}(63)$ & -74.18 & 127179. & & \\
\hline R Leo (63) & 9.55 & 114770. & -8.66 & 8.68 \\
\hline R Leo (68) & 9.55 & 104095. & -11.03 & 11.04 \\
\hline CW Leo (70) & 11.38 & 105664. & -8.60 & 8.61 \\
\hline CW Leo (74) & 11.38 & 112470. & -7.02 & 6.91 \\
\hline R Leo (75) & 9.55 & 104177. & -10.20 & 10.19 \\
\hline R Leo (77) & 9.55 & 108281. & -9.18 & 9.19 \\
\hline RS Cnc (80) & 29.96 & 78000. & -7.13 & 7.14 \\
\hline RS Cnc (85) & 29.96 & 81116. & -6.91 & 6.92 \\
\hline R Leo (86) & 9.55 & 127256. & & \\
\hline
\end{tabular}

${ }^{a}$ Star name (orbit number).

${ }^{b}$ Minimum stellar radius from Saturn, projected into the ring plane.

${ }^{c}$ Projected radial velocity of the star in the ring plane, at a radius of $125,000 \mathrm{~km}$ (ingress). A blank indicates that the occultation track did not penetrate as far in as, or began interior to, this radius.

${ }^{d}$ Projected radial velocity of the star in the ring plane, at a radius of $125,000 \mathrm{~km}$ (egress). A blank indicates that the occultation track did not penetrate as far in as, or ended interior to, this radius. 


\begin{tabular}{|c|c|c|c|c|}
\hline Name $(\mathrm{rev})^{a}$ & $\begin{array}{r}B_{*} \\
(\mathrm{deg})\end{array}$ & $\begin{array}{l}R_{\text {min }}^{b} \\
(\mathrm{~km})\end{array}$ & $\begin{array}{r}v_{\mathrm{rad}}^{c} \\
(\mathrm{~km} / \mathrm{s})\end{array}$ & $\begin{array}{c}v_{\mathrm{rad}}^{d} \\
(\mathrm{~km} / \mathrm{s})\end{array}$ \\
\hline RS Cnc (87) & 29.96 & 82502 . & -6.82 & 6.83 \\
\hline R Leo (87) & 9.55 & 128136. & & \\
\hline RS Cnc (92) & 29.96 & 111362. & -4.45 & \\
\hline epsMus (94) & -72.77 & 104886. & -3.59 & 3.57 \\
\hline alpTrA (100) & -74.18 & 91657. & -5.13 & 5.12 \\
\hline gamCru (104) & -62.35 & 124413. & -0.57 & 0.57 \\
\hline gamCru (106) & -62.35 & 124263. & -0.64 & 0.64 \\
\hline alpAur (110) & 50.88 & 95401. & -4.96 & 4.96 \\
\hline omiCet (132) & 3.45 & 110303. & -37.62 & 37.75 \\
\hline alpCet (174) & 10.53 & 79855. & -21.38 & \\
\hline R Lyr (176) & 40.77 & 106447. & -1.94 & 1.94 \\
\hline R Lyr (180) & 40.77 & 108785. & -1.82 & 1.82 \\
\hline R Cas (180) & 56.04 & 139029. & & \\
\hline R Hya (185) & -29.40 & 95301. & -6.78 & 6.79 \\
\hline R Dor (186) & -56.27 & 115196. & -5.22 & \\
\hline gamCru (187) & -62.35 & 65072. & -7.51 & 7.58 \\
\hline R Dor (188) & -56.27 & 114972. & -5.27 & 5.28 \\
\hline 2 Cen (194) & -40.73 & 64514. & -7.59 & 7.59 \\
\hline W Hya (196) & -34.64 & 98396. & -5.99 & 5.99 \\
\hline betAnd (196) & 41.52 & 133711. & & \\
\hline W Hya (197) & -34.64 & 99425. & -5.89 & \\
\hline L2 Pup (198) & -41.91 & 131490. & & \\
\hline L2 Pup (201) & -41.91 & 113069. & -1.37 & 1.37 \\
\hline gamEri (201) & -7.39 & 119306. & -6.23 & 6.23 \\
\hline lamVel (203) & -43.81 & 102475. & -1.36 & 1.36 \\
\hline
\end{tabular}




\begin{tabular}{|l|r|r|r|r|}
\hline Name (rev) & $B_{*}$ & $\begin{array}{r}R_{\mathrm{min}}^{b} \\
(\mathrm{~km})\end{array}$ & $\begin{array}{r}v_{\text {rad }}^{c} \\
(\mathrm{~km} / \mathrm{s})\end{array}$ & $\begin{array}{r}v_{\text {rad }}^{d} \\
(\mathrm{~km} / \mathrm{s})\end{array}$ \\
\hline alpHer (211) & 9.27 & 121006. & -7.09 & 7.09 \\
30 Psc (222) & -1.06 & 128385. & & \\
R Aql (233) & 5.56 & 91791. & -33.75 & 28.02 \\
2 Cen (237) & -40.73 & 100072. & -1.66 & 1.66 \\
alpSco (237) & -32.16 & 112115. & -2.38 & 2.38 \\
& & & & \\
betPeg (237) & 31.68 & 115201. & -4.26 & 4.27 \\
alpSco (238) & -32.16 & 113211. & -2.28 & 2.28 \\
R Cas (239) & 56.04 & 103515. & -5.50 & 5.51 \\
alpOri (240) & 11.68 & 105888. & -10.50 & 10.49 \\
X Oph (241) & 5.47 & 79783. & -69.87 & 69.85 \\
& & & & \\
alpOri (241) & 11.68 & 105026. & -10.71 & 10.71 \\
X Oph (243) & 5.47 & 78826. & -70.43 & 70.22 \\
lamVel (245) & -43.81 & 126181. & & \\
gamCru (245) & -62.35 & 78796. & & 4.35 \\
alpSco (245) & -32.16 & 70325. & -12.63 & 12.58 \\
& & & & \\
alpOri (245) & 11.68 & 119828. & -5.19 & 5.20 \\
lamVel (246) & -43.81 & 125193. & & \\
alpCen (247) & -67.30 & 139000. & & \\
alpOri (247) & 11.68 & 117737. & -6.12 & 6.12 \\
etaCar (250) & -62.47 & 83617. & -4.76 & 4.60 \\
alpOri (253) & 11.68 & 117434. & -4.87 & 4.88 \\
VY CMa (256) & -23.43 & 114246. & -2.61 & 2.61 \\
alpOri (256) & 11.68 & 107675. & -7.23 & 7.23 \\
alpOri (260) & 11.68 & 93813. & -9.44 & 9.43 \\
VY CMa (262) & -23.43 & 94017. & -4.22 & 4.22 \\
alpOri (262) & 11.68 & 85856. & -10.34 & 10.34 \\
alpCMa (272) & -13.48 & 101028. & -4.24 & 4.23 \\
alpCMa (281) & -13.48 & 72323. & -5.68 & 5.68 \\
alpCMa (282) & -13.48 & 92210. & -4.68 & 4.68 \\
\hline
\end{tabular}

